\title{
Commissioning of the ATLAS Muon Spectrometer with cosmic rays
}

\author{
The ATLAS Collaboration ${ }^{\star \star \star}$
}

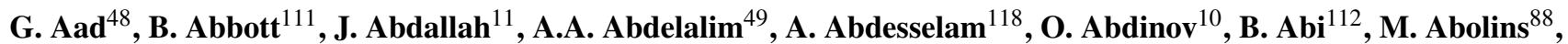
H. Abramowicz ${ }^{152}$, H. Abreu ${ }^{15}$, B.S. Acharya ${ }^{163 a, 163 b}$, D.L. Adams ${ }^{24}$, T.N. Addy ${ }^{56}$, J. Adelman ${ }^{174}$, C. Adorisio $^{36 a, 36 b}$, P. Adragna ${ }^{75}$, T. Adye ${ }^{129}$, S. Aefsky ${ }^{22}$, J.A. Aguilar-Saavedra124b ${ }^{12}$ M. Aharrouche ${ }^{81}$, S.P. Ahlen ${ }^{21}$, F. Ahles ${ }^{48}$, A. Ahmad ${ }^{147}$, H. Ahmed ${ }^{2}$, M. Ahsan ${ }^{40}$, G. Aielli ${ }^{133 a, 133 b}$, T. Akdogan ${ }^{18 a}$, T.P.A. Åkesson ${ }^{79}$, G. Akimoto $^{154}$, A.V. Akimov ${ }^{94}$, A. Aktas $^{48}$, M.S. Alam ${ }^{1}$, M.A. Alam ${ }^{76}$, S. Albrand ${ }^{55}$, M. Aleksa ${ }^{29}$, I.N. Aleksandrov ${ }^{65}$, C. Alexa ${ }^{25 a}$, G. Alexander ${ }^{152}$, G. Alexandre ${ }^{49}$, T. Alexopoulos ${ }^{9}$, M. Alhroob ${ }^{20}$, M. Aliev ${ }^{15}$, G. Alimonti ${ }^{89}$ a , J. Alison ${ }^{120}$, M. Aliyev ${ }^{10}$, P.P. Allport ${ }^{73}$, S.E. Allwood-Spiers ${ }^{53}$, J. Almond ${ }^{82}$, A. Aloisio ${ }^{102 a}, 102 b$, R. Alon ${ }^{170}$, A. Alonso $^{79}$, M.G. Alviggi ${ }^{102 a, 102 b}$, K. Amako ${ }^{66}$, C. Amelung ${ }^{22}$, A. Amorim ${ }^{124 a}$, G. Amorós ${ }^{166}$, N. Amram ${ }^{152}$, C. Anastopoulos $^{139}$, T. Andeen ${ }^{29}$, C.F. Anders ${ }^{48}$, K.J. Anderson ${ }^{30}$, A. Andreazza ${ }^{89 a}$,89b , V. Andrei $^{58 a}$, X.S. Anduaga ${ }^{70}$, A. Angerami $^{34}$, F. Anghinolfi ${ }^{29}$, N. Anjos ${ }^{124 a}$, A. Annovi ${ }^{47}$, A. Antonaki ${ }^{8}$, M. Antonelli ${ }^{47}$, S. Antonelli $^{19 a, 19 b}$, J. Antos ${ }^{144 b}$, B. Antunovic ${ }^{41}$, F. Anulli ${ }^{132 a}$, S. Aoun ${ }^{83}$, G. Arabidze ${ }^{8}$, I. Aracena ${ }^{143}$, Y. Arai ${ }^{66}$, A.T.H. Arce ${ }^{14}$, J.P. Archambault ${ }^{28}$, S. Arfaoui ${ }^{29, a}$, J.-F. Arguin ${ }^{14}$, T. Argyropoulos ${ }^{9}$, M. Arik ${ }^{18 a}$, A.J. Armbruster ${ }^{87}$, O. Arnaez ${ }^{4}$, C. Arnault ${ }^{115}$, A. Artamonov ${ }^{95}$, D. Arutinov ${ }^{20}$, M. Asai ${ }^{143}$, S. Asai ${ }^{154}$, R. Asfandiyarov ${ }^{171}$, S. Ask ${ }^{82}$, B. Åsman ${ }^{145 a, 145 b}$, D. Asner ${ }^{28}$, L. Asquith ${ }^{77}$, K. Assamagan ${ }^{24}$, A. Astbury ${ }^{168}$, A. Astvatsatourov ${ }^{52}$, G. Atoian ${ }^{174}$, B. Auerbach ${ }^{174}$, K. Augsten $^{127}$, M. Aurousseau ${ }^{4}$, N. Austin ${ }^{73}$, G. Avolio ${ }^{162}$, R. Avramidou ${ }^{9}$, D. Axen ${ }^{167}$, C. Ay ${ }^{54}$, G. Azuelos ${ }^{93, b}$, Y. Azuma ${ }^{154}$, M.A. Baak ${ }^{29}$, A.M. Bach ${ }^{14}$, H. Bachacou ${ }^{136}$, K. Bachas ${ }^{29}$, M. Backes ${ }^{49}$, E. Badescu ${ }^{25 a}$, P. Bagnaia ${ }^{132 a, 132 b}$, Y. Bai ${ }^{32 a}$, T. Bain ${ }^{157}$, J.T. Baines ${ }^{129}$, O.K. Baker ${ }^{174}$, M.D. Baker ${ }^{24}$, S. Baker ${ }^{77}$, F. Baltasar Dos Santos Pedrosa ${ }^{29}$, E. Banas $^{38}$,

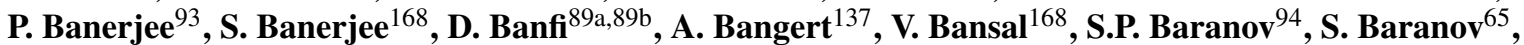
A. Barashkou ${ }^{65}$, T. Barber ${ }^{27}$, E.L. Barberio ${ }^{86}$, D. Barberis ${ }^{50 a, 50 b}$, M. Barbero ${ }^{20}$, D.Y. Bardin ${ }^{65}$, T. Barillari ${ }^{99}$, M. Barisonzi ${ }^{173}$, T. Barklow ${ }^{143}$, N. Barlow ${ }^{27}$, B.M. Barnett ${ }^{129}$, R.M. Barnett ${ }^{14}$, A. Baroncelli ${ }^{134 a}$, A.J. Barr ${ }^{118}$, F. Barreiro ${ }^{80}$, J. Barreiro Guimarães da Costa ${ }^{57}$, P. Barrillon ${ }^{115}$, R. Bartoldus ${ }^{143}$, D. Bartsch ${ }^{20}$, R.L. Bates $^{53}$, L. Batkova ${ }^{144}$, J.R. Batley ${ }^{27}$, A. Battaglia ${ }^{16}$, M. Battistin ${ }^{29}$, F. Bauer ${ }^{136}$, H.S. Bawa ${ }^{143}$, M. Bazalova ${ }^{125}$, B. Beare ${ }^{157}$, T. Beau ${ }^{78}$, P.H. Beauchemin ${ }^{118}$, R. Beccherle ${ }^{50 a}$, N. Becerici ${ }^{18 a}$, P. Bechtle ${ }^{41}$, G.A. Beck ${ }^{75}$, H.P. Beck $^{16}$, M. Beckingham ${ }^{48}$, K.H. Becks ${ }^{173}$, A.J. Beddall ${ }^{18 c}$, A. Beddall ${ }^{18 c}$, V.A. Bednyakov ${ }^{65}$, C. Bee $^{83}$, M. Begel $^{24}$, S. Behar Harpaz ${ }^{151}$, P.K. Behera ${ }^{63}$, M. Beimforde ${ }^{99}$, C. Belanger-Champagne ${ }^{165}$, P.J. Bell ${ }^{49}$, W.H. Bell ${ }^{49}$, G. Bella ${ }^{152}$, L. Bellagamba ${ }^{19 a}$, F. Bellina ${ }^{29}$, M. Bellomo ${ }^{119 a}$, A. Belloni ${ }^{57}$, K. Belotskiy ${ }^{96}$, O. Beltramello ${ }^{29}$, S. Ben Ami ${ }^{151}$, O. Benary ${ }^{152}$, D. Benchekroun ${ }^{135 a}$, M. Bendel ${ }^{81}$, B.H. Benedict ${ }^{162}$, N. Benekos ${ }^{164}$, Y. Benhammou ${ }^{152}$, G.P. Benincasa ${ }^{124 a}$, D.P. Benjamin ${ }^{44}$, M. Benoit ${ }^{115}$, J.R. Bensinger ${ }^{22}$, K. Benslama ${ }^{130}$, S. Bentvelsen ${ }^{105}$, M. Beretta $^{47}$, D. Berge ${ }^{29}$, E. Bergeaas Kuutmann ${ }^{41}$, N. Berger ${ }^{4}$, F. Berghaus ${ }^{168}$, E. Berglund ${ }^{49}$, J. Beringer ${ }^{14}$, P. Bernat ${ }^{115}$, R. Bernhard ${ }^{48}$, C. Bernius ${ }^{77}$, T. Berry ${ }^{76}$, A. Bertin ${ }^{19 a, 19 b}$, M.I. Besana ${ }^{89 a, 89 b}$, N. Besson ${ }^{136}$, S. Bethke ${ }^{99}$, R.M. Bianchi ${ }^{48}$, M. Bianco ${ }^{72 a, 72 b}$, O. Biebel ${ }^{98}$, J. Biesiada ${ }^{14}$, M. Biglietti ${ }^{132 a, 132 b}$, H. Bilokon ${ }^{47}$, M. Bindi $^{19 a, 19 b}$, S. Binet ${ }^{115}$, A. Bingul ${ }^{18 c}$, C. Bini ${ }^{132 a, 132 b}$, C. Biscarat ${ }^{179}$, U. Bitenc ${ }^{48}$, K.M. Black ${ }^{57}$, R.E. Blair ${ }^{5}$, J.-B. Blanchard ${ }^{115}$, G. Blanchot ${ }^{29}$, C. Blocker ${ }^{22}$, A. Blondel ${ }^{49}$, W. Blum ${ }^{81}$, U. Blumenschein ${ }^{54}$, G.J. Bobbink ${ }^{105}$, A. Bocci $^{44}$, M. Boehler ${ }^{41}$, J. Boek ${ }^{173}$, N. Boelaert ${ }^{79}$, S. Böser ${ }^{77}$, J.A. Bogaerts ${ }^{29}$, A. Bogouch ${ }^{90 \dagger}$, C. Bohm $^{145 a}$, J. Bohm $^{125}$, V. Boisvert ${ }^{76}$, T. Bold ${ }^{162, c}$, V. Boldea ${ }^{25 a}$, V.G. Bondarenko ${ }^{96}$, M. Bondioli ${ }^{162}$, M. Boonekamp ${ }^{136}$, S. Bordoni $^{78}$, C. Borer ${ }^{16}$, A. Borisov ${ }^{128}$, G. Borissov ${ }^{71}$, I. Borjanovic ${ }^{72 a}$, S. Borroni $^{132 a, 132 b}$, K. Bos ${ }^{105}$, D. Boscherini $^{19 a}$, M. Bosman ${ }^{11}$, H. Boterenbrood ${ }^{105}$, J. Bouchami ${ }^{93}$, J. Boudreau ${ }^{123}$, E.V. Bouhova-Thacker ${ }^{71}$, C. Boulahouache $^{123}$, C. Bourdarios ${ }^{115}$, A. Boveia ${ }^{30}$, J. Boyd ${ }^{29}$, I.R. Boyko ${ }^{65}$, I. Bozovic-Jelisavcic ${ }^{12 b}$, J. Bracinik ${ }^{17}$, A. Braem $^{29}$, P. Branchini ${ }^{134 a}$, G.W. Brandenburg ${ }^{57}$, A. Brandt ${ }^{7}$, G. Brandt ${ }^{41}$, O. Brandt ${ }^{54}$, U. Bratzler ${ }^{155}$, B. Brau ${ }^{84}$, J.E. Brau ${ }^{14}$, H.M. Braun ${ }^{173}$, B. Brelier ${ }^{157}$, J. Bremer ${ }^{29}$, R. Brenner ${ }^{165}$, S. Bressler ${ }^{151}$, D. Britton ${ }^{53}$, F.M. Brochu ${ }^{27}$, I. Brock ${ }^{20}$, R. Brock ${ }^{88}$, E. Brodet ${ }^{152}$, C. Bromberg ${ }^{88}$, G. Brooijmans ${ }^{34}$, W.K. Brooks ${ }^{31 b}$, G. Brown $^{82}$, P.A. Bruckman de Renstrom ${ }^{38}$, D. Bruncko ${ }^{144 b}$, R. Bruneliere ${ }^{48}{\text {, S. } \text { Brunet }^{41} \text {, A. Bruni }}^{19 a}$, G. Bruni $^{19 a}$, M. Bruschi ${ }^{19}$, F. Bucci ${ }^{49}$, J. Buchanan ${ }^{118}$, P. Buchholz ${ }^{141}$, A.G. Buckley ${ }^{45}$, I.A. Budagov ${ }^{65}$, B. Budick $^{108}$, 


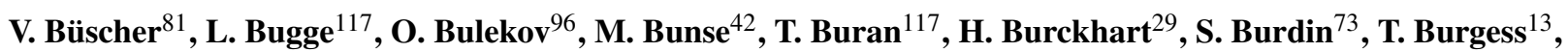
S. Burke ${ }^{129}$, E. Busato ${ }^{33}$, P. Bussey ${ }^{53}$, C.P. Buszello ${ }^{165}$, F. Butin ${ }^{29}$, B. Butler ${ }^{143}$, J.M. Butler ${ }^{21}$, C.M. Buttar ${ }^{53}$, J.M. Butterworth ${ }^{77}$, T. Byatt ${ }^{77}$, J. Caballero ${ }^{24}$, S. Cabrera Urbán ${ }^{166}$, D. Caforio ${ }^{19 a, 19 b}$, O. Cakir $^{3 a}$, P. Calafiura ${ }^{14}$, G. Calderini $^{78}$, P. Calfayan ${ }^{98}$, R. Calkins ${ }^{106 a}$, L.P. Caloba ${ }^{23 a}$, D. Calvet $^{33}$, P. Camarri ${ }^{133 a, 133 b}$, D. Cameron ${ }^{117}$, S. Campana ${ }^{29}$, M. Campanelli ${ }^{77}$, V. Canale ${ }^{102 a, 102 b}$, F. Canelli ${ }^{30}$, A. Canepa ${ }^{158 a}$, J. Cantero ${ }^{80}$, L. Capasso ${ }^{102 a, 102 b}$, M.D.M. Capeans Garrido ${ }^{29}$, I. Caprini ${ }^{25 a}$, M. Caprini ${ }^{25 a}$, M. Capua ${ }^{36 a, 36 b}$, R. Caputo ${ }^{147}$, C. Caramarcu ${ }^{25 a}$, R. Cardarelli ${ }^{133 a}$, T. Carli ${ }^{29}$, G. Carlino ${ }^{102 a}$, L. Carminati ${ }^{89 a, 89 b}$, B. Caron ${ }^{2, b}$, S. Caron ${ }^{48}$, G.D. Carrillo Montoya ${ }^{171}$, S. Carron Montero ${ }^{157}$, A.A. Carter $^{75}$, J.R. Carter $^{27}$, J. Carvalho ${ }^{124 a}$, D. Casadei ${ }^{108}$, M.P. Casado ${ }^{11}$,

M. Cascella ${ }^{122 a, 122 b}$, A.M. Castaneda Hernandez ${ }^{171}$, E. Castaneda-Miranda ${ }^{171}$, V. Castillo Gimenez ${ }^{166}$, N.F. Castro ${ }^{124 b}$, G. Cataldi ${ }^{72 a}$, A. Catinaccio ${ }^{29}$, J.R. Catmore $^{71}$, A. Cattai ${ }^{29}$, G. Cattani ${ }^{133 a, 133 b}$, S. Caughron ${ }^{34}$, D. Cauz $^{163 a, 163 c}$, P. Cavalleri ${ }^{78}$, D. Cavalli ${ }^{89 a}$, M. Cavalli-Sforza ${ }^{11}$, V. Cavasinni ${ }^{122 a, 122 b}$, F. Ceradini ${ }^{134 a, 134 b}$, A.S. Cerqueira ${ }^{23 a}$, A. Cerri $^{29}$, L. Cerrito ${ }^{75}$, F. Cerutti ${ }^{47}$, S.A. Cetin ${ }^{18 b}$, A. Chafaq ${ }^{135 a}$, D. Chakraborty ${ }^{106 a}$, K. Chan $^{2}$, J.D. Chapman ${ }^{27}$, J.W. Chapman ${ }^{87}$, E. Chareyre ${ }^{78}$, D.G. Charlton ${ }^{17}$, V. Chavda ${ }^{82}$, S. Cheatham ${ }^{71}$, S. Chekanov ${ }^{5}$, S.V. Chekulaev ${ }^{158 a}$, G.A. Chelkov ${ }^{65}$, H. Chen ${ }^{24}$, S. Chen ${ }^{32 c}$, X. Chen ${ }^{171}$, A. Cheplakov ${ }^{65}$, V.F. Chepurnov ${ }^{65}$, R. Cherkaoui El Moursli ${ }^{135 d}$, V. Tcherniatine ${ }^{24}$, D. Chesneanu ${ }^{25 a}$, E. Cheu ${ }^{6}$, S.L. Cheung ${ }^{157}$, L. Chevalier ${ }^{136}$, F. Chevallier ${ }^{136}$, V. Chiarella ${ }^{47}$, G. Chiefari ${ }^{102 a, 102 b}$, L. Chikovani ${ }^{51}$, J.T. Childers ${ }^{58 a}$, A. Chilingarov ${ }^{71}$, G. Chiodini $^{72 a}$, V. Chizhov ${ }^{65}$, G. Choudalakis ${ }^{30}$, S. Chouridou ${ }^{137}$, I.A. Christidi ${ }^{77}$, A. Christov ${ }^{48}$, D. Chromek-Burckhart ${ }^{29}$, M.L. Chu ${ }^{150}$, J. Chudoba ${ }^{125}$, G. Ciapetti ${ }^{132 a, 132 b}$, A.K. Ciftci $^{3 a}$, R. Ciftci ${ }^{3 a}$, D. Cinca ${ }^{33}$, V. Cindro ${ }^{74}$, M.D. Ciobotaru ${ }^{162}$, C. Ciocca ${ }^{19 a, 19 b}$, A. Ciocio ${ }^{14}$, M. Cirilli ${ }^{87}$, M. Citterio $^{89 a}$, A. Clark ${ }^{49}$, P.J. Clark $^{45}$, W. Cleland ${ }^{123}$, J.C. Clemens ${ }^{83}$, B. Clement ${ }^{55}$, C. Clement ${ }^{145 a, 145 b}$, Y. Coadou ${ }^{83}$, M. Cobal ${ }^{163 a, 163 c}$, A. Coccaro ${ }^{50 a, 50 b}$, J. Cochran ${ }^{64}$, J. Coggeshall ${ }^{164}$, E. Cogneras ${ }^{179}$, A.P. Colijn ${ }^{105}$, C. Collard ${ }^{115}$, N.J. Collins ${ }^{17}$, C. Collins-Tooth ${ }^{53}$, J. Collot ${ }^{55}$, G. Colon ${ }^{84}$, P. Conde Muiño ${ }^{124 a}$, E. Coniavitis ${ }^{165}$, M. Consonni ${ }^{104}$, S. Constantinescu ${ }^{25 a}$, C. Conta ${ }^{119 a, 119 b}$, F. Conventi ${ }^{102 a, d}$, M. Cooke ${ }^{34}$, B.D. Cooper ${ }^{75}$, A.M. Cooper-Sarkar ${ }^{118}$, N.J. Cooper-Smith ${ }^{76}$,

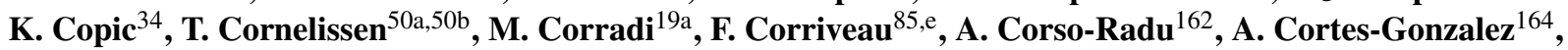
G. Cortiana $^{99}$, G. Costa ${ }^{89 a}$, M.J. Costa ${ }^{166}$, D. Costanzo ${ }^{139}$, T. Costin ${ }^{30}$, D. Côtée ${ }^{41}$, R. Coura Torres ${ }^{23 a}$, L. Courneyea ${ }^{168}$, G. Cowan $^{76}$, C. Cowden ${ }^{27}$, B.E. Cox $^{82}$, K. Cranmer ${ }^{108}$, J. Cranshaw ${ }^{5}$, M. Cristinziani ${ }^{20}$, G. Crosetti $^{36 a, 36 b}$, R. Crupi ${ }^{72 a, 72 b}$, S. Crépé-Renaudin ${ }^{55}$, C .Cuenca Almenar ${ }^{174}$, T. Cuhadar Donszelmann ${ }^{139}$, M. Curatolo ${ }^{47}$, C.J. Curtis ${ }^{17}$, P. Cwetanski ${ }^{61}$, Z. Czyczula ${ }^{174}$, S. D'Auria ${ }^{53}$, M. D'Onofrio ${ }^{73}$, A. D'Orazio ${ }^{99}$, C. Da Via ${ }^{82}$, W. Dabrowski ${ }^{37}$, T. Dai ${ }^{87}$, C. Dallapiccola ${ }^{84}$, S.J. Dallison ${ }^{129, *}$, C.H. Daly ${ }^{138}$, M. Dam ${ }^{35}$, H.O. Danielsson ${ }^{29}$, D. Dannheim ${ }^{99}$, V. Dao ${ }^{49}$, G. Darbo ${ }^{50 a}$, G.L. Darlea ${ }^{25 b}$, W. Davey ${ }^{86}$, T. Davidek ${ }^{126}$, N. Davidson ${ }^{86}$, R. Davidson ${ }^{71}$, M. Davies ${ }^{93}$, A.R. Davison ${ }^{77}$, I. Dawson ${ }^{139}$, R.K. Daya ${ }^{39}$, K. De $^{7}$, R. de Asmundis ${ }^{102 a}$, S. De Castro ${ }^{19 a, 19 b}$, P.E. De Castro Faria Salgado ${ }^{24}$, S. De Cecco ${ }^{78}$, J. de Graat ${ }^{98}$, N. De Groot ${ }^{104}$, P. de Jong ${ }^{105}$, L. De Mora ${ }^{71}$, M. De Oliveira Branco ${ }^{29}$, D. De Pedis ${ }^{132 a}$, A. De Salvo ${ }^{132 a}$, U. De Sanctis ${ }^{163 a, 163 c}$, A. De Santo ${ }^{148}$, J.B. De Vivie De Regie ${ }^{115}$, G. De Zorzi ${ }^{132 a, 132 b}$, S. Dean ${ }^{77}$, D.V. Dedovich ${ }^{65}$, J. Degenhardt ${ }^{120}$, M. Dehchar ${ }^{118}$, C. Del Papa ${ }^{163 a, 163 c}$, J. Del Peso ${ }^{80}$, T. Del Prete ${ }^{122 a, 122 b}$, A. Dell'Acqua ${ }^{29}$, L. Dell'Asta ${ }^{89 a, 89 b}$, M. Della Pietra ${ }^{102 a, d}$, D. della Volpe ${ }^{102 a, 102 b}$, M. Delmastro ${ }^{29}$, P.A. Delsart ${ }^{55}$, C. Deluca ${ }^{147}$, S. Demers ${ }^{174}$, M. Demichev ${ }^{65}$, B. Demirkoz ${ }^{11}$, J. Deng ${ }^{162}$, W. Deng ${ }^{24}$, S.P. Denisov ${ }^{128}$, J.E. Derkaoui ${ }^{135 c}$, F. Derue ${ }^{78}$, P. Dervan ${ }^{73}$, K. Desch ${ }^{20}$, P.O. Deviveiros ${ }^{157}$, A. Dewhurst $^{129}$, B. DeWilde ${ }^{147}$, S. Dhaliwal ${ }^{157}$, R. Dhullipudi ${ }^{24, f}$, A. Di Ciaccio ${ }^{133 a, 133 b}$, L. Di Ciaccio ${ }^{4}$, A. Di Domenico ${ }^{132 a, 132 b}$, A. Di Girolamo ${ }^{29}$, B. Di Girolamo ${ }^{29}$, S. Di Luise ${ }^{134 a, 134 b}$, A. Di Mattia ${ }^{88}$, R. Di Nardo ${ }^{133 a, 133 b}$, A. Di Simone ${ }^{133 a}$,133b, R. Di Sipio ${ }^{19 a, 19 b}$, M.A. Diaz ${ }^{31 a}$, F. Diblen ${ }^{18 c}$, E.B. Diehl ${ }^{87}$, J. Dietrich ${ }^{48}$,

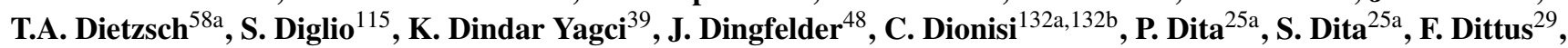
F. Djama ${ }^{83}$, R. Djilkibaev ${ }^{108}$, T. Djobava ${ }^{51}$, M.A.B. do Vale ${ }^{23 a}$, A. Do Valle Wemans ${ }^{124 a}$, T.K.O. Doan ${ }^{4}$, D. Dobos ${ }^{29}$, E. Dobson $^{29}$, M. Dobson ${ }^{162}$, C. Doglioni ${ }^{118}$, T. Doherty ${ }^{53}$, J. Dolejsi ${ }^{126}$, I. Dolenc ${ }^{74}$, Z. Dolezal ${ }^{126}$, B.A. Dolgoshein ${ }^{96}$, T. Dohmae ${ }^{154}$, M. Donega ${ }^{120}$, J. Donini ${ }^{55}$, J. Dopke ${ }^{173}$, A. Doria ${ }^{102 a}$, A. Dos Anjos ${ }^{171}$, A. Dotti ${ }^{122 a, 122 b}$, M.T. Dova ${ }^{70}$, A. Doxiadis $^{105}$, A.T. Doyle ${ }^{53}$, Z. Drasal ${ }^{126}$, M. Dris ${ }^{9}$, J. Dubbert ${ }^{99}$, E. Duchovni ${ }^{170}$, G. Duckeck ${ }^{98}$, A. Dudarev ${ }^{29}$,

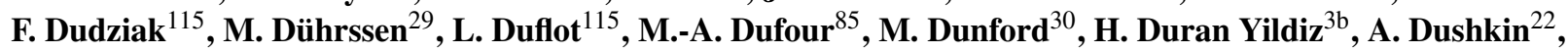
R. Duxfield ${ }^{139}$, M. Dwuznik ${ }^{37}$, M. Düren ${ }^{52}$, W.L. Ebenstein ${ }^{44}$, J. Ebke ${ }^{98}$, S. Eckweiler ${ }^{81}$, K. Edmonds ${ }^{81}$, C.A. Edwards $^{76}$, K. Egorov $^{61}$, W. Ehrenfeld ${ }^{41}$, T. Ehrich ${ }^{99}$, T. Eifert $^{29}$, G. Eigen $^{13}$, K. Einsweiler $^{14}$, E. Eisenhandler $^{75}$, T. Ekelof ${ }^{165}$, M. El Kacimi ${ }^{4}$, M. Ellert $^{165}$, S. Elles $^{4}$, F. Ellinghaus ${ }^{81}$, K. Ellis ${ }^{75}$, N. Ellis $^{29}$, J. Elmsheuser ${ }^{98}$, M. Elsing ${ }^{29}$, D. Emeliyanov ${ }^{129}$, R. Engelmann ${ }^{147}$, A. Engl ${ }^{98}$, B. Epp ${ }^{62}$, A. Eppig ${ }^{87}$, J. Erdmann ${ }^{54}$, A. Ereditato ${ }^{16}$, D. Eriksson ${ }^{145 a}$, I. Ermoline ${ }^{88}$, J. Ernst ${ }^{1}$, M. Ernst ${ }^{24}$, J. Ernwein $^{136}$, D. Errede ${ }^{164}$, S. Errede ${ }^{164}$, E. Ertel $^{81}$, M. Escalier ${ }^{115}$, C. Escobar ${ }^{166}$, X. Espinal Curull ${ }^{11}$, B. Esposito ${ }^{47}$, A.I. Etienvre $^{136}$, E. Etzion ${ }^{152}$, 
H. Evans $^{61}$, L. Fabbri ${ }^{19 a, 19 b}$, C. Fabre ${ }^{29}$, K. Facius ${ }^{35}$, R.M. Fakhrutdinov ${ }^{128}$, S. Falciano ${ }^{132 a}$, Y. Fang ${ }^{171}$, M. Fanti ${ }^{89 a, 89 b}$, A. Farbin ${ }^{7}$, A. Farilla ${ }^{134 a}$, J. Farley ${ }^{147}$, T. Farooque ${ }^{157}$, S.M. Farrington ${ }^{118}$, P. Farthouat ${ }^{29}$,

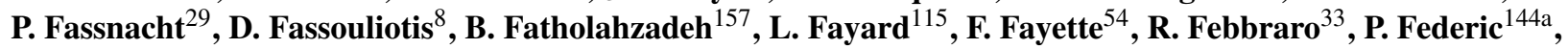
O.L. Fedin $^{121}$, W. Fedorko ${ }^{29}$, L. Feligioni ${ }^{83}$, C.U. Felzmann ${ }^{86}$, C. Feng ${ }^{32 \mathrm{~d}}$, E.J. Feng ${ }^{30}$, A.B. Fenyuk ${ }^{128}$,

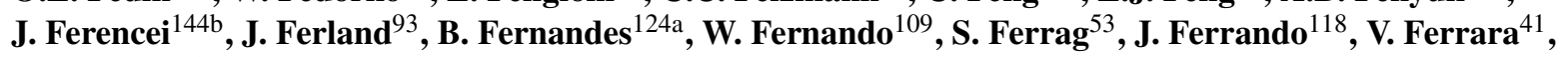

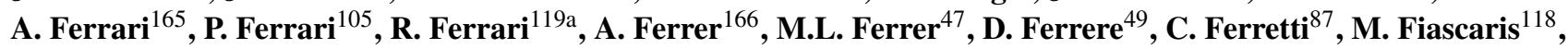
F. Fiedler ${ }^{81}$, A. Filipčič ${ }^{74}$, A. Filippas ${ }^{9}$, F. Filthaut ${ }^{104}$, M. Fincke-Keeler ${ }^{168}$, M.C.N. Fiolhais ${ }^{124}$, L. Fiorini ${ }^{11}$, A. Firan ${ }^{39}$, G. Fischer ${ }^{41}$, M.J. Fisher ${ }^{109}$, M. Flechl ${ }^{165}$, I. Fleck ${ }^{141}$, J. Fleckner ${ }^{81}$, P. Fleischmann ${ }^{172}$, S. Fleischmann ${ }^{20}$, T. Flick ${ }^{173}$, L.R. Flores Castillo ${ }^{171}$, M.J. Flowerdew ${ }^{99}$, T. Fonseca Martin $^{76}$, A. Formica ${ }^{136}$, A. Forti ${ }^{82}$, D. Fortin ${ }^{158 a}$, D. Fournier ${ }^{115}$, A.J. Fowler ${ }^{44}$, K. Fowler ${ }^{137}$, H. Fox ${ }^{71}$, P. Francavilla ${ }^{122 a, 122 b}$,

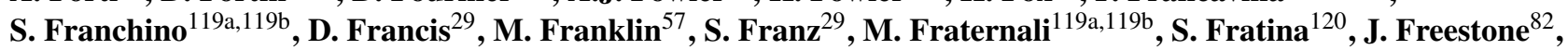
S.T. French ${ }^{27}$, R. Froeschl ${ }^{29}$, D. Froidevaux ${ }^{29}$, J.A. Frost $^{27}$, C. Fukunaga ${ }^{155}$, E. Fullana Torregrosa ${ }^{5}$, J. Fuster ${ }^{166}$,

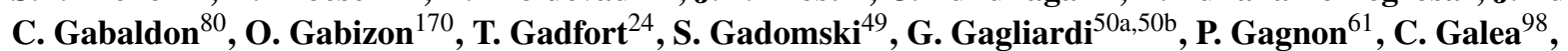
E.J. Gallas ${ }^{118}$, V. Gallo ${ }^{16}$, B.J. Gallop ${ }^{129}$, P. Gallus ${ }^{125}$, E. Galyaev ${ }^{40}$, K.K. Gan ${ }^{109}$, Y.S. Gao ${ }^{143, g}$, A. Gaponenko ${ }^{14}$, M. Garcia-Sciveres ${ }^{14}$, C. García ${ }^{166}$, J.E. García Navarro ${ }^{49}$, R.W. Gardner ${ }^{30}$, N. Garelli ${ }^{29}$, H. Garitaonandia ${ }^{105}$, V. Garonne ${ }^{29}$, C. Gatti ${ }^{47}$, G. Gaudio ${ }^{119 a}$, V. Gautard ${ }^{136}$, P. Gauzzi ${ }^{132 a, 132 b}$, I.L. Gavrilenko ${ }^{94}$, C. Gay ${ }^{167}$, G. Gaycken $^{20}$, E.N. Gazis ${ }^{9}$, P. Ge ${ }^{32 \mathrm{~d}}$, C.N.P. Gee ${ }^{129}$, Ch. Geich-Gimbel ${ }^{20}$, K. Gellerstedt ${ }^{145 a, 145 b}$, C. Gemme $^{50 a}$, M.H. Genest ${ }^{98}$, S. Gentile ${ }^{132 a, 132 b}$, F. Georgatos ${ }^{9}$, S. George ${ }^{76}$, A. Gershon ${ }^{152}$, H. Ghazlane ${ }^{135 d}$, N. Ghodbane ${ }^{33}$, B. Giacobbe ${ }^{19 a}$, S. Giagu ${ }^{132 a, 132 b}$, V. Giakoumopoulou ${ }^{8}$, V. Giangiobbe ${ }^{122 a, 122 b}$, F. Gianotti ${ }^{29}$, B. Gibbard ${ }^{24}$, A. Gibson ${ }^{157}$, S.M. Gibson ${ }^{118}$, L.M. Gilbert ${ }^{118}$, M. Gilchriese ${ }^{14}$, V. Gilewsky ${ }^{91}$, D.M. Gingrich ${ }^{2, b}$, J. Ginzburg ${ }^{152}$, N. Giokaris ${ }^{8}$, M.P. Giordani ${ }^{163 a, 163 c}$, R. Giordano ${ }^{102 a, 102 b}$, F.M. Giorgi ${ }^{15}$, P. Giovannini ${ }^{99}$, P.F. Giraud ${ }^{29}$, P. Girtler $^{62}$, D. Giugni ${ }^{89 a}$, P. Giusti ${ }^{19 a}$, B.K. Gjelsten ${ }^{117}$, L.K. Gladilin ${ }^{97}$, C. Glasman ${ }^{80}$, A. Glazov ${ }^{41}$, K.W. Glitza ${ }^{173}$, G.L. Glonti ${ }^{65}$, J. Godfrey ${ }^{142}$, J. Godlewski ${ }^{29}$, M. Goebel ${ }^{41}$, T. Göpfert ${ }^{43}$, C. Goeringer ${ }^{81}$, C. Gössling ${ }^{42}$, T. Göttfert ${ }^{99}$, V. Goggi ${ }^{19 a}$,119b,h, S. Goldfarb ${ }^{87}$, D. Goldin ${ }^{39}$, T. Golling ${ }^{174}$, A. Gomes ${ }^{124 a}$, L.S. Gomez Fajardo ${ }^{41}$, R. Gonçalo ${ }^{76}$, L. Gonella ${ }^{20}$, C. Gong ${ }^{32 b}$, S. González de la Hoz ${ }^{166}$, M.L. Gonzalez Silva ${ }^{26}$, S. Gonzalez-Sevilla ${ }^{49}$, J.J. Goodson ${ }^{147}$, L. Goossens ${ }^{29}$, H.A. Gordon ${ }^{24}$, I. Gorelov ${ }^{103}$, G. Gorfine ${ }^{173}$, B. Gorini ${ }^{29}$, E. Gorini ${ }^{\text {72a, } 72 b}$, A. Gorišek ${ }^{74}$, E. Gornicki ${ }^{38}$, B. Gosdzik ${ }^{41}$, M. Gosselink ${ }^{105}$, M.I. Gostkin ${ }^{65}$, I. Gough Eschrich ${ }^{162}$, M. Gouighri ${ }^{135 a}$, D. Goujdami ${ }^{135 a}$, M.P. Goulette ${ }^{49}$, A.G. Goussiou ${ }^{138}$, C. Goy ${ }^{4}$, I. Grabowska-Bold ${ }^{162, c}$, P. Grafström ${ }^{29}$, K.-J. Grahn ${ }^{146}$, S. Grancagnolo ${ }^{15}$, V. Grassi ${ }^{147}$, V. Gratchev ${ }^{121}$, N. Grau ${ }^{34}$, H.M. Gray ${ }^{34, i}$, J.A. Gray ${ }^{147}$, E. Graziani ${ }^{134 a}$, B. Green ${ }^{76}$, T. Greenshaw ${ }^{73}$, Z.D. Greenwood ${ }^{24, f}$, I.M. Gregor $^{41}$, P. Grenier ${ }^{143}$, E. Griesmayer ${ }^{46}$, J. Griffiths ${ }^{138}$, N. Grigalashvili6 ${ }^{65}$, A.A. Grillo ${ }^{137}$, K. Grimm ${ }^{147}$, S. Grinstein ${ }^{11}$, Y.V. Grishkevich ${ }^{97}$, M. Groh ${ }^{99}$, M. Groll ${ }^{81}$, E. Gross ${ }^{170}$, J. Grosse-Knetter ${ }^{54}$, J. Groth-Jensen ${ }^{79}$, K. Grybel ${ }^{141}$, C. Guicheney ${ }^{33}$, A. Guida ${ }^{72 a, 72 b}$,

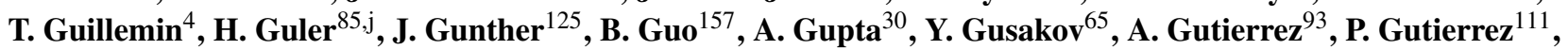
N. Guttman ${ }^{152}$, O. Gutzwiller ${ }^{171}$, C. Guyot ${ }^{136}$, C. Gwenlan ${ }^{118}$, C.B. Gwilliam ${ }^{73}$, A. Haas ${ }^{143}$, S. Haas ${ }^{29}$, C. Haber ${ }^{14}$, H.K. Hadavand ${ }^{39}$, D.R. Hadley ${ }^{17}$, P. Haefner ${ }^{99}$, R. Härtel ${ }^{99}$, Z. Hajduk ${ }^{38}$, H. Hakobyan ${ }^{175}$, J. Haller ${ }^{41, k}$,

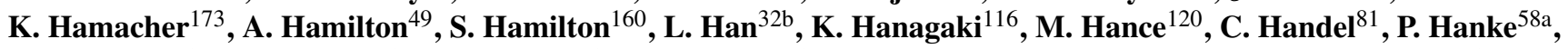

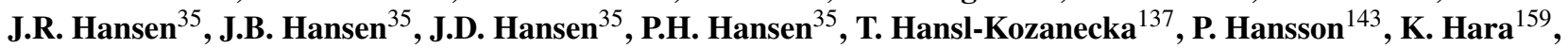

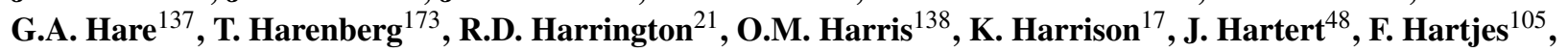

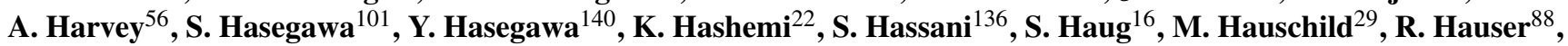

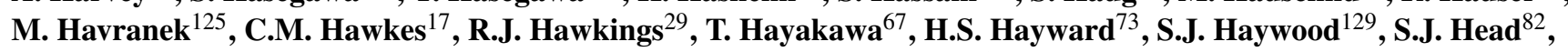
V. Hedberg ${ }^{79}$, L. Heelan ${ }^{28}$, S. Heim ${ }^{88}$, B. Heinemann ${ }^{14}$, S. Heisterkamp ${ }^{35}$, L. Helary ${ }^{4}$, M. Heller ${ }^{115}$, S. Hellman ${ }^{145 a, 145 b}$, C. Helsens ${ }^{11}$, T. Hemperek ${ }^{20}$, R.C.W. Henderson ${ }^{71}$, M. Henke ${ }^{58 a}$, A. Henrichs ${ }^{54}$, A.M. Henriques Correia ${ }^{29}$, S. Henrot-Versille ${ }^{115}$, C. Hensel $^{54}$, T. Henß ${ }^{173}$, Y. Hernández Jiménez ${ }^{166}$, A.D. Hershenhorn ${ }^{151}$, G. Herten ${ }^{48}$, R. Hertenberger ${ }^{98}$, L. Hervas $^{29}$, N.P. Hessey ${ }^{105}$, E. Higón-Rodriguez ${ }^{166}$, J.C. Hill ${ }^{27}$, K.H. Hiller ${ }^{41}$, S. Hillert ${ }^{145 a, 145 b}$, S.J. Hillier ${ }^{17}$, I. Hinchliffe ${ }^{14}$, E. Hines ${ }^{120}$, M. Hirose ${ }^{116}$, F. Hirsch ${ }^{42}$, D. Hirschbuehl $^{173}$, J. Hobbs ${ }^{147}$, N. Hod ${ }^{152}$, M.C. Hodgkinson ${ }^{139}$, P. Hodgson ${ }^{139}$, A. Hoecker ${ }^{29}$, M.R. Hoeferkamp ${ }^{103}$,

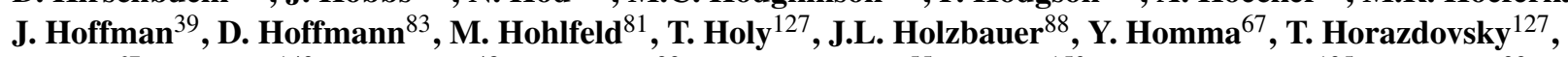
T. Hori ${ }^{67}$, C. Horn ${ }^{143}$, S. Horner ${ }^{48}$, S. Horvat ${ }^{99}$, J.-Y. Hostachy ${ }^{55}$, S. Hou ${ }^{150}$, A. Hoummada ${ }^{135 a}$, T. Howe ${ }^{39}$, J. Hrivnac $^{115}$, T. Hryn'ova ${ }^{4}$, P.J. Hsu ${ }^{174}$, S.-C. Hsu ${ }^{14}$, G.S. Huang ${ }^{111}$, Z. Hubacek ${ }^{127}$, F. Hubaut ${ }^{83}$, F. Huegging ${ }^{20}$, E.W. Hughes ${ }^{34}$, G. Hughes ${ }^{71}$, M. Hurwitz ${ }^{30}$, U. Husemann ${ }^{41}$, N. Huseynov ${ }^{10}$, J. Huston ${ }^{88}$, J. Huth ${ }^{57}$, G. Iacobucci $^{102 \mathrm{a}}$, G. Iakovidis ${ }^{9}$, I. Ibragimov ${ }^{141}$, L. Iconomidou-Fayard ${ }^{115}$, J. Idarraga ${ }^{158 b}$, P. Iengo ${ }^{4}$,

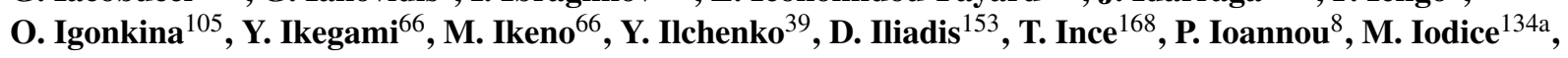


A. Irles Quiles ${ }^{166}$, A. Ishikawa ${ }^{67}$, M. Ishino ${ }^{66}$, R. Ishmukhametov ${ }^{39}$, T. Isobe ${ }^{154}$, V. Issakov $^{174, *}$, C. Issever ${ }^{118}$, S. Istin ${ }^{18 a}$, Y. Itoh ${ }^{101}$, A.V. Ivashin ${ }^{128}$, W. Iwanski ${ }^{38}$, H. Iwasaki ${ }^{66}$, J.M. Izen ${ }^{40}$, V. Izzo ${ }^{102 a}$, B. Jackson ${ }^{120}$, J.N. Jackson ${ }^{73}$, P. Jackson ${ }^{143}$, M.R. Jaekel ${ }^{29}$, V. Jain ${ }^{61}$, K. Jakobs ${ }^{48}$, S. Jakobsen ${ }^{35}$, J. Jakubek ${ }^{127}$, D.K. Jana ${ }^{111}$, E. Jansen ${ }^{104}$, A. Jantsch ${ }^{99}$, M. Janus ${ }^{48}$, R.C. Jared ${ }^{171}$, G. Jarlskog ${ }^{79}$, L. Jeanty ${ }^{57}$, I. Jen-La Plante ${ }^{30}$, P. Jenni ${ }^{29}$,

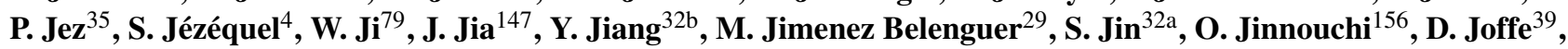
M. Johansen ${ }^{145 a, 145 b}$, K.E. Johansson ${ }^{145 a}$, P. Johansson ${ }^{139}$, S. Johnert ${ }^{41}$, K.A. Johns ${ }^{6}$, K. Jon-And ${ }^{145 a, 145 b}$, G. Jones $^{82}$, R.W.L. Jones ${ }^{71}$, T.J. Jones ${ }^{73}$, P.M. Jorge ${ }^{124 a}$, J. Joseph ${ }^{14}$, V. Juranek ${ }^{125}$, P. Jussel ${ }^{62}$, V.V. Kabachenko ${ }^{128}$, M. Kaci ${ }^{166}$, A. Kaczmarska ${ }^{38}$, M. Kado ${ }^{115}$, H. Kagan ${ }^{109}$, M. Kagan ${ }^{57}$, S. Kaiser ${ }^{99}$, E. Kajomovitz ${ }^{151}$, S. Kalinin ${ }^{173}$, L.V. Kalinovskaya ${ }^{65}$, A. Kalinowski ${ }^{130}$, S. Kama ${ }^{41}$, N. Kanaya ${ }^{154}$, M. Kaneda ${ }^{154}$, V.A. Kantserov ${ }^{96}$, J. Kanzaki ${ }^{66}$, B. Kaplan ${ }^{174}$, A. Kapliy ${ }^{30}$, J. Kaplon ${ }^{29}$, D. Kar ${ }^{43}$, M. Karagounis ${ }^{20}$, M. Karagoz Unel ${ }^{118}$, V. Kartvelishvili ${ }^{71}$, A.N. Karyukhin ${ }^{128}$, L. Kashif ${ }^{57}$, A. Kasmi ${ }^{39}$, R.D. Kass ${ }^{109}$, A. Kastanas ${ }^{13}$, M. Kastoryano ${ }^{174}$, M. Kataoka ${ }^{4}$, Y. Kataoka ${ }^{154}$, E. Katsoufis ${ }^{9}$, J. Katzy ${ }^{41}$, V. Kaushik ${ }^{6}$, K. Kawagoe ${ }^{67}$, T. Kawamoto ${ }^{154}$, G. Kawamura ${ }^{81}$, M.S. Kayl ${ }^{105}$, F. Kayumov ${ }^{94}$, V.A. Kazanin ${ }^{107}$, M.Y. Kazarinov ${ }^{65}$, J.R. Keates ${ }^{82}$, R. Keeler $^{168}$, P.T. Keener ${ }^{120}$, R. Kehoe ${ }^{39}$, M. Keil ${ }^{54}$, G.D. Kekelidze ${ }^{65}$, M. Kelly ${ }^{82}$, M. Kenyon ${ }^{53}$, O. Kepka ${ }^{125}$, N. Kerschen ${ }^{29}$, B.P. Kerševan ${ }^{74}$, S. Kersten ${ }^{173}$, K. Kessoku ${ }^{154}$, M. Khakzad ${ }^{28}$, F. Khalil-zada ${ }^{10}$, H. Khandanyan ${ }^{164}$, A. Khanov ${ }^{112}$, D. Kharchenko ${ }^{65}$, A. Khodinov ${ }^{147}$, A. Khomich ${ }^{58 a}$, G. Khoriauli ${ }^{20}$, N. Khovanskiy ${ }^{65}$, V. Khovanskiy ${ }^{95}$, E. Khramov ${ }^{65}$, J. Khubua ${ }^{51}$,

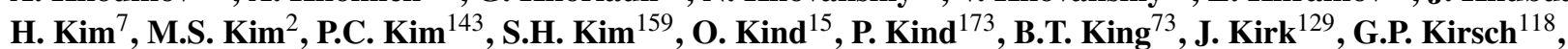
L.E. Kirsch ${ }^{22}$, A.E. Kiryunin ${ }^{99}$, D. Kisielewska ${ }^{37}$, T. Kittelmann ${ }^{123}$, H. Kiyamura ${ }^{67}$, E. Kladiva ${ }^{144 b}$, M. Klein ${ }^{73}$, U. Klein ${ }^{73}$, K. Kleinknecht ${ }^{81}$, M. Klemetti ${ }^{85}$, A. Klier ${ }^{170}$, A. Klimentov ${ }^{24}$, R. Klingenberg ${ }^{42}$, E.B. Klinkby ${ }^{44}$, T. Klioutchnikova ${ }^{29}$, P.F. Klok ${ }^{104}$, S. Klous ${ }^{105}$, E.-E. Kluge ${ }^{58 a}$, T. Kluge ${ }^{73}$, P. Kluit ${ }^{105}$, M. Klute ${ }^{54}$, S. Kluth ${ }^{99}$, N.S. Knecht ${ }^{157}$, E. Kneringer ${ }^{62}$, B.R. Ko ${ }^{44}$, T. Kobayashi ${ }^{154}$, M. Kobel ${ }^{43}$, B. Koblitz ${ }^{29}$, M. Kocian ${ }^{143}$, A. Kocnar ${ }^{113}$, P. Kodys ${ }^{126}$, K. Köneke ${ }^{41}$, A.C. König ${ }^{104}$, S. Koenig ${ }^{81}$, L. Köpke ${ }^{81}$, F. Koetsveld ${ }^{104}$, P. Koevesarki ${ }^{20}$, T. Koffas ${ }^{29}$, E. Koffeman ${ }^{105}$, F. Kohn ${ }^{54}$, Z. Kohout ${ }^{127}$, T. Kohriki' ${ }^{66}$, H. Kolanoski ${ }^{15}$, V. Kolesnikov ${ }^{65}$, I. Koletsou ${ }^{4}$, J. Koll ${ }^{88}$, D. Kollar $^{29}$, S. Kolos ${ }^{162,1}$, S.D. Kolya ${ }^{82}$, A.A. Komar ${ }^{94}$, J.R. Komaragiri ${ }^{142}$, T. Kondo ${ }^{66}$, T. Kono ${ }^{41, k}$, R. Konoplich ${ }^{108}$, S.P. Konovalov ${ }^{94}$, N. Konstantinidis ${ }^{77}$, S. Koperny ${ }^{37}$, K. Korcyl ${ }^{38}$, K. Kordas ${ }^{153}$, A. Korn ${ }^{14}$, I. Korolkov ${ }^{11}$, E.V. Korolkova ${ }^{139}$, V.A. Korotkov ${ }^{128}$, O. Kortner ${ }^{99}$, P. Kostka ${ }^{41}$, V.V. Kostyukhin ${ }^{20}$, S. Kotov ${ }^{99}$, V.M. Kotov ${ }^{65}$, K.Y. Kotov ${ }^{107}$, C. Kourkoumelis ${ }^{8}$, A. Koutsman ${ }^{105}$, R. Kowalewski ${ }^{168}$, H. Kowalski ${ }^{41}$, T.Z. Kowalski ${ }^{37}$, W. Kozanecki ${ }^{136}$, A.S. Kozhin ${ }^{128}$, V. Kral ${ }^{127}$, V.A. Kramarenko ${ }^{97}$, G. Kramberger ${ }^{74}$, M.W. Krasny ${ }^{78}$, A. Krasznahorkay ${ }^{108}$, A. Kreisel ${ }^{152}$, F. Krejci ${ }^{127}$, J. Kretzschmar ${ }^{73}$, N. Krieger ${ }^{54}$, P. Krieger ${ }^{157}$, K. Kroeninger ${ }^{54}$, H. Kroha ${ }^{99}$, J. Kroll ${ }^{120}$, J. Kroseberg ${ }^{20}$, J. Krstic ${ }^{12 a}$, U. Kruchonak ${ }^{65}$, H. Krüger ${ }^{20}$, Z.V. Krumshteyn ${ }^{65}$, T. Kubota ${ }^{154}$, S. Kuehn ${ }^{48}$, A. Kugel ${ }^{58 c}$, T. Kuhl ${ }^{173}$, D. Kuhn ${ }^{62}$, V. Kukhtin ${ }^{65}$, Y. Kulchitsky ${ }^{90}$, S. Kuleshov ${ }^{31 b}$, C. Kummer ${ }^{98}$, M. Kuna ${ }^{83}$, J. Kunkle ${ }^{120}$, A. Kupco ${ }^{125}$, H. Kurashige ${ }^{67}$, M. Kurata ${ }^{159}$, L.L. Kurchaninov ${ }^{158 a}$, Y.A. Kurochkin ${ }^{90}$, V. Kus ${ }^{125}$, R. Kwee ${ }^{15}$, L. La Rotonda ${ }^{36 a, 36 b}$, J. Labbe ${ }^{4}$, C. Lacasta $^{166}$, F. Lacava ${ }^{132 a, 132 b}$,

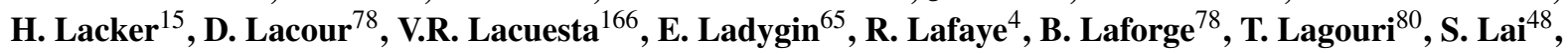
M. Lamanna ${ }^{29}$, C.L. Lampen ${ }^{6}$, W. Lampl ${ }^{6}$, E. Lancon ${ }^{136}$, U. Landgraf ${ }^{48}$, M.P.J. Landon ${ }^{75}$, J.L. Lane ${ }^{82}$,

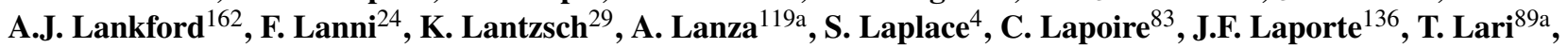

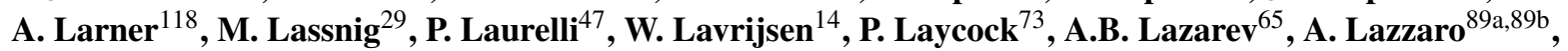

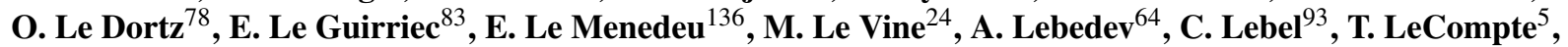

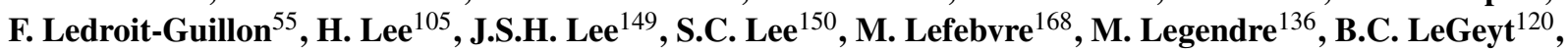
F. Legger $^{98}$, C. Leggett ${ }^{14}$, M. Lehmacher ${ }^{20}$, G. Lehmann Miotto ${ }^{29}$, X. Lei ${ }^{6}$, R. Leitner ${ }^{126}$, D. Lellouch ${ }^{170}$, J. Lellouch $^{78}$, V. Lendermann ${ }^{58 a}$, K.J.C. Leney ${ }^{73}$, T. Lenz ${ }^{173}$, G. Lenzen ${ }^{173}$, B. Lenzi ${ }^{136}$, K. Leonhardt ${ }^{43}$, C. Leroy ${ }^{93}$, J.-R. Lessard ${ }^{168}$, C.G. Lester ${ }^{27}$, A. Leung Fook Cheong ${ }^{171}$, J. Levêque ${ }^{83}$, D. Levin ${ }^{87}$, L.J. Levinson ${ }^{170}$, M. Leyton ${ }^{14}$,

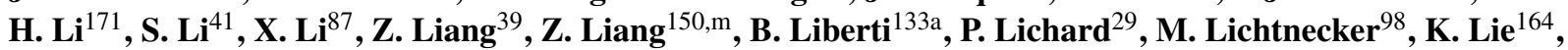
W. Liebig ${ }^{105}$, J.N. Lilley ${ }^{17}$, H. Lim ${ }^{5}$, A. Limosani ${ }^{86}$, M. Limper ${ }^{63}$, S.C. Lin ${ }^{150}$, J.T. Linnemann ${ }^{88}$, E. Lipeles ${ }^{120}$, L. Lipinsky ${ }^{125}$, A. Lipniacka ${ }^{13}$, T.M. Liss ${ }^{164}$, D. Lissauer ${ }^{24}$, A. Lister ${ }^{49}$, A.M. Litke ${ }^{137}$, C. Liu ${ }^{28}$, D. Liu ${ }^{150, n}$, H. Liu ${ }^{87}$,

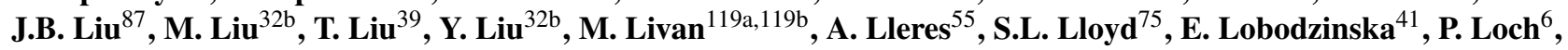
W.S. Lockman ${ }^{137}$, S. Lockwitz ${ }^{174}$, T. Loddenkoetter ${ }^{20}$, F.K. Loebinger ${ }^{82}$, A. Loginov ${ }^{174}$, C.W. Loh ${ }^{167}$, T. Lohse ${ }^{15}$,

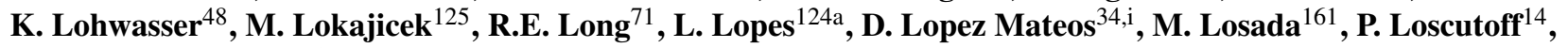

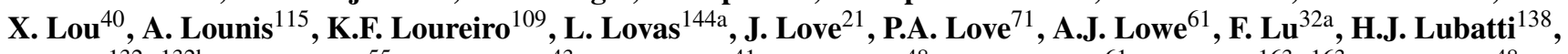
C. Luci $^{132 a, 132 b}$, A. Lucotte ${ }^{55}$, A. Ludwig ${ }^{43}$, D. Ludwig ${ }^{41}$, I. Ludwig ${ }^{48}$, F. Luehring ${ }^{61}$, L. Luisa ${ }^{163 a, 163 c}$, D. Lumb ${ }^{48}$, L. Luminari ${ }^{132 a}$, E. Lund ${ }^{117}$, B. Lund-Jensen ${ }^{146}$, B. Lundberg ${ }^{79}$, J. Lundberg ${ }^{29}$, J. Lundquist ${ }^{35}$, D. Lynn ${ }^{24}$, J. Lys ${ }^{14}$, E. Lytken ${ }^{79}$, H. Ma ${ }^{24}$, L.L. Ma ${ }^{171}$, J.A. Macana Goia ${ }^{93}$, G. Maccarrone ${ }^{47}$, A. Macchiolo ${ }^{99}$, B. Maček ${ }^{74}$, 
J. Machado Miguens ${ }^{124 a}$, R. Mackeprang ${ }^{35}$, R.J. Madaras $^{14}$, W.F. Mader ${ }^{43}$, R. Maenner ${ }^{58 c}$, T. Maeno ${ }^{24}$, P. Mättig ${ }^{173}$, S. Mättig ${ }^{41}$, P.J. Magalhaes Martins ${ }^{124 a}$, E. Magradze $^{51}$, Y. Mahalalel ${ }^{152}$, K. Mahboubi $^{48}$, A. Mahmood ${ }^{1}$, C. Maiani ${ }^{132 a, 132 b}$, C. Maidantchik ${ }^{23 a}$, A. Maio ${ }^{124 a}$, S. Majewski ${ }^{24}$, Y. Makida ${ }^{66}$, M. Makouski ${ }^{128}$, N. Makovec $^{115}$, Pa. Malecki ${ }^{38}$, P. Malecki ${ }^{38}$, V.P. Maleev ${ }^{121}$, F. Malek ${ }^{55}$, U. Mallik ${ }^{63}$, D. Malon ${ }^{5}$, S. Maltezos ${ }^{9}$, V. Malyshev ${ }^{107}$, S. Malyukov ${ }^{65}$, M. Mambelli ${ }^{30}$, R. Mameghani ${ }^{98}$, J. Mamuzic ${ }^{41}$, L. Mandelli ${ }^{89 a}$, I. Mandićc ${ }^{74}$, R. Mandrysch ${ }^{15}$, J. Maneira ${ }^{124 a}$, P.S. Mangeard ${ }^{88}$, I.D. Manjavidze ${ }^{65}$, P.M. Manning ${ }^{137}$, A. Manousakis-Katsikakis ${ }^{8}$, B. Mansoulie ${ }^{136}$, A. Mapelli ${ }^{29}$, L. Mapelli ${ }^{29}$, L. March $^{80}$, J.F. Marchand ${ }^{4}$, F. Marchese ${ }^{133 a, 133 b}$, G. Marchiori ${ }^{78}$, M. Marcisovsky ${ }^{125}$, C.P. Marino ${ }^{61}$, F. Marroquim ${ }^{23 a}$, Z. Marshall ${ }^{34, \text { i }}$, S. Marti-Garcia ${ }^{166}$, A.J. Martin ${ }^{75}$,

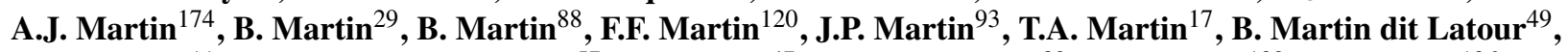
M. Martinez ${ }^{11}$, V. Martinez Outschoorn ${ }^{57}$, A. Martini ${ }^{47}$, A.C. Martyniuk $^{82}$, F. Marzano $^{132 a}$, A. Marzin ${ }^{136}$, L. Masetti $^{20}$, T. Mashimo ${ }^{154}$, R. Mashinistov ${ }^{96}$, J. Masik $^{82}$, A.L. Maslennikov ${ }^{107}$, I. Massa ${ }^{19 a, 19 b}$, N. Massol $^{4}$, A. Mastroberardino ${ }^{36 a, 36 b}$, T. Masubuchi ${ }^{154}$, P. Matricon ${ }^{115}$, H. Matsunaga ${ }^{154}$, T. Matsushita ${ }^{67}$, C. Mattravers ${ }^{18,0}$, S.J. Maxfield ${ }^{73}$, A. Mayne ${ }^{139}$, R. Mazini ${ }^{150}$, M. Mazur ${ }^{48}$, M. Mazzanti ${ }^{89 a}$, J. Mc Donald ${ }^{85}$, S.P. Mc Kee ${ }^{87}$, A. McCarn ${ }^{164}$, R.L. McCarthy ${ }^{147}$, N.A. McCubbin ${ }^{129}$, K.W. McFarlane ${ }^{56}$, H. McGlone ${ }^{53}$, G. Mchedlidze ${ }^{51}$, S.J. McMahon ${ }^{129}$, R.A. McPherson ${ }^{168, \mathrm{e}}$, A. Meade ${ }^{84}$, J. Mechnich ${ }^{105}$, M. Mechtel ${ }^{173}$, M. Medinnis ${ }^{41}$, R. Meera-Lebbai ${ }^{111}$, T.M. Meguro ${ }^{116}$, S. Mehlhase ${ }^{41}$, A. Mehta $^{73}$, K. Meier ${ }^{58 a}$, B. Meirose ${ }^{48}$, C. Melachrinos $^{30}$, B.R. Mellado Garcia ${ }^{171}$, L. Mendoza Navas ${ }^{161}$, Z. Meng ${ }^{150, p}$, S. Menke ${ }^{99}$, E. Meoni ${ }^{11}$, P. Mermod ${ }^{118}$, L. Merola ${ }^{102 a, 102 b}$, C. Meroni ${ }^{89 a}$, F.S. Merritt ${ }^{30}$, A.M. Messina ${ }^{29}$, J. Metcalfe ${ }^{103}$, A.S. Mete $^{64}$, J.-P. Meyer ${ }^{136}$,

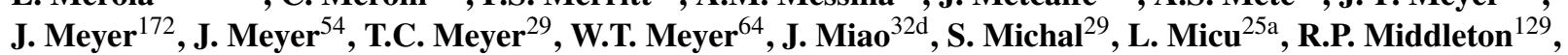
S. Migas ${ }^{73}$, L. Mijović ${ }^{74}$, G. Mikenberg ${ }^{170}$, M. Mikestikova ${ }^{125}$, M. Mikuž ${ }^{74}$, D.W. Miller ${ }^{143}$, W.J. Mills ${ }^{167}$, C.M. Mills ${ }^{57}$, A. Milov ${ }^{170}$, D.A. Milstead ${ }^{145 a, 145 b}$, D. Milstein ${ }^{170}$, A.A. Minaenko ${ }^{128}$, M. Miñano ${ }^{166}$, I.A. Minashvili ${ }^{65}$, A.I. Mincer ${ }^{108}$, B. Mindur $^{37}$, M. Mineev ${ }^{65}$, Y. Ming ${ }^{130}$, L.M. Mir ${ }^{11}$, G. Mirabelli ${ }^{132 a}$, S. Misawa ${ }^{24}$, S. Miscetti ${ }^{47}$, A. Misiejuk $^{76}$, J. Mitrevski ${ }^{137}$, V.A. Mitsou ${ }^{166}$, P.S. Miyagawa ${ }^{82}$, J.U. Mjörnmark ${ }^{79}$, D. Mladenov ${ }^{22}$, T. Moa ${ }^{145 a, 145 b}$, S. Moed $^{57}$, V. Moeller ${ }^{27}$, K. Mönig ${ }^{41}$, N. Möser ${ }^{20}$, W. Mohr ${ }^{48}$, S. Mohrdieck-Möck ${ }^{99}$, R. Moles-Valls ${ }^{166}$, J. Molina-Perez ${ }^{29}$, J. Monk ${ }^{77}$, E. Monnier ${ }^{83}$, S. Montesano ${ }^{89 a}, 89 b$, F. Monticelli ${ }^{70}$, R.W. Moore ${ }^{2}$, C. Mora Herrera ${ }^{49}$,

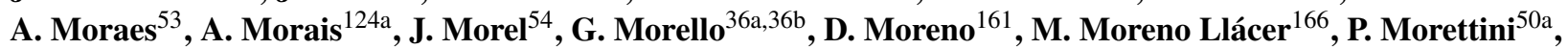
M. Morii ${ }^{57}$, A.K. Morley ${ }^{86}$, G. Mornacchi' ${ }^{29}$, S.V. Morozov ${ }^{96}$, J.D. Morris ${ }^{75}$, H.G. Moser ${ }^{99}$, M. Mosidze ${ }^{51}$, J. Moss ${ }^{109}$, R. Mount ${ }^{143}$, E. Mountricha ${ }^{136}$, S.V. Mouraviev ${ }^{94}$, E.J.W. Moyse ${ }^{84}$, M. Mudrinic ${ }^{12 b}$, F. Mueller $^{58 a}$, J. Mueller ${ }^{123}$, K. Mueller $^{20}$, T.A. Müller ${ }^{98}$, D. Muenstermann ${ }^{42}$, A. Muir ${ }^{167}$, Y. Munwes ${ }^{152}$, R. Murillo Garcia ${ }^{162}$, W.J. Murray ${ }^{129}$,

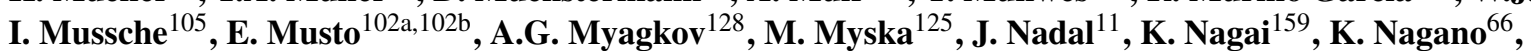
Y. Nagasaka ${ }^{60}$, A.M. Nairz ${ }^{29}$, K. Nakamura ${ }^{154}$, I. Nakano ${ }^{110}$, H. Nakatsuka ${ }^{67}$, G. Nanava ${ }^{20}$, A. Napier ${ }^{160}$,

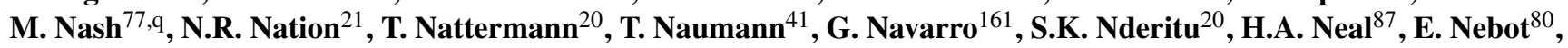
P. Nechaeva ${ }^{94}$, A. Negri ${ }^{19 a, 119 b}$, G. Negri $^{29}$, A. Nelson ${ }^{64}$, T.K. Nelson ${ }^{143}$, S. Nemecek $^{125}$, P. Nemethy $^{108}$, A.A. Nepomuceno ${ }^{23 a}$, M. Nessi ${ }^{29}$, M.S. Neubauer ${ }^{164}$, A. Neusiedl ${ }^{81}$, R.M. Neves ${ }^{108}$, P. Nevski $^{24}$, F.M. Newcomer ${ }^{120}$, R.B. Nickerson ${ }^{118}$, R. Nicolaidou ${ }^{136}$, L. Nicolas ${ }^{139}$, G. Nicoletti $^{47}$, B. Nicquevert ${ }^{29}$, F. Niedercorn ${ }^{115}$, J. Nielsen ${ }^{137}$, A. Nikiforov ${ }^{15}$, K. Nikolaev $^{65}$, I. Nikolic-Audit ${ }^{78}$, K. Nikolopoulos $^{8}$, H. Nilsen $^{48}$, P. Nilsson ${ }^{7}$, A. Nisati ${ }^{132 a}$, T. Nishiyama ${ }^{67}$, R. Nisius ${ }^{99}$, L. Nodulman ${ }^{5}$, M. Nomachi ${ }^{116}$, I. Nomidis ${ }^{153}$, M. Nordberg $^{29}$, B. Nordkvist $^{145 a, 145 b}$, D. Notz $^{41}$, J. Novakova ${ }^{126}$, M. Nozaki ${ }^{66}$, M. Nožička ${ }^{41}$, I.M. Nugent ${ }^{158 a}$, A.-E. Nuncio-Quiroz ${ }^{20}$,

G. Nunes Hanninger $^{20}$, T. Nunnemann ${ }^{98}$, E. Nurse ${ }^{77}$, D.C. O'Neil ${ }^{142}$, V. O'Shea ${ }^{53}$, F.G. Oakham ${ }^{28, b}$, H. Oberlack ${ }^{99}$, A. Ochi ${ }^{67}$, S. Oda ${ }^{154}$, S. Odaka ${ }^{66}$, J. Odier ${ }^{83}$, H. Ogren ${ }^{61}$, A. Oh $^{82}$, S.H. Oh ${ }^{44}$, C.C. Ohm ${ }^{145 a, 145 b}$, T. Ohshima ${ }^{101}$, H. Ohshita $^{140}$, T. Ohsugi ${ }^{59}$, S. Okada ${ }^{67}$, H. Okawa ${ }^{162}$, Y. Okumura ${ }^{101}$, T. Okuyama ${ }^{154}$, A.G. Olchevski ${ }^{65}$, M. Oliveira ${ }^{124 a}$, D. Oliveira Damazio ${ }^{24}$, J. Oliver ${ }^{57}$, E. Oliver Garcia ${ }^{166}$, D. Olivito ${ }^{120}$, A. Olszewski ${ }^{38}$, J. Olszowska $^{38}$, C. Omachi ${ }^{67, \mathrm{r}}$, A. Onofre ${ }^{124 a}$, P.U.E. Onyisi ${ }^{30}$, C.J. Oram ${ }^{158 a}{ }^{\text {, M.J. Oreglia }}{ }^{30}$, Y. Oren ${ }^{152}$, D. Orestano $^{134 a, 134 b}$, I. Orlov ${ }^{107}$, C. Oropeza Barrera ${ }^{53}$, R.S. Orr ${ }^{157}$, E.O. Ortega ${ }^{130}$, B. Osculati ${ }^{50,50 b}$,

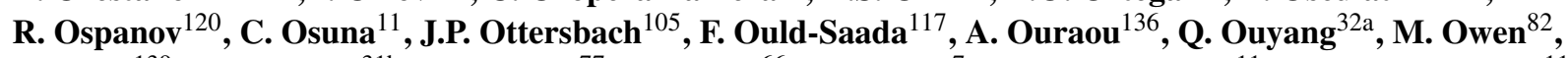
S. Owen ${ }^{139}$, A. Oyarzun ${ }^{31 b}$, V.E. Ozcan ${ }^{77}$, K. Ozone ${ }^{66}$, N. Ozturk ${ }^{7}$, A. Pacheco Pages ${ }^{11}$, C. Padilla Aranda ${ }^{11}$, E. Paganis $^{139}$, C. Pahl ${ }^{63}$, F. Paige ${ }^{24}$, K. Pajchel ${ }^{17}$, S. Palestini ${ }^{29}$, D. Pallin ${ }^{33}$, A. Palma ${ }^{124 a}$, J.D. Palmer ${ }^{17}$, Y.B. Pan ${ }^{171}$, E. Panagiotopoulou ${ }^{9}$, B. Panes ${ }^{31 a}$, N. Panikashvili ${ }^{87}$, S. Panitkin ${ }^{24}$, D. Pantea ${ }^{25 a}$, M. Panuskova ${ }^{125}$, V. Paolone ${ }^{123}$, Th.D. Papadopoulou ${ }^{9}$, S.J. Park ${ }^{54}$, W. Park ${ }^{24, \text { s } \text {, M.A. Parker }}{ }^{27}$, S.I. Parker ${ }^{14}$, F. Parodi ${ }^{50 a, 50 b}$, J.A. Parsons ${ }^{34}$, U. Parzefall ${ }^{48}$, E. Pasqualucci ${ }^{132 a}$, A. Passeri ${ }^{134 a}$, F. Pastore ${ }^{134 a, 134 b}$, Fr. Pastore ${ }^{29}$, G. Pásztor ${ }^{49, t}$,

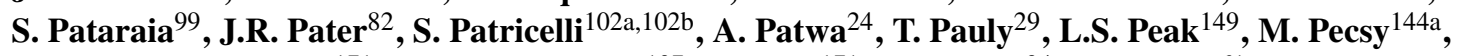
M.I. Pedraza Morales ${ }^{171}$, S.V. Peleganchuk ${ }^{107}$, H. Peng ${ }^{171}$, A. Penson ${ }^{34}$, J. Penwell ${ }^{61}$, M. Perantoni ${ }^{23 a}$, K. Perez ${ }^{34, i}$, 
E. Perez Codina ${ }^{11}$, M.T. Pérez García-Estañ ${ }^{166}$, V. Perez Reale $^{34}$, L. Perini ${ }^{89 a, 89 b}$, H. Pernegger ${ }^{29}$, R. Perrino ${ }^{72 a}$, S. Persembe ${ }^{3 a}$, P. Perus ${ }^{115}$, V.D. Peshekhonov ${ }^{65}$, B.A. Petersen ${ }^{29}$, T.C. Petersen ${ }^{35}$, E. Petit ${ }^{83}$, C. Petridou ${ }^{153}$, E. Petrolo ${ }^{132 a}$, F. Petrucci ${ }^{134 a, 134 b}$, D. Petschull ${ }^{41}$, M. Petteni ${ }^{142}$, R. Pezoa ${ }^{31 b}$, A. Phan ${ }^{86}$, A.W. Phillips ${ }^{27}$, G. Piacquadio $^{29}$, M. Piccinini ${ }^{19 a, 19 b}$, R. Piegaia ${ }^{26}$, J.E. Pilcher ${ }^{30}$, A.D. Pilkington ${ }^{82}$, J. Pina ${ }^{124 a}$, M. Pinamonti ${ }^{163 a, 163 c}$, J.L. Pinfold ${ }^{2}$, B. Pinto ${ }^{124 a}$, C. Pizio ${ }^{89 a, 89 b}$, R. Placakyte ${ }^{41}$, M. Plamondon ${ }^{168}$, M.-A. Pleier ${ }^{24}$, A. Poblaguev ${ }^{174}$, S. Poddar ${ }^{58 a}$, F. Podlyski ${ }^{33}$, P. Poffenberger ${ }^{168}$, L. Poggioli ${ }^{115}$, M. Pohl ${ }^{49}$, F. Polci ${ }^{55}$, G. Polesello ${ }^{119 a}$, A. Policicchio ${ }^{138}$, A. Polini ${ }^{19 a}$, J. Poll ${ }^{75}$, V. Polychronakos ${ }^{24}$, D. Pomeroy ${ }^{22}$, K. Pommès ${ }^{29}$, P. Ponsot ${ }^{136}$, L. Pontecorvo $^{132 a}$, B.G. Pope ${ }^{88}$, G.A. Popeneciu ${ }^{25 a}$, D.S. Popovic ${ }^{12 a}$, A. Poppleton ${ }^{29}$, J. Popule ${ }^{125}$, X. Portell Bueso ${ }^{48}$, R. Porter $^{162}$, G.E. Pospelov ${ }^{99}$, S. Pospisil ${ }^{127}$, M. Potekhin ${ }^{24}$, I.N. Potrap ${ }^{99}$, C.J. Potter ${ }^{148}$, C.T. Potter ${ }^{85}$, K.P. Potter ${ }^{82}$,

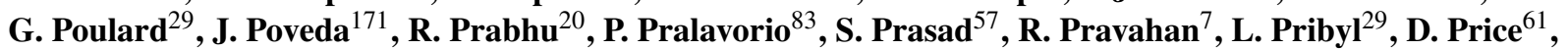
L.E. Price ${ }^{5}$, P.M. Prichard ${ }^{73}$, D. Prieur ${ }^{123}$, M. Primavera ${ }^{72 a}$, K. Prokofiev ${ }^{29}$, F. Prokoshin ${ }^{31 b}$, S. Protopopescu ${ }^{24}$, J. Proudfoot ${ }^{5}$, X. Prudent ${ }^{43}$, H. Przysiezniak ${ }^{4}$, S. Psoroulas ${ }^{20}$, E. Ptacek ${ }^{114}$, C. Puigdengoles ${ }^{11}$, J. Purdham ${ }^{87}$,

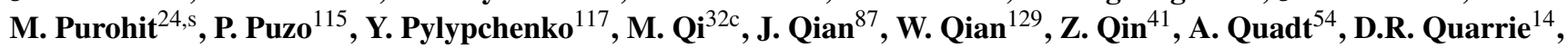

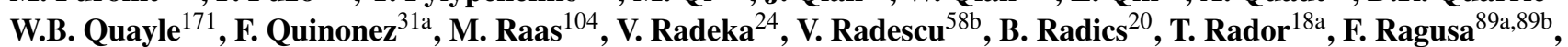
G. Rahal ${ }^{179}$, A.M. Rahimi ${ }^{109}$, S. Rajagopalan ${ }^{24}$, M. Rammensee $^{48}$, M. Rammes ${ }^{141}$, F. Rauscher ${ }^{98}$, E. Rauter ${ }^{99}$,

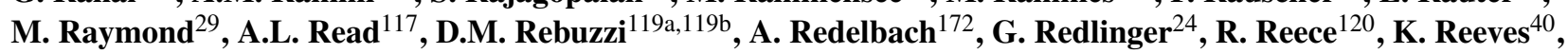
E. Reinherz-Aronis ${ }^{152}$, A. Reinsch ${ }^{114}$, I. Reisinger ${ }^{42}$, D. Reljic $^{12 a}$, C. Rembser $^{29}$, Z.L. Ren ${ }^{150}$, P. Renkel ${ }^{39}$, S. Rescia $^{24}$, M. Rescigno ${ }^{132 a}$, S. Resconi ${ }^{89 a}$, B. Resende ${ }^{136}$, P. Reznicek ${ }^{126}$, R. Rezvani ${ }^{157}$, A. Richards ${ }^{77}$, R.A. Richards ${ }^{88}$, R. Richter ${ }^{99}$, E. Richter-Was ${ }^{38, u}$, M. Ridel $^{78}$, M. Rijpstra ${ }^{105}$, M. Rijssenbeek ${ }^{147}$, A. Rimoldi ${ }^{19 a}{ }^{119 b}$, L. Rinaldi ${ }^{19 a}$, R.R. Rios $^{39}$, I. Riu ${ }^{11}$, F. Rizatdinova ${ }^{112}$, E. Rizvi ${ }^{75}$, D.A. Roa Romero ${ }^{161}$, S.H. Robertson ${ }^{85, \mathrm{e}}$, A. Robichaud-Veronneau ${ }^{49}$, D. Robinson ${ }^{27}$, J.E.M. Robinson ${ }^{77}$, M. Robinson ${ }^{114}$, A. Robson ${ }^{53}$, J.G. Rocha de Lima ${ }^{106 a}$, C. Roda ${ }^{122 a, 122 b}$, D. Roda Dos Santos ${ }^{29}$, D. Rodriguez ${ }^{161}$, Y. Rodriguez Garcia ${ }^{15}$, S. Roe $^{29}$, O. Røhne ${ }^{117}$, V. Rojo ${ }^{1}$, S. Rolli ${ }^{160}$, A. Romaniouk ${ }^{96}$, V.M. Romanov ${ }^{65}$, G. Romeo ${ }^{26}$, D. Romero Maltrana ${ }^{31 a}$,

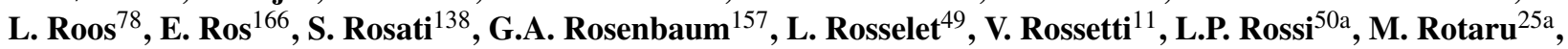
J. Rothberg ${ }^{138}$, D. Rousseau ${ }^{115}$, C.R. Royon ${ }^{136}$, A. Rozanov $^{83}$, Y. Rozen $^{151}$, X. Ruan $^{115}$, B. Ruckert $^{98}$, N. Ruckstuhl ${ }^{105}$, V.I. Rud ${ }^{97}$, G. Rudolph ${ }^{62}$, F. Rühr ${ }^{58 a}$, F. Ruggieri ${ }^{134 a}$, A. Ruiz-Martinez ${ }^{64}$, L. Rumyantsev $^{65}$,

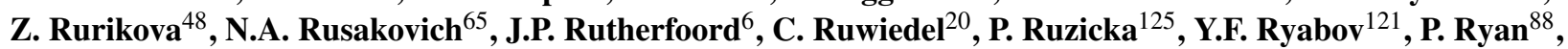
G. Rybkin ${ }^{115}$, S. Rzaeva ${ }^{10}$, A.F. Saavedra ${ }^{149}$, H.F.-W. Sadrozinski ${ }^{137}$, R. Sadykov ${ }^{65}$, H. Sakamoto ${ }^{154}$, G. Salamanna $^{105}$, A. Salamon ${ }^{133 a}$, M.S. Saleem ${ }^{111}$, D. Salihagic ${ }^{99}$, A. Salnikov ${ }^{143}$, J. Salt ${ }^{166}$, B.M. Salvachua Ferrando $^{5}$, D. Salvatore ${ }^{36 a, 36 b}$, F. Salvatore ${ }^{148}$, A. Salvucci ${ }^{47}$, A. Salzburger ${ }^{29}$, D. Sampsonidis ${ }^{153}$, B.H. Samset ${ }^{117}$, H. Sandaker ${ }^{13}$, H.G. Sander ${ }^{81}$, M.P. Sanders ${ }^{98}$, M. Sandhoff ${ }^{173}$, P. Sandhu ${ }^{157}$, R. Sandstroem ${ }^{105}$, S. Sandvoss ${ }^{173}$,

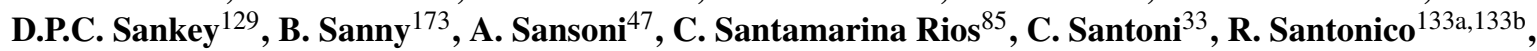
J.G. Saraiva ${ }^{124 a}$, T. Sarangi ${ }^{171}$, E. Sarkisyan-Grinbaum ${ }^{7}$, F. Sarri ${ }^{122 a, 122 b}$, O. Sasaki $^{66}$, N. Sasao ${ }^{68}$, I. Satsounkevitch ${ }^{90}$, G. Sauvage ${ }^{4}$, P. Savard ${ }^{157, b}$, A.Y. Savine ${ }^{6}$, V. Savinov ${ }^{123}$, L. Sawyer ${ }^{24, f}$, D.H. Saxon ${ }^{53}$, L.P. Says ${ }^{33}$, C. Sbarra ${ }^{19 a, 19 b}$, A. Sbrizzi ${ }^{19 a, 19 b}$, D.A. Scannicchio ${ }^{29}$, J. Schaarschmidt ${ }^{43}$, P. Schacht ${ }^{99}$, U. Schäfer ${ }^{81}$, S. Schaetzel ${ }^{58 b}$, A.C. Schaffer ${ }^{115}$, D. Schaile ${ }^{98}$, R.D. Schamberger ${ }^{147}$, A.G. Schamov ${ }^{107}$, V.A. Schegelsky ${ }^{121}$, D. Scheirich ${ }^{87}$, M. Schernau ${ }^{162}$, M.I. Scherzer ${ }^{14}$, C. Schiavi $^{50 a, 50 b}$, J. Schieck ${ }^{99}$, M. Schioppa ${ }^{36 a, 36 b}$, S. Schlenker ${ }^{29}$, K. Schmieden ${ }^{20}$, C. Schmitt ${ }^{81}$, M. Schmitz ${ }^{20}$, M. Schott ${ }^{29}$, D. Schouten ${ }^{142}$, J. Schovancova ${ }^{125}$, M. Schram ${ }^{85}$, A. Schreiner ${ }^{63}$, C. Schroeder ${ }^{81}$, N. Schroer ${ }^{58 c}$, M. Schroers ${ }^{173}$, J. Schultes ${ }^{173}$, H.-C. Schultz-Coulon ${ }^{58 a}$, J.W. Schumacher ${ }^{43}$, M. Schumacher ${ }^{48}$, B.A. Schumm ${ }^{137}$, Ph. Schune ${ }^{136}$, C. Schwanenberger ${ }^{82}$, A. Schwartzman ${ }^{143}$, Ph. Schwemling ${ }^{78}$, R. Schwienhorst ${ }^{88}$, R. Schwierz ${ }^{43}$, J. Schwindling ${ }^{136}$, W.G. Scott $^{129}$, J. Searcy ${ }^{114}$, E. Sedykh ${ }^{121}$, E. Segura ${ }^{11}$, S.C. Seidel ${ }^{103}$, A. Seiden ${ }^{137}$, F. Seifert ${ }^{43}$, J.M. Seixas ${ }^{23 a}$, G. Sekhniaidze ${ }^{102 a}$, D.M. Seliverstov ${ }^{121}$, B. Sellden ${ }^{15 a}$, N. Semprini-Cesari ${ }^{19 a, 19 b}$, C. Serfon ${ }^{98}$, L. Serin $^{115}$, R. Seuster ${ }^{99}$, H. Severini ${ }^{111}$, M.E. Sevior ${ }^{86}$, A. Sfyrla ${ }^{164}$, E. Shabalina ${ }^{54}$, M. Shamim ${ }^{114}$, L.Y. Shan ${ }^{32 a}$, J.T. Shank ${ }^{21}$, Q.T. Shao ${ }^{86}$, M. Shapiro ${ }^{14}$, P.B. Shatalov ${ }^{95}$, K. Shaw ${ }^{139}$, D. Sherman ${ }^{29}$, P. Sherwood ${ }^{77}$, A. Shibata ${ }^{108}$, M. Shimojima ${ }^{100}$, T. Shin ${ }^{56}$, A. Shmeleva ${ }^{94}$, M.J. Shochet ${ }^{30}$, M.A. Shupe ${ }^{6}$, P. Sicho ${ }^{125}$, A. Sidoti ${ }^{15}$, F. Siegert ${ }^{77}$, J. Siegrist ${ }^{14}$, Dj. Sijacki ${ }^{12 a}$, O. Silbert ${ }^{170}$, J. Silva ${ }^{124 a}$, Y. Silver ${ }^{152}$, D. Silverstein ${ }^{143}$, S.B. Silverstein ${ }^{145 a}$, V. Simak ${ }^{127}$, Lj. Simic ${ }^{12 a}$, S. Simion ${ }^{115}$, B. Simmons ${ }^{77}$, M. Simonyan ${ }^{35}$, P. Sinervo ${ }^{157}$, N.B. Sinev ${ }^{114}$, V. Sipica ${ }^{141}$, G. Siragusa ${ }^{81}$, A.N. Sisakyan ${ }^{65}$, S.Yu. Sivoklokov ${ }^{97}$, J. Sjoelin ${ }^{145 a, 145 b}$, T.B. Sjursen ${ }^{13}$, K. Skovpen ${ }^{107}$, P. Skubic ${ }^{111}$, M. Slater ${ }^{17}$, T. Slavicek ${ }^{127}$, K. Sliwa ${ }^{160}$, J. Sloper ${ }^{29}$, T. Sluka ${ }^{125}$, V. Smakhtin ${ }^{170}$, S.Yu. Smirnov ${ }^{96}$, Y. Smirnov ${ }^{24}$, L.N. Smirnova ${ }^{97}$, O. Smirnova ${ }^{79}$, B.C. Smith ${ }^{57}$, D. Smith ${ }^{143}$, K.M. Smith ${ }^{53}$, M. Smizanska ${ }^{71}$, K. Smolek ${ }^{127}$, A.A. Snesarev ${ }^{94}$, S.W. Snow ${ }^{82}$, J. Snow ${ }^{111}$, J. Snuverink ${ }^{105}$, S. Snyder ${ }^{24}$, M. Soares ${ }^{124 a}$, R. Sobie ${ }^{168, \text { e }}$, J. Sodomka ${ }^{127}$, A. Soffer ${ }^{152}$, C.A. Solans ${ }^{166}$, M. Solar ${ }^{127}$, 
J. Solc $^{127}$, E. Solfaroli Camillocci ${ }^{132 a, 132 b}$, A.A. Solodkov ${ }^{128}$, O.V. Solovyanov ${ }^{128}$, R. Soluk ${ }^{2}$, J. Sondericker ${ }^{24}$, V. Sopko ${ }^{127}$, B. Sopko ${ }^{127}$, M. Sosebee ${ }^{7}$, A. Soukharev ${ }^{107}$, S. Spagnolo ${ }^{72 a, 72 b}$, F. Spanò ${ }^{34}$, E. Spencer ${ }^{137}$, R. Spighi ${ }^{19 a}$, G. Spigo $^{29}$, F. Spila ${ }^{132 a, 132 b}$, R. Spiwoks ${ }^{29}$, M. Spousta ${ }^{126}$, T. Spreitzer ${ }^{142}$, B. Spurlock ${ }^{7}$, R.D. St. Denis ${ }^{53}$, T. Stahl ${ }^{141}$, J. Stahlman ${ }^{120}$, R. Stamen ${ }^{58 a}$, S.N. Stancu ${ }^{162}$, E. Stanecka ${ }^{29}$, R.W. Stanek ${ }^{5}$, C. Stanescu $^{134 a}$, S. Stapnes ${ }^{117}$,

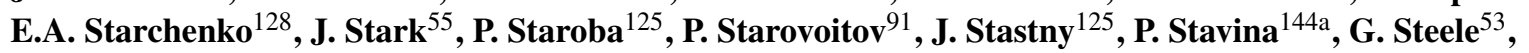
P. Steinbach ${ }^{43}$, P. Steinberg ${ }^{24}$, I. Stekl ${ }^{127}$, B. Stelzer ${ }^{142}$, H.J. Stelzer ${ }^{41}$, O. Stelzer-Chilton $^{158 a}$, H. Stenzel ${ }^{52}$, K. Stevenson ${ }^{75}$, G.A. Stewart ${ }^{53}$, M.C. Stockton ${ }^{29}$, K. Stoerig $^{48}$, G. Stoicea ${ }^{25 a}$, S. Stonjek ${ }^{99}$, P. Strachota ${ }^{126}$, A.R. Stradling ${ }^{7}$, A. Straessner ${ }^{43}$, J. Strandberg ${ }^{87}$, S. Strandberg ${ }^{14}$, A. Strandlie ${ }^{117}$, M. Strauss ${ }^{111}$, P. Strizenec ${ }^{144 b}$, R. Ströhmer ${ }^{172}$, D.M. Strom ${ }^{114}$, R. Stroynowski ${ }^{39}$, J. Strube ${ }^{129}$, B. Stugu ${ }^{13}$, D.A. Soh ${ }^{150, v}$, D. Su ${ }^{143}$, Y. Sugaya ${ }^{116}$, T. Sugimoto ${ }^{101}$, C. Suhr ${ }^{106 a}$, M. Suk ${ }^{126}$, V.V. Sulin ${ }^{94}$, S. Sultansoy ${ }^{3 \mathrm{~d}}$, T. Sumida ${ }^{29}$, X.H. Sun ${ }^{32 \mathrm{~d}}$, J.E. Sundermann ${ }^{48}$, K. Suruliz ${ }^{163 a, 163 b}$, S. Sushkov ${ }^{11}$, G. Susinno ${ }^{36,36 b}$, M.R. Sutton ${ }^{139}$, T. Suzuki ${ }^{154}$, Y. Suzuki ${ }^{66}$, I. Sykora ${ }^{144 a}$, T. Sykora ${ }^{126}$, T. Szymocha ${ }^{38}$, J. Sánchez ${ }^{166}$, D. Ta ${ }^{20}$, K. Tackmann ${ }^{29}$, A. Taffard ${ }^{162}$, R. Tafirout ${ }^{158 a}$, A. Taga ${ }^{117}$, Y. Takahashi $^{101}$, H. Takai ${ }^{24}$, R. Takashima ${ }^{69}$, H. Takeda ${ }^{67}$, T. Takeshita ${ }^{140}$, M. Talby $^{83}$, A. Talyshev ${ }^{107}$, M.C. Tamsett $^{76}$, J. Tanaka ${ }^{154}$, R. Tanaka ${ }^{115}$, S. Tanaka ${ }^{131}$, S. Tanaka ${ }^{66}$, S. Tapprogge ${ }^{81}$, D. Tardif $^{157}$, S. Tarem $^{151}$, F. Tarrade ${ }^{24}$, G.F. Tartarelli ${ }^{89 a}$, P. Tas ${ }^{126}$, M. Tasevsky ${ }^{125}$, E. Tassi ${ }^{36 a, 36 b}$, M. Tatarkhanov ${ }^{14}$, C. Taylor ${ }^{77}$, F.E. Taylor ${ }^{22}$, G.N. Taylor ${ }^{86}$, R.P. Taylor ${ }^{168}$, W. Taylor ${ }^{158 b}$, P. Teixeira-Dias ${ }^{76}$, H. Ten Kate ${ }^{29}$, P.K. Teng ${ }^{150}$,

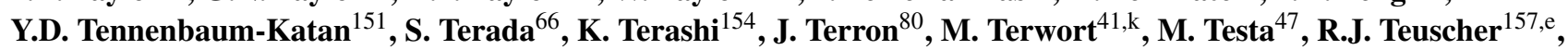
M. Thioye ${ }^{174}$, S. Thoma ${ }^{48}$, J.P. Thomas ${ }^{17}$, E.N. Thompson ${ }^{84}$, P.D. Thompson ${ }^{17}$, P.D. Thompson ${ }^{157}$, R.J. Thompson ${ }^{82}$, A.S. Thompson ${ }^{53}$, E. Thomson ${ }^{120}$, R.P. Thun ${ }^{87}$, T. Tic ${ }^{125}$, V.O. Tikhomirov ${ }^{94}$, Y.A. Tikhonov ${ }^{107}$, P. Tipton ${ }^{174}$, F.J. Tique Aires Viegas ${ }^{29}$, S. Tisserant ${ }^{83}$, B. Toczek $^{37}$, T. Todorov ${ }^{4}$, S. Todorova-Nova ${ }^{160}$, B. Toggerson ${ }^{162}$, J. Tojo ${ }^{66}$, S. Tokár ${ }^{144 a}$, K. Tokushuku ${ }^{66}$, K. Tollefson ${ }^{88}$, L. Tomasek ${ }^{125}$, M. Tomasek ${ }^{125}$, M. Tomoto ${ }^{101}$, L. Tompkins ${ }^{14}$, K. Toms ${ }^{103}$, A. Tonoyan ${ }^{13}$, C. Topfel ${ }^{16}$, N.D. Topilin ${ }^{65}$, E. Torrence ${ }^{114}$, E. Torró Pastor ${ }^{166}$, J. Toth ${ }^{83, \mathrm{t}}$, F. Touchard ${ }^{83}$, D.R. Tovey ${ }^{139}$, T. Trefzger ${ }^{172}$, L. Tremblet ${ }^{29}$, A. Tricoli ${ }^{29}$, I.M. Trigger ${ }^{158 a}$, S. Trincaz-Duvoid ${ }^{78}$, T.N. Trinh ${ }^{78}$,

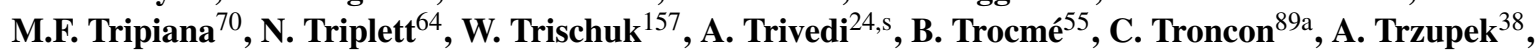
C. Tsarouchas ${ }^{9}$, J.C.-L. Tseng ${ }^{118}$, M. Tsiakiris ${ }^{105}$, P.V. Tsiareshka ${ }^{90}$, D. Tsionou ${ }^{139}$, G. Tsipolitis ${ }^{9}$, V. Tsiskaridze ${ }^{51}$, E.G. Tskhadadze ${ }^{51}$, I.I. Tsukerman ${ }^{95}$, V. Tsulaia ${ }^{123}$, J.-W. Tsung ${ }^{20}$, S. Tsuno ${ }^{66}$, D. Tsybychev ${ }^{147}$, J.M. Tuggle ${ }^{30}$, D. Turecek ${ }^{127}$, I. Turk Cakir ${ }^{3 e}$, E. Turlay ${ }^{105}$, P.M. Tuts ${ }^{34}$, M.S. Twomey ${ }^{138}$, M. Tylmad ${ }^{145 a, 145 b}$, M. Tyndel ${ }^{129}$, K. Uchida ${ }^{116}$, I. Ueda ${ }^{154}$, M. Ugland ${ }^{13}$, M. Uhlenbrock ${ }^{20}$, M. Uhrmacher ${ }^{54}$, F. Ukegawa ${ }^{159}$, G. Unal ${ }^{29}$, A. Undrus $^{24}$,

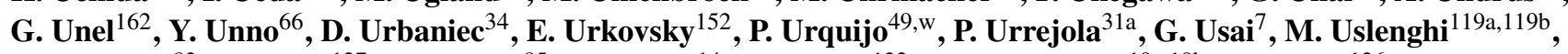
L. Vacavant ${ }^{83}$, V. Vacek ${ }^{127}$, B. Vachon ${ }^{85}$, S. Vahsen ${ }^{14}$, P. Valente $^{132 a}$, S. Valentinetti ${ }^{19 a, 19 b}$, S. Valkar ${ }^{126}$, E. Valladolid Gallego ${ }^{166}$, S. Vallecorsa ${ }^{151}$, J.A. Valls Ferrer ${ }^{166}$, R. Van Berg ${ }^{120}$, H. van der Graaf ${ }^{105}$, E. van der Kraaij ${ }^{105}$, E. van der Poel ${ }^{105}$, D. van der Ster $^{29}$, N. van Eldik ${ }^{84}$, P. van Gemmeren ${ }^{5}$, Z. van Kesteren ${ }^{105}$, I. van Vulpen ${ }^{105}$, W. Vandelli ${ }^{29}$, A. Vaniachine ${ }^{5}$, P. Vankov $^{73}$, F. Vannucci ${ }^{78}$, R. Vari $^{132 a}$, E.W. Varnes ${ }^{6}$, D. Varouchas ${ }^{14}$, A. Vartapetian ${ }^{7}$, K.E. Varvell ${ }^{149}$, L. Vasilyeva ${ }^{94}$, V.I. Vassilakopoulos ${ }^{56}$, F. Vazeille ${ }^{33}$, C. Vellidis ${ }^{8}$, F. Veloso ${ }^{124 a}$, S. Veneziano ${ }^{132 a}$, A. Ventura ${ }^{72 a, 72 b}$, D. Ventura ${ }^{138}$, M. Venturi ${ }^{48}$, N. Venturi ${ }^{16}$, V. Vercesi $^{119 a}$, M. Verducci ${ }^{172}$, W. Verkerke ${ }^{105}$, J.C. Vermeulen ${ }^{105}$, M.C. Vetterli ${ }^{142, b}$, I. Vichou ${ }^{164}$, T. Vickey ${ }^{118}$, G.H.A. Viehhauser ${ }^{118}$, M. Villa ${ }^{19 a, 19 b}$, E.G. Villani ${ }^{129}$, M. Villaplana Perez ${ }^{166}$, E. Vilucchi ${ }^{47}$, M.G. Vincter ${ }^{28}$,

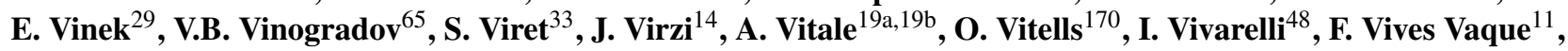

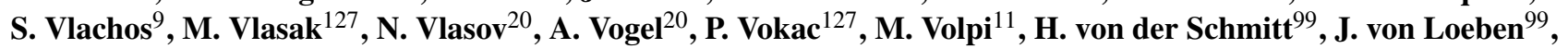
H. von Radziewski ${ }^{48}$, E. von Toerne ${ }^{20}$, V. Vorobel ${ }^{126}$, V. Vorwerk $^{11}$, M. Vos ${ }^{166}$, R. Voss ${ }^{29}$, T.T. Voss ${ }^{173}$, J.H. Vossebeld ${ }^{73}$, N. Vranjes ${ }^{12 a}$, M. Vranjes Milosavljevic ${ }^{12 a}$, V. Vrba ${ }^{125}$, M. Vreeswijk ${ }^{105}$, T. Vu Anh ${ }^{81}$,

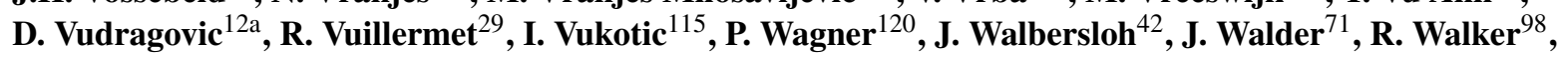
W. Walkowiak ${ }^{141}$, R. Wall ${ }^{174}$, C. Wang ${ }^{44}$, H. Wang ${ }^{171}$, J. Wang ${ }^{55}$, S.M. Wang ${ }^{150}$, A. Warburton ${ }^{85}$, C.P. Ward ${ }^{27}$, M. Warsinsky ${ }^{48}$, R. Wastie ${ }^{118}$, P.M. Watkins ${ }^{17}$, A.T. Watson ${ }^{17}$, M.F. Watson ${ }^{17}$, G. Watts $^{138}$, S. Watts ${ }^{82}$, A.T. Waugh ${ }^{149}$, B.M. Waugh ${ }^{77}$, M.D. Weber ${ }^{16}$, M. Weber ${ }^{129}$, M.S. Weber ${ }^{16}$, P. Weber $^{58 a}$, A.R. Weidberg ${ }^{118}$, J. Weingarten ${ }^{54}$, C. Weiser ${ }^{48}$, H. Wellenstein ${ }^{22}$, P.S. Wells ${ }^{29}$, M. Wen ${ }^{47}$, T. Wenaus ${ }^{24}$, S. Wendler ${ }^{123}$, T. Wengler ${ }^{82}$,

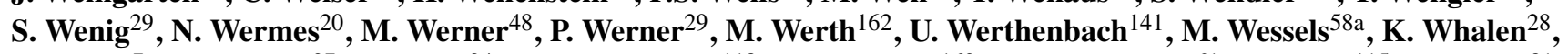
A. White ${ }^{7}$, M.J. White ${ }^{27}$, S. White ${ }^{24}$, S.R. Whitehead ${ }^{118}$, D. Whiteson ${ }^{162}$, D. Whittington ${ }^{61}$, F. Wicek ${ }^{115}$, D. Wicke ${ }^{81}$, F.J. Wickens ${ }^{129}$, W. Wiedenmann ${ }^{171}$, M. Wielers ${ }^{129}$, P. Wienemann ${ }^{20}$, C. Wiglesworth ${ }^{73}$, L.A.M. Wiik ${ }^{48}$, A. Wildauer ${ }^{166}$, M.A. Wildt ${ }^{41, k}$, H.G. Wilkens ${ }^{29}$, E. Williams ${ }^{34}$, H.H. Williams ${ }^{120}$, S. Willocq ${ }^{84}$, J.A. Wilson ${ }^{17}$, M.G. Wilson ${ }^{143}$, A. Wilson ${ }^{87}$, I. Wingerter-Seez ${ }^{4}$, F. Winklmeier $^{29}$, M. Wittgen ${ }^{143}$, M.W. Wolter ${ }^{38}$, H. Wolters ${ }^{124 a}$,

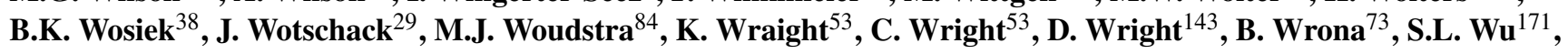




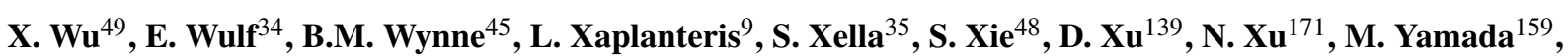
A. Yamamoto ${ }^{66}$, K. Yamamoto $^{64}$, S. Yamamoto ${ }^{154}$, T. Yamamura ${ }^{154}$, J. Yamaoka ${ }^{44}$, T. Yamazaki ${ }^{154}$, Y. Yamazaki ${ }^{67}$,

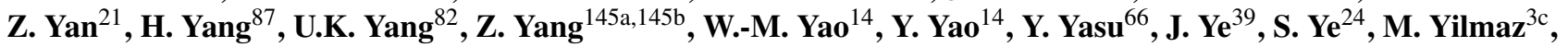
R. Yoosoofmiya ${ }^{123}$, K. Yorita ${ }^{169}$, R. Yoshida ${ }^{5}$, C. Young ${ }^{143}$, S.P. Youssef ${ }^{21}$, D. Yu ${ }^{24}$, J. Yu ${ }^{7}$, L. Yuan ${ }^{78}$, A. Yurkewicz ${ }^{147}$, R. Zaidan ${ }^{63}$, A.M. Zaitsev ${ }^{128}$, Z. Zajacova ${ }^{29}$, V. Zambrano ${ }^{47}$, L. Zanello ${ }^{132 a, 132 b}$, A. Zaytsev ${ }^{107}$, C. Zeitnitz ${ }^{173}$, M. Zeller ${ }^{174}$, A. Zemla ${ }^{38}$, C. Zendler ${ }^{20}$, O. Zenin ${ }^{128}$, T. Zenis ${ }^{144 a}$, Z. Zenonos ${ }^{122 a, 122 b}$, S. Zenz ${ }^{14}$, D. Zerwas ${ }^{115}$, G. Zevi della Porta ${ }^{57}$, Z. Zhan ${ }^{32 \mathrm{~d}}$, H. Zhang ${ }^{83}$, J. Zhang ${ }^{5}$, Q. Zhang ${ }^{5}$, X. Zhang ${ }^{32 \mathrm{~d}}$, L. Zhao ${ }^{108}$, T. Zhao ${ }^{138}$, Z. Zhao ${ }^{32 b}$, A. Zhemchugov ${ }^{65}$, J. Zhong ${ }^{150, x}$, B. Zhou ${ }^{87}$, N. Zhou ${ }^{34}$, Y. Zhou ${ }^{150}$, C.G. Zhu ${ }^{32 \mathrm{~d}}$, H. Zhu ${ }^{41}$, Y. Zhu ${ }^{171}$, X. Zhuang ${ }^{98}$, V. Zhuravlov ${ }^{99}$, R. Zimmermann ${ }^{20}$, S. Zimmermann ${ }^{20}$, S. Zimmermann ${ }^{48}$, M. Ziolkowski ${ }^{141}$, L. Živkovićc ${ }^{34}$, G. Zobernig ${ }^{171}$, A. Zoccoli ${ }^{19 a, 19 b}$, M. zur Nedden ${ }^{15}$, V. Zutshi ${ }^{106 a}$

${ }^{\star}$ CERN, 1211 Genève 23, Switzerland

${ }^{1}$ University at Albany, 1400 Washington Ave, Albany, NY 12222, United States of America

${ }^{2}$ University of Alberta, Department of Physics, Centre for Particle Physics, Edmonton, AB T6G 2G7, Canada

${ }^{3}$ Ankara University ${ }^{(a)}$, Faculty of Sciences, Department of Physics, TR 061000 Tandogan, Ankara; Dumlupinar University ${ }^{(b)}$, Faculty of Arts and Sciences, Department of Physics, Kutahya; Gazi University ${ }^{(\mathrm{c})}$, Faculty of Arts and Sciences, Department of Physics, 06500,

Teknikokullar, Ankara; TOBB University of Economics and Technology ${ }^{(\mathrm{d})}$, Faculty of Arts and Sciences, Division of Physics, 06560 , Sogutozu, Ankara; Turkish Atomic Energy Authority ${ }^{(\mathrm{e})}$, 06530, Lodumlu, Ankara, Turkey

${ }^{4}$ LAPP, Université de Savoie, CNRS/IN2P3, Annecy-le-Vieux, France

${ }^{5}$ Argonne National Laboratory, High Energy Physics Division, 9700 S. Cass Avenue, Argonne IL 60439, United States of America

${ }^{6}$ University of Arizona, Department of Physics, Tucson, AZ 85721, United States of America

${ }^{7}$ The University of Texas at Arlington, Department of Physics, Box 19059, Arlington, TX 76019, United States of America

${ }^{8}$ University of Athens, Nuclear \& Particle Physics, Department of Physics, Panepistimiopouli, Zografou, GR 15771 Athens, Greece

${ }^{9}$ National Technical University of Athens, Physics Department, 9-Iroon Polytechniou, GR 15780 Zografou, Greece

${ }^{10}$ Institute of Physics, Azerbaijan Academy of Sciences, H. Javid Avenue 33, AZ 143 Baku, Azerbaijan

${ }^{11}$ Institut de Física d'Altes Energies, IFAE, Edifici Cn, Universitat Autònoma de Barcelona, ES-08193 Bellaterra (Barcelona), Spain

${ }^{12}$ University of Belgrade ${ }^{(a)}$, Institute of Physics, P.O. Box 57, 11001 Belgrade; Vinca Institute of Nuclear Sciences ${ }^{(b)}$, Mihajla Petrovica Alasa 12-14, 11001 Belgrade, Serbia

${ }^{13}$ University of Bergen, Department for Physics and Technology, Allegaten 55, NO-5007 Bergen, Norway

${ }^{14}$ Lawrence Berkeley National Laboratory and University of California, Physics Division, MS50B-6227, 1 Cyclotron Road, Berkeley, CA 94720, United States of America

${ }^{15}$ Humboldt University, Institute of Physics, Berlin, Newtonstr. 15, D-12489 Berlin, Germany

${ }^{16}$ University of Bern, Albert Einstein Center for Fundamental Physics, Laboratory for High Energy Physics, Sidlerstrasse 5, CH-3012 Bern, Switzerland

${ }^{17}$ University of Birmingham, School of Physics and Astronomy, Edgbaston, Birmingham B15 2TT, United Kingdom

${ }^{18}$ Bogazici University $^{(\mathrm{a})}$, Faculty of Sciences, Department of Physics, TR-80815 Bebek-Istanbul; Dogus University ${ }^{(\mathrm{b})}$, Faculty of Arts and Sciences, Department of Physics, 34722, Kadikoy, Istanbul; ${ }^{\left({ }^{c}\right)}$ Gaziantep University, Faculty of Engineering, Department of Physics Engineering, 27310, Sehitkamil, Gaziantep, Turkey; Istanbul Technical University ${ }^{(\mathrm{d})}$, Faculty of Arts and Sciences, Department of Physics, 34469, Maslak, Istanbul, Turkey

${ }^{19}$ INFN Sezione di Bologna ${ }^{(\mathrm{a})}$; Università di Bologna, Dipartimento di Fisica ${ }^{(\mathrm{b})}$, viale C. Berti Pichat, 6/2, IT - 40127 Bologna, Italy

${ }^{20}$ University of Bonn, Physikalisches Institut, Nussallee 12, D-53115 Bonn, Germany

${ }^{21}$ Boston University, Department of Physics, 590 Commonwealth Avenue, Boston, MA 02215, United States of America

${ }^{22}$ Brandeis University, Department of Physics, MS057, 415 South Street, Waltham, MA 02454, United States of America

${ }^{23}$ Universidade Federal do Rio De Janeiro, COPPE/EE/IF ${ }^{(a)}$, Caixa Postal 68528, Ilha do Fundao, BR-21945-970 Rio de Janeiro;

(b) Universidade de Sao Paulo, Instituto de Fisica, R.do Matao Trav. R.187, Sao Paulo-SP, 05508-900, Brazil

${ }^{24}$ Brookhaven National Laboratory, Physics Department, Bldg. 510A, Upton, NY 11973, United States of America

${ }^{25}$ National Institute of Physics and Nuclear Engineering (a), Bucharest-Magurele, Str. Atomistilor 407, P.O. Box MG-6, R-077125, Romania; University Politehnica Bucharest $^{(\mathrm{b})}$, Rectorat-AN 001, 313 Splaiul Independentei, sector 6, 060042 Bucuresti; West University ${ }^{(\mathrm{c})}$ in Timisoara, Bd. Vasile Parvan 4, Timisoara, Romania

${ }^{26}$ Universidad de Buenos Aires, FCEyN, Dto. Fisica, Pab I-C. Universitaria, 1428 Buenos Aires, Argentina

${ }^{27}$ University of Cambridge, Cavendish Laboratory, J.J. Thomson Avenue, Cambridge CB3 OHE, United Kingdom

${ }^{28}$ Carleton University, Department of Physics, 1125 Colonel By Drive, Ottawa ON K1S 5B6, Canada

${ }^{29} \mathrm{CERN}, \mathrm{CH}-1211$ Geneva 23, Switzerland

${ }^{30}$ University of Chicago, Enrico Fermi Institute, 5640 S. Ellis Avenue, Chicago, IL 60637, United States of America

${ }^{31}$ Pontificia Universidad Católica de Chile, Facultad de Fisica, Departamento de Fisica ${ }^{(a)}$, Avda. Vicuna Mackenna 4860, San Joaquin, Santiago; Universidad Técnica Federico Santa María, Departamento de Física ${ }^{(b)}$, Avda. Espãna 1680, Casilla 110-V, Valparaíso, Chile

${ }^{32}$ Institute of High Energy Physics, Chinese Academy of Sciences ${ }^{(a)}$, P.O. Box 918, 19 Yuquan Road, Shijing Shan District, CN-Beijing 100049; University of Science \& Technology of China (USTC), Department of Modern Physics ${ }^{(b)}$, Hefei, CN-Anhui 230026; Nanjing University, Department of Physics $^{(\mathrm{c})}, 22$ Hankou Road, Nanjing, 210093; Shandong University, High Energy Physics Group ${ }^{(\mathrm{d})}$, Jinan, CN-Shandong 250100, China

${ }^{33}$ Laboratoire de Physique Corpusculaire, Clermont Université, Université Blaise Pascal, CNRS/IN2P3, FR-63177 Aubiere Cedex, France

${ }^{34}$ Columbia University, Nevis Laboratory, 136 So. Broadway, Irvington, NY 10533, United States of America

${ }^{35}$ University of Copenhagen, Niels Bohr Institute, Blegdamsvej 17, DK-2100 Kobenhavn 0, Denmark

${ }^{36}$ INFN Gruppo Collegato di Cosenza ${ }^{(a)}$; Università della Calabria, Dipartimento di Fisica ${ }^{(b)}$, IT-87036 Arcavacata di Rende, Italy 
${ }^{37}$ Faculty of Physics and Applied Computer Science of the AGH-University of Science and Technology (FPACS, AGH-UST), al. Mickiewicza 30, PL-30059 Cracow, Poland

${ }^{38}$ The Henryk Niewodniczanski Institute of Nuclear Physics, Polish Academy of Sciences, ul. Radzikowskiego 152, PL-31342 Krakow, Poland

${ }^{39}$ Southern Methodist University, Physics Department, 106 Fondren Science Building, Dallas, TX 75275-0175, United States of America

${ }^{40}$ University of Texas at Dallas, 800 West Campbell Road, Richardson, TX 75080-3021, United States of America

${ }^{41}$ DESY, Notkestr. 85, D-22603 Hamburg and Platanenallee 6, D-15738 Zeuthen, Germany

${ }^{42}$ TU Dortmund, Experimentelle Physik IV, DE-44221 Dortmund, Germany

${ }^{43}$ Technical University Dresden, Institut für Kern- und Teilchenphysik, Zellescher Weg 19, D-01069 Dresden, Germany

${ }^{44}$ Duke University, Department of Physics, Durham, NC 27708, United States of America

${ }^{45}$ University of Edinburgh, School of Physics \& Astronomy, James Clerk Maxwell Building, The Kings Buildings, Mayfield Road, Edinburgh EH9 3JZ, United Kingdom

${ }^{46}$ Fachhochschule Wiener Neustadt; Johannes Gutenbergstrasse 3 AT-2700 Wiener Neustadt, Austria

${ }^{47}$ INFN Laboratori Nazionali di Frascati, via Enrico Fermi 40, IT-00044 Frascati, Italy

${ }^{48}$ Albert-Ludwigs-Universität, Fakultät für Mathematik und Physik, Hermann-Herder Str. 3, D-79104 Freiburg i.Br., Germany

${ }^{49}$ Université de Genève, Section de Physique, 24 rue Ernest Ansermet, CH-1211 Geneve 4, Switzerland

${ }^{50}$ INFN Sezione di Genova $^{(a)}$; Università di Genova, Dipartimento di Fisica ${ }^{(b)}$, via Dodecaneso 33, IT-16146 Genova, Italy

${ }^{51}$ Institute of Physics of the Georgian Academy of Sciences, 6 Tamarashvili St., GE-380077 Tbilisi; Tbilisi State University, HEP Institute, University St. 9, GE-380086 Tbilisi, Georgia

${ }^{52}$ Justus-Liebig-Universität Giessen, II Physikalisches Institut, Heinrich-Buff Ring 16, D-35392 Giessen, Germany

${ }^{53}$ University of Glasgow, Department of Physics and Astronomy, Glasgow G12 8QQ, United Kingdom

${ }^{54}$ Georg-August-Universität, II. Physikalisches Institut, Friedrich-Hund Platz 1, D-37077 Göttingen, Germany

${ }^{55}$ Laboratoire de Physique Subatomique et de Cosmologie, CNRS/IN2P3, Université Joseph Fourier, INPG, 53 avenue des Martyrs, FR-38026 Grenoble Cedex, France

${ }^{56}$ Hampton University, Department of Physics, Hampton, VA 23668, United States of America

${ }^{57}$ Harvard University, Laboratory for Particle Physics and Cosmology, 18 Hammond Street, Cambridge, MA 02138, United States of America

${ }^{58}$ Ruprecht-Karls-Universität Heidelberg: Kirchhoff-Institut für Physik ${ }^{(a)}$, Im Neuenheimer Feld 227, D-69120 Heidelberg; Physikalisches Institut $^{(\mathrm{b})}$, Philosophenweg 12, D-69120 Heidelberg; ZITI Ruprecht-Karls-University Heidelberg ${ }^{(\mathrm{c})}$, Lehrstuhl für Informatik V, B6, 23-29, DE-68131 Mannheim, Germany

${ }^{59}$ Hiroshima University, Faculty of Science, 1-3-1 Kagamiyama, Higashihiroshima-shi, JP-Hiroshima 739-8526, Japan

${ }^{60}$ Hiroshima Institute of Technology, Faculty of Applied Information Science, 2-1-1 Miyake Saeki-ku, Hiroshima-shi, JP-Hiroshima 731-5193, Japan

${ }^{61}$ Indiana University, Department of Physics, Swain Hall West 117, Bloomington, IN 47405-7105, United States of America

${ }^{62}$ Institut für Astro- und Teilchenphysik, Technikerstrasse 25, A-6020 Innsbruck, Austria

${ }^{63}$ University of Iowa, 203 Van Allen Hall, Iowa City, IA 52242-1479, United States of America

${ }^{64}$ Iowa State University, Department of Physics and Astronomy, Ames High Energy Physics Group, Ames, IA 50011-3160, United States of America

${ }^{65}$ Joint Institute for Nuclear Research, JINR Dubna, RU-141 980 Moscow Region, Russia

${ }^{66}$ KEK, High Energy Accelerator Research Organization, 1-1 Oho, Tsukuba-shi, Ibaraki-ken 305-0801, Japan

${ }^{67}$ Kobe University, Graduate School of Science, 1-1 Rokkodai-cho, Nada-ku, JP Kobe 657-8501, Japan

${ }^{68}$ Kyoto University, Faculty of Science, Oiwake-cho, Kitashirakawa, Sakyou-ku, Kyoto-shi, JP-Kyoto 606-8502, Japan

${ }^{69}$ Kyoto University of Education, 1 Fukakusa, Fujimori, Fushimi-ku, Kyoto-shi, JP-Kyoto 612-8522, Japan

${ }^{70}$ Universidad Nacional de La Plata, FCE, Departamento de Física, IFLP (CONICET-UNLP), C.C. 67, 1900 La Plata, Argentina

${ }^{71}$ Lancaster University, Physics Department, Lancaster LA1 4YB, United Kingdom

${ }^{72}$ INFN Sezione di Lecce ${ }^{(\mathrm{a})}$; Università del Salento, Dipartimento di Fisica ${ }^{(\mathrm{b})}$ Via Arnesano IT-73100 Lecce, Italy

${ }^{73}$ University of Liverpool, Oliver Lodge Laboratory, P.O. Box 147, Oxford Street, Liverpool L69 3BX, United Kingdom

${ }^{74}$ Jožef Stefan Institute and University of Ljubljana, Department of Physics, SI-1000 Ljubljana, Slovenia

${ }^{75}$ Queen Mary University of London, Department of Physics, Mile End Road, London E1 4NS, United Kingdom

${ }^{76}$ Royal Holloway, University of London, Department of Physics, Egham Hill, Egham, Surrey TW20 0EX, United Kingdom

${ }^{77}$ University College London, Department of Physics and Astronomy, Gower Street, London WC1E 6BT, United Kingdom

${ }^{78}$ Laboratoire de Physique Nucléaire et de Hautes Energies, Université Pierre et Marie Curie (Paris 6), Université Denis Diderot (Paris-7), CNRS/IN2P3, Tour 33, 4 place Jussieu, FR-75252 Paris Cedex 05, France

${ }^{79}$ Lunds Universitet, Naturvetenskapliga Fakulteten, Fysiska Institutionen, Box 118, SE-221 00 Lund, Sweden

${ }^{80}$ Universidad Autonoma de Madrid, Facultad de Ciencias, Departamento de Fisica Teorica, ES-28049 Madrid, Spain

${ }^{81}$ Universität Mainz, Institut für Physik, Staudinger Weg 7, DE-55099 Mainz, Germany

${ }^{82}$ University of Manchester, School of Physics and Astronomy, Manchester M13 9PL, United Kingdom

${ }^{83}$ CPPM, Aix-Marseille Université, CNRS/IN2P3, Marseille, France

${ }^{84}$ University of Massachusetts, Department of Physics, 710 North Pleasant Street, Amherst, MA 01003, United States of America

${ }^{85}$ McGill University, High Energy Physics Group, 3600 University Street, Montreal, Quebec H3A 2T8, Canada

${ }^{86}$ University of Melbourne, School of Physics, AU-Parkville, Victoria 3010, Australia

${ }^{87}$ The University of Michigan, Department of Physics, 2477 Randall Laboratory, 500 East University, Ann Arbor, MI 48109-1120, United States of America

${ }^{88}$ Michigan State University, Department of Physics and Astronomy, High Energy Physics Group, East Lansing, MI 48824-2320, United States of America

${ }^{89}$ INFN Sezione di Milano $^{(a)}$; Università di Milano, Dipartimento di Fisica ${ }^{(b)}$, via Celoria 16, IT-20133 Milano, Italy

${ }^{90}$ B.I. Stepanov Institute of Physics, National Academy of Sciences of Belarus, Independence Avenue 68, Minsk 220072, Republic of Belarus 
${ }^{91}$ National Scientific \& Educational Centre for Particle \& High Energy Physics, NC PHEP BSU, M. Bogdanovich St. 153, Minsk 220040, Republic of Belarus

${ }^{92}$ Massachusetts Institute of Technology, Department of Physics, Room 24-516, Cambridge, MA 02139, United States of America

${ }^{93}$ University of Montreal, Group of Particle Physics, C.P. 6128, Succursale Centre-Ville, Montreal, Quebec, H3C 3J7, Canada

${ }^{94}$ P.N. Lebedev Institute of Physics, Academy of Sciences, Leninsky pr. 53, RU-117 924 Moscow, Russia

${ }^{95}$ Institute for Theoretical and Experimental Physics (ITEP), B. Cheremushkinskaya ul. 25, RU 117218 Moscow, Russia

${ }^{96}$ Moscow Engineering \& Physics Institute (MEPhI), Kashirskoe Shosse 31, RU-115409 Moscow, Russia

${ }^{97}$ Lomonosov Moscow State University Skobeltsyn Institute of Nuclear Physics (MSU SINP), 1(2), Leninskie gory, GSP-1, Moscow 119991 Russian Federation, Russia

${ }^{98}$ Ludwig-Maximilians-Universität München, Fakultät für Physik, Am Coulombwall 1, DE-85748 Garching, Germany

${ }^{99}$ Max-Planck-Institut für Physik (Werner-Heisenberg-Institut), Föhringer Ring 6, 80805 München, Germany

${ }^{100}$ Nagasaki Institute of Applied Science, 536 Aba-machi, JP Nagasaki 851-0193, Japan

${ }^{101}$ Nagoya University, Graduate School of Science, Furo-Cho, Chikusa-ku, Nagoya, 464-8602, Japan

${ }^{102}$ INFN Sezione di Napoli ${ }^{(a)}$; Università di Napoli, Dipartimento di Scienze Fisiche ${ }^{(\mathrm{b})}$, Complesso Universitario di Monte Sant'Angelo, via Cinthia, IT-80126 Napoli, Italy

${ }^{103}$ University of New Mexico, Department of Physics and Astronomy, MSC07 4220, Albuquerque, NM 87131 USA, United States of America

${ }^{104}$ Radboud University Nijmegen/NIKHEF, Department of Experimental High Energy Physics, Heyendaalseweg 135, NL-6525 AJ, Nijmegen, Netherlands

${ }^{105}$ Nikhef National Institute for Subatomic Physics, and University of Amsterdam, Science Park 105, 1098 XG Amsterdam, Netherlands

106(a) DeKalb, Illinois 60115, United States of America

${ }^{107}$ Budker Institute of Nuclear Physics (BINP), RU-Novosibirsk 630 090, Russia

${ }^{108}$ New York University, Department of Physics, 4 Washington Place, New York NY 10003, USA, United States of America

${ }^{109}$ Ohio State University, 191 West Woodruff Ave, Columbus, OH 43210-1117, United States of America

${ }^{110}$ Okayama University, Faculty of Science, Tsushimanaka 3-1-1, Okayama 700-8530, Japan

${ }^{111}$ University of Oklahoma, Homer L. Dodge Department of Physics and Astronomy, 440 West Brooks, Room 100, Norman, OK 73019-0225, United States of America

${ }^{112}$ Oklahoma State University, Department of Physics, 145 Physical Sciences Building, Stillwater, OK 74078-3072, United States of America

${ }^{113}$ Palacký University, 17. listopadu 50a, 77207 Olomouc, Czech Republic

${ }^{114}$ University of Oregon, Center for High Energy Physics, Eugene, OR 97403-1274, United States of America

${ }^{115}$ LAL, Univ. Paris-Sud, IN2P3/CNRS, Orsay, France

${ }^{116}$ Osaka University, Graduate School of Science, Machikaneyama-machi 1-1, Toyonaka, Osaka 560-0043, Japan

${ }^{117}$ University of Oslo, Department of Physics, P.O. Box 1048, Blindern, NO-0316 Oslo 3, Norway

${ }^{118}$ Oxford University, Department of Physics, Denys Wilkinson Building, Keble Road, Oxford OX1 3RH, United Kingdom

${ }^{119}$ INFN Sezione di Pavia ${ }^{(a)}$; Università di Pavia, Dipartimento di Fisica Nucleare e Teorica ${ }^{(b)}$, Via Bassi 6, IT-27100 Pavia, Italy

${ }^{120}$ University of Pennsylvania, Department of Physics, High Energy Physics Group, 209 S. 33rd Street, Philadelphia, PA 19104, United States of America

${ }^{121}$ Petersburg Nuclear Physics Institute, RU-188 300 Gatchina, Russia

${ }^{122}$ INFN Sezione di Pisa ${ }^{(a)}$; Università di Pisa, Dipartimento di Fisica E. Fermi ${ }^{(b)}$, Largo B. Pontecorvo 3, IT-56127 Pisa, Italy

${ }^{123}$ University of Pittsburgh, Department of Physics and Astronomy, 3941 O'Hara Street, Pittsburgh, PA 15260, United States of America

${ }^{124}$ Laboratorio de Instrumentacao e Fisica Experimental de Particulas-LIP ${ }^{(a)}$, Avenida Elias Garcia 14-1, PT-1000-149 Lisboa, Portugal; Universidad de Granada, Departamento de Fisica Teorica y del Cosmos and CAFPE ${ }^{(\mathrm{b})}$, E-18071 Granada, Spain

${ }^{125}$ Institute of Physics, Academy of Sciences of the Czech Republic, Na Slovance 2, CZ-18221 Praha 8, Czech Republic

${ }^{126}$ Charles University in Prague, Faculty of Mathematics and Physics, Institute of Particle and Nuclear Physics, V Holesovickach 2, CZ-18000 Praha 8, Czech Republic

${ }^{127}$ Czech Technical University in Prague, Zikova 4, CZ-166 35 Praha 6, Czech Republic

${ }^{128}$ State Research Center Institute for High Energy Physics, Moscow Region, 142281, Protvino, Pobeda street, 1, Russia

${ }^{129}$ Rutherford Appleton Laboratory, Science and Technology Facilities Council, Harwell Science and Innovation Campus, Didcot OX11 0QX, United Kingdom

${ }^{130}$ University of Regina, Physics Department, Canada

${ }^{131}$ Ritsumeikan University, Noji Higashi 1 chome 1-1, JP-Kusatsu, Shiga 525-8577, Japan

${ }^{132}$ INFN Sezione di Roma I ${ }^{(a)}$; Università La Sapienza, Dipartimento di Fisica ${ }^{(\text {b) }}$, Piazzale A. Moro 2, IT-00185 Roma, Italy

${ }^{133}$ INFN Sezione di Roma Tor Vergata ${ }^{(a)}$; Università di Roma Tor Vergata, Dipartimento di Fisica ${ }^{(b)}$, via della Ricerca Scientifica, IT-00133 Roma, Italy

${ }^{134}$ INFN Sezione di Roma Tre ${ }^{(a)}$; Università Roma Tre, Dipartimento di Fisica ${ }^{(b)}$, via della Vasca Navale 84, IT-00146 Roma, Italy

${ }^{135}$ Réseau Universitaire de Physique des Hautes Energies (RUPHE): Université Hassan II, Faculté des Sciences Ain Chock ${ }^{(\mathrm{a})}$, B.P. 5366, MA-Casablanca; Centre National de 1'Energie des Sciences Techniques Nucleaires (CNESTEN) $)^{\text {(b) }}$, B.P. 1382 R.P. 10001 Rabat 10001 ; Université Mohamed Premier ${ }^{(\mathrm{c})}$, LPTPM, Faculté des Sciences, B.P.717. Bd. Mohamed VI, 60000, Oujda; Université Mohammed V, Faculté des Sciences $^{(\mathrm{d})}$, 4 Avenue Ibn Battouta, BP 1014 RP, 10000 Rabat, Morocco

${ }^{136} \mathrm{CEA}$, DSM/IRFU, Centre d'Etudes de Saclay, FR-91191 Gif-sur-Yvette, France

${ }^{137}$ University of California Santa Cruz, Santa Cruz Institute for Particle Physics (SCIPP), Santa Cruz, CA 95064, United States of America

${ }^{138}$ University of Washington, Seattle, Department of Physics, Box 351560, Seattle, WA 98195-1560, United States of America

${ }^{139}$ University of Sheffield, Department of Physics \& Astronomy, Hounsfield Road, Sheffield S3 7RH, United Kingdom

${ }^{140}$ Shinshu University, Department of Physics, Faculty of Science, 3-1-1 Asahi, Matsumoto-shi, JP-Nagano 390-8621, Japan

${ }^{141}$ Universität Siegen, Fachbereich Physik, D 57068 Siegen, Germany

${ }^{142}$ Simon Fraser University, Department of Physics, 8888 University Drive, CA-Burnaby, BC V5A 1S6, Canada 
${ }^{143}$ SLAC National Accelerator Laboratory, Stanford, California 94309, United States of America

${ }^{144}$ Comenius University, Faculty of Mathematics, Physics \& Informatics ${ }^{(a)}$, Mlynska dolina F2, SK-84248 Bratislava; Institute of Experimental Physics of the Slovak Academy of Sciences, Dept. of Subnuclear Physics ${ }^{(b)}$, Watsonova 47, SK-04353 Kosice, Slovak Republic

${ }^{145}$ Stockholm University: Department of Physics ${ }^{(a)}$; The Oskar Klein Centre ${ }^{(b)}$, AlbaNova, SE-106 91 Stockholm, Sweden

${ }^{146}$ Royal Institute of Technology (KTH), Physics Department, SE-106 91 Stockholm, Sweden

${ }^{147}$ Stony Brook University, Department of Physics and Astronomy, Nicolls Road, Stony Brook, NY 11794-3800, United States of America

${ }^{148}$ University of Sussex, Department of Physics and Astronomy Pevensey 2 Building, Falmer, Brighton BN1 9QH, United Kingdom

${ }^{149}$ University of Sydney, School of Physics, AU-Sydney NSW 2006, Australia

${ }^{150}$ Insitute of Physics, Academia Sinica, TW-Taipei 11529, Taiwan

${ }^{151}$ Technion, Israel Inst. of Technology, Department of Physics, Technion City, IL-Haifa 32000, Israel

${ }^{152}$ Tel Aviv University, Raymond and Beverly Sackler School of Physics and Astronomy, Ramat Aviv, IL-Tel Aviv 69978, Israel

${ }^{153}$ Aristotle University of Thessaloniki, Faculty of Science, Department of Physics, Division of Nuclear \& Particle Physics, University Campus, GR-54124, Thessaloniki, Greece

${ }^{154}$ The University of Tokyo, International Center for Elementary Particle Physics and Department of Physics, 7-3-1 Hongo, Bunkyo-ku, JP-Tokyo 113-0033, Japan

${ }^{155}$ Tokyo Metropolitan University, Graduate School of Science and Technology, 1-1 Minami-Osawa, Hachioji, Tokyo 192-0397, Japan

${ }^{156}$ Tokyo Institute of Technology, 2-12-1-H-34 O-Okayama, Meguro, Tokyo 152-8551, Japan

${ }^{157}$ University of Toronto, Department of Physics, 60 Saint George Street, Toronto M5S 1A7, Ontario, Canada

${ }^{158}$ TRIUMF $^{(a)}$, 4004 Wesbrook Mall, Vancouver, B.C. V6T 2A3; (b) York University, Department of Physics and Astronomy, 4700 Keele St., Toronto, Ontario, M3J 1P3, Canada

${ }^{159}$ University of Tsukuba, Institute of Pure and Applied Sciences, 1-1-1 Tennoudai, Tsukuba-shi, JP-Ibaraki 305-8571, Japan

${ }^{160}$ Tufts University, Science \& Technology Center, 4 Colby Street, Medford, MA 02155, United States of America

${ }^{161}$ Universidad Antonio Narino, Centro de Investigaciones, Cra 3 Este No. 47A-15, Bogota, Colombia

${ }^{162}$ University of California, Irvine, Department of Physics \& Astronomy, CA 92697-4575, United States of America

${ }^{163}$ INFN Gruppo Collegato di Udine ${ }^{(\mathrm{a})} ;$ ICTP $^{(\mathrm{b})}$, Strada Costiera 11, IT-34014, Trieste; Università di Udine, Dipartimento di Fisica ${ }^{(\mathrm{c})}$, via delle Scienze 208, IT-33100 Udine, Italy

${ }^{164}$ University of Illinois, Department of Physics, 1110 West Green Street, Urbana, Illinois 61801, United States of America

${ }^{165}$ University of Uppsala, Department of Physics and Astronomy, P.O. Box 516, SE-51 20 Uppsala, Sweden

${ }^{166}$ Instituto de Física Corpuscular (IFIC) Centro Mixto UVEG-CSIC, Apdo. 22085 ES-46071 Valencia, Dept. Física At. Mol. y Nuclear; Univ. of Valencia, and Instituto de Microelectrónica de Barcelona (IMB-CNM-CSIC) 08193 Bellaterra Barcelona, Spain

${ }^{167}$ University of British Columbia, Department of Physics, 6224 Agricultural Road, CA-Vancouver, B.C. V6T 1Z1, Canada

${ }^{168}$ University of Victoria, Department of Physics and Astronomy, P.O. Box 3055, Victoria B.C., V8W 3P6, Canada

${ }^{169}$ Waseda University, WISE, 3-4-1 Okubo, Shinjuku-ku, Tokyo, 169-8555, Japan

${ }^{170}$ The Weizmann Institute of Science, Department of Particle Physics, P.O. Box 26, IL-76100 Rehovot, Israel

${ }^{171}$ University of Wisconsin, Department of Physics, 1150 University Avenue, WI 53706 Madison, Wisconsin, United States of America

${ }^{172}$ Julius-Maximilians-University of Würzburg, Physikalisches Institute, Am Hubland, 97074 Würzburg, Germany

${ }^{173}$ Bergische Universität, Fachbereich C, Physik, Postfach 100127, Gauss-Strasse 20, D-42097 Wuppertal, Germany

${ }^{174}$ Yale University, Department of Physics, PO Box 208121, New Haven CT, 06520-8121, United States of America

${ }^{175}$ Yerevan Physics Institute, Alikhanian Brothers Street 2, AM-375036 Yerevan, Armenia

${ }^{176}$ ATLAS-Canada Tier-1 Data Centre, TRIUMF, 4004 Wesbrook Mall, Vancouver, BC, V6T 2A3, Canada

${ }^{177}$ GridKA Tier-1 FZK, Forschungszentrum Karlsruhe GmbH, Steinbuch Centre for Computing (SCC), Hermann-von-Helmholtz-Platz 1, 76344 Eggenstein-Leopoldshafen, Germany

${ }^{178}$ Port d'Informacio Cientifica (PIC), Universitat Autonoma de Barcelona (UAB), Edifici D, E-08193 Bellaterra, Spain

${ }^{179}$ Centre de Calcul CNRS/IN2P3, Domaine Scientifique de la Doua, 27 bd du 11 Novembre 1918, 69622 Villeurbanne Cedex, France

${ }^{180}$ INFN-CNAF, Viale Berti Pichat 6/2, 40127 Bologna, Italy

${ }^{181}$ Nordic Data Grid Facility, NORDUnet A/S, Kastruplundgade 22, 1, DK-2770 Kastrup, Denmark

${ }^{182}$ SARA Reken- en Netwerkdiensten, Science Park 121, 1098 XG Amsterdam, Netherlands

${ }^{183}$ Academia Sinica Grid Computing, Institute of Physics, Academia Sinica, No.128, Sec. 2, Academia Rd., Nankang, Taipei, Taiwan 11529, Taiwan

${ }^{184}$ UK-T1-RAL Tier-1, Rutherford Appleton Laboratory, Science and Technology Facilities Council, Harwell Science and Innovation Campus, Didcot OX11 0QX, United Kingdom

${ }^{185}$ RHIC and ATLAS Computing Facility, Physics Department, Building 510, Brookhaven National Laboratory, Upton, New York 11973 , United States of America

${ }^{\mathrm{a}}$ Also at CPPM, Marseille, France.

${ }^{\mathrm{b}}$ Also at TRIUMF, 4004 Wesbrook Mall, Vancouver, B.C. V6T 2A3, Canada

${ }^{\mathrm{c}}$ Also at Faculty of Physics and Applied Computer Science of the AGH-University of Science and Technology (FPACS, AGH-UST), al. Mickiewicza 30, PL-30059 Cracow, Poland

${ }^{\mathrm{d}}$ Also at Università di Napoli Parthenope, via A. Acton 38, IT-80133 Napoli, Italy

eAlso at Institute of Particle Physics (IPP), Canada

${ }^{\mathrm{f}}$ Louisiana Tech University, 305 Wisteria Street, P.O. Box 3178, Ruston, LA 71272, United States of America

${ }^{g}$ At Department of Physics, California State University, Fresno, 2345 E. San Ramon Avenue, Fresno, CA 93740-8031, United States of America

${ }^{\mathrm{h}}$ Currently at Istituto Universitario di Studi Superiori IUSS, V.le Lungo Ticino Sforza 56, 27100 Pavia, Italy

${ }^{\mathrm{i}}$ Also at California Institute of Technology, Physics Department, Pasadena, CA 91125, United States of America

${ }^{j}$ Also at University of Montreal, Canada

${ }^{k}$ Also at Institut für Experimentalphysik, Universität Hamburg, Luruper Chaussee 149, 22761 Hamburg, Germany 


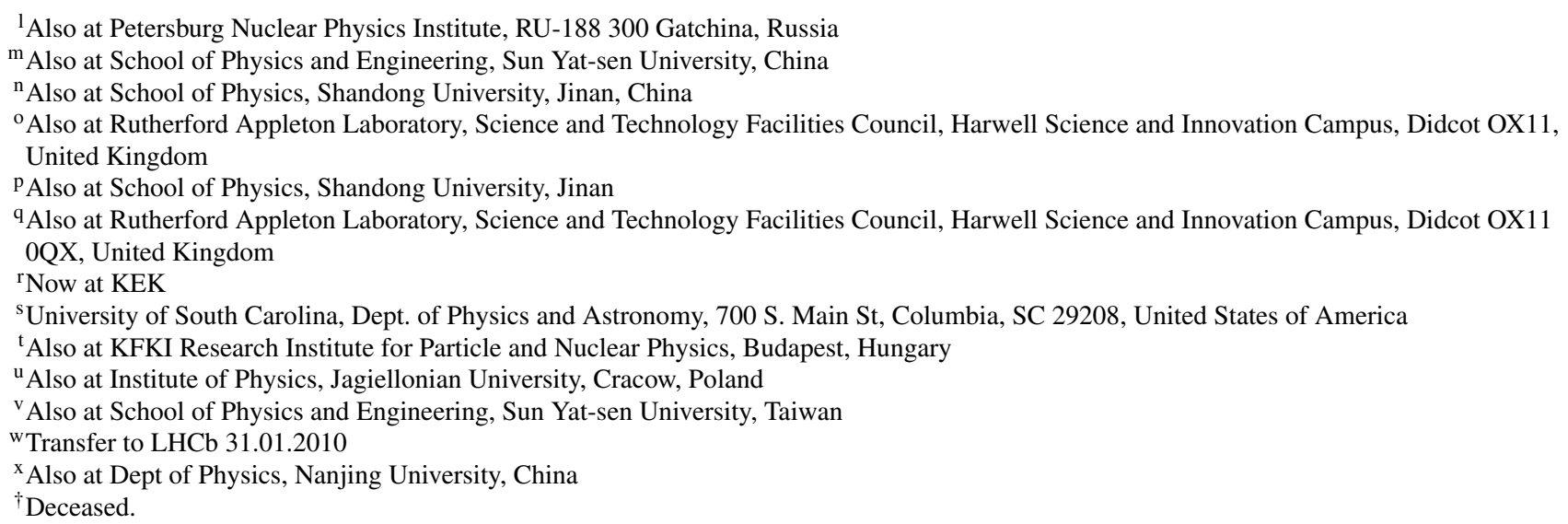

Received: 18 June 2010 / Revised: 19 July 2010 / Published online: 23 October 2010

(C) CERN for the benefit of the ATLAS collaboration 2010. This article is published with open access at Springerlink.com

\begin{abstract}
The ATLAS detector at the Large Hadron Collider has collected several hundred million cosmic ray events during 2008 and 2009. These data were used to commission the Muon Spectrometer and to study the performance of the trigger and tracking chambers, their alignment, the detector control system, the data acquisition and the analysis programs. We present the performance in the relevant parameters that determine the quality of the muon measurement. We discuss the single element efficiency, resolution and noise rates, the calibration method of the detector response and of the alignment system, the track reconstruction efficiency and the momentum measurement. The results show that the detector is close to the design performance and that the Muon Spectrometer is ready to detect muons produced in high energy proton-proton collisions.
\end{abstract}

\section{Contents}

1 The ATLAS Muon Spectrometer . . . . . . . . . 886

2 Data sample and reconstruction software . . . . 888

3 Trigger configuration during data taking . . . . . 890

4 Data quality assessment . . . . . . . . . . . 891

5 MDT chamber calibration ... . . . . . . . 894

6 Detector performance: efficiency and resolution . 897

7 MDT optical alignment . . . . . . . . . . . . . 902

8 Pattern recognition and segment reconstruction . 908

9 Track reconstruction . . . . . . . . . . . 910

10 Summary . . . . . . . . . . . . . . . . 914

Acknowledgements . . . . . . . . . . . . 915

Open Access . . . . . . . . . . . . . . . . . . . . . . 915

References ............... 915

${ }^{\star \star}$ e-mail: atlas.secretariat@ cern.ch

\section{The ATLAS Muon Spectrometer}

The ATLAS Muon Spectrometer (MS in the following) is designed to provide a standalone measurement of the muon momentum with an uncertainty in the transverse momentum varying from $3 \%$ at $100 \mathrm{GeV}$ to about $10 \%$ at $1 \mathrm{TeV}$, and to provide a trigger for muons with varying transverse momentum thresholds down to a few GeV. A detailed description of the muon spectrometer and of its expected performance can be found in [1-3]. Here only a brief overview is given. The muon momentum is determined by measuring the track curvature in a toroidal magnetic field. The muon trajectory is always normal to the main component of the magnetic field so that the transverse momentum resolution is roughly independent of $\eta$ over the whole acceptance. The magnetic field is provided by three toroids, one in the "barrel" $(|\eta|<1.1)$ and one for each "end-cap" $(1.1<|\eta|<2.7)$, with a field integral between 2 and $8 \mathrm{Tm}$. The muon curvature is measured by means of three precision chamber stations positioned along its trajectory. In order to meet the required precision each muon station should provide a measurement on the muon trajectory with an accuracy of $50 \mu \mathrm{m}$. In Fig. 1 a schematic view of the muon spectrometer ${ }^{1}$ is given.

For most of the acceptance Monitored Drift Tube (MDT) chambers are deployed [2]. The coordinate in the plane perpendicular to the wires, measured by the MDT, is referred to as the precision, or bending coordinate, being mainly per-

\footnotetext{
${ }^{1}$ The ATLAS reference system is a Cartesian right-handed coordinate system, with the nominal collision point at the origin. The positive $x$-axis is defined as pointing from the collision point to the center of the LHC ring and the positive $y$-axis points upwards while the $z$-axis is tangent to the beam direction at the collision point. The azimuthal angle $\phi$ is measured around the beam axis, and the polar angle $\theta$ is the angle measured with respect to the $z$-axis. The pseudorapidity is defined as $\eta=-\ln \tan \theta / 2$.
} 


\section{ATLAS}

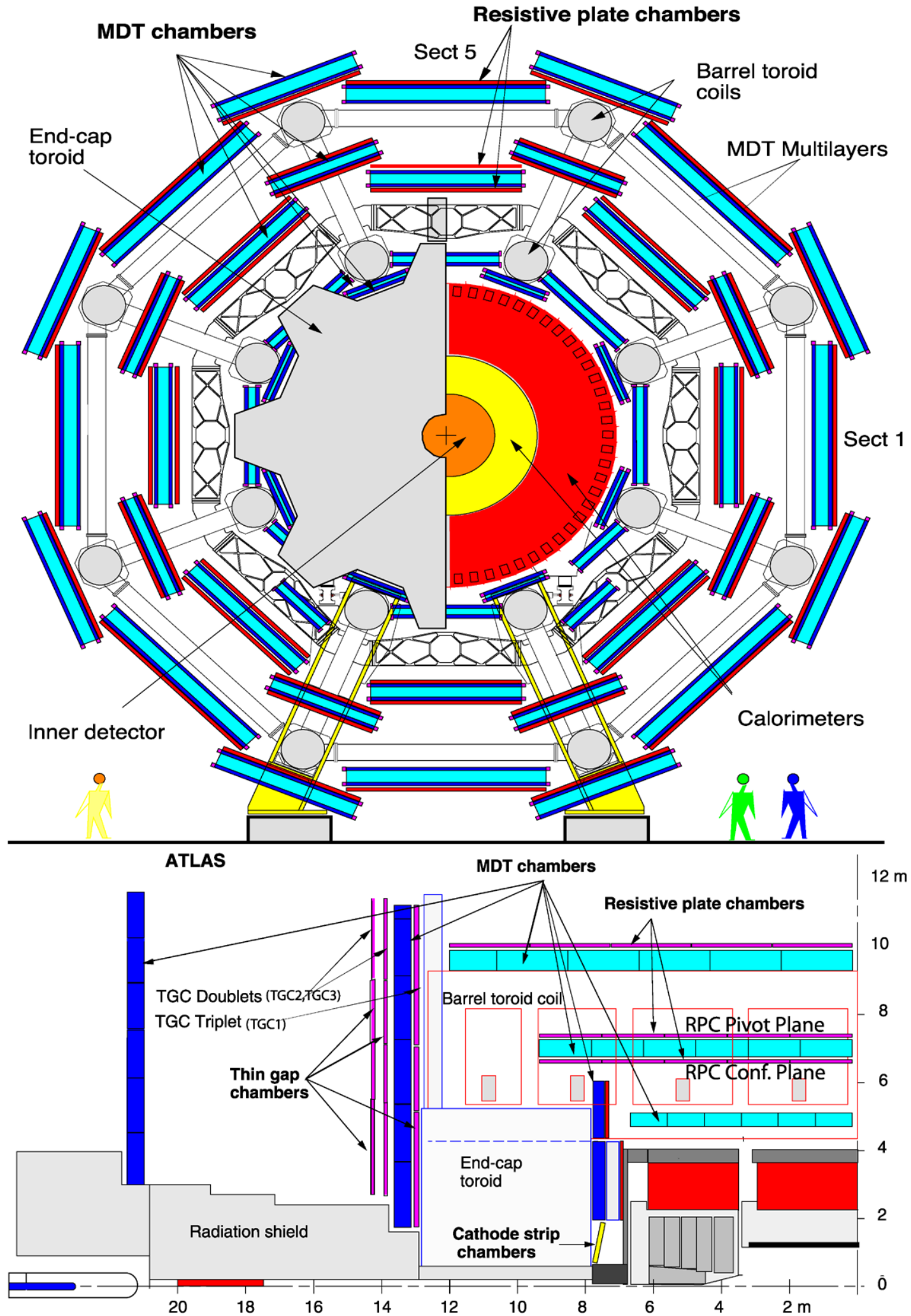

Fig. 1 Schematic view of the muon spectrometer in the $x-y$ (top) and $z-y$ (bottom) projections. Inner, Middle and Outer chamber stations are denoted BI, BM, BO in the barrel and EI, EM, EO in the end-cap 
pendicular to the direction of the toroidal field. In the endcap inner region, for $|\eta|<2.0$, Cathode Strip Chambers (CSC) [2] are used because of their capability to cope with higher background rates.

The MDT chambers are composed of two MultiLayers (ML) made of three or four layers of tubes. Each tube is $30 \mathrm{~mm}$ in diameter and has an anode wire of $50 \mu \mathrm{m}$ diameter. The gas mixture used is $93 \% \mathrm{Ar}$ and $7 \% \mathrm{CO}_{2}$ with a small admixture of water vapor, the drift velocity is not saturated and the total drift time is about $700 \mathrm{~ns}$. The space resolution attainable with a single tube is about $80 \mu \mathrm{m}$, measured in a test beam $[4,5]$. The CSC chambers are multiwire proportional chambers with cathode strip read out. The cathode planes are equipped with orthogonal strips and the precision coordinate is obtained measuring the charge induced on the strips making the charge interpolation between neighboring strips. Typical resolution obtained with this read-out scheme is about $50 \mu \mathrm{m}$.

The trigger system of the MS is based on two different chamber technologies: Resistive Plate Chambers (RPC) [2] instrument the barrel region while Thin Gap Chambers (TGC) [2] are used in the higher background environment of the end-cap regions. Two RPC chambers are attached to the middle barrel chambers providing a low- $p_{T}$ trigger. A high$p_{T}$ trigger is provided by the RPC modules installed on the outer barrel chambers in combination with the low $p_{T}$ signal provided by the middle chambers. The RPCs also provide the coordinate along the MDT wires that is not measured by the MDT chambers.

Similarly in the end-cap two TGC doublets and one triplet are installed close to the middle station and provide the low- $p_{T}$ and high- $p_{T}$ trigger signals. The TGCs also measure the coordinate of the muons in the direction parallel to the MDT wires. This coordinate is referred to as the second, or non-bending coordinate. For this purpose TGC chambers are also installed close to the MDTs in the inner layer of the end-cap (EI).

Some MS naming conventions adopted in this paper are introduced here. The MS is divided in the $x-y$-plane (also referred to as $\phi$-plane) in 16 sectors: Sector 5 being the upper most and Sector 13 the lower most. In both barrel and end-cap regions the MS is divided into 8 'Large' sectors (odd numbered sectors) and 8 'Small' sectors (even numbered sectors), determined by their coverage in $\phi$. The muon stations are named 'Inner', 'Middle', and 'Outer', according to the distance from the Interaction Point (IP). The three stations for the barrel are denoted $\mathrm{BI}, \mathrm{BM}$, and $\mathrm{BO}$, and for the End-Cap EI, EM, and EO, respectively. Along the $z$ axis, the MS is divided into two sides, called side A (positive $z$ ) and $\mathrm{C}$ (negative $z$ ).

As a complementary source of information, two publications $[4,5]$ on a detector system-test with a high momentum muon beam can be consulted.
Beginning in September 2008 the ATLAS detector was operated continuously up to November 2008 and then for different periods starting from Spring 2009. The first beams were circulated in the LHC machine in September 2008 but no beam-beam collisions were delivered. During these periods, the ATLAS detector collected mainly cosmic ray data. All muon detector technologies were included in the run with the exception of CSCs for which the Read Out chain was still not yet commissioned and therefore they are not included in the results presented in this paper.

The analyzed data samples and the reconstruction software are described in Sect. 2. The cosmic ray trigger is described in Sect. 3. Studies of data quality, calibration, and alignment are presented in Sects. 4, 5, 6, 7 respectively, while studies on tracking performance are presented in Sects. 8 and 9. The results are summarized in Sect. 10.

\section{Data sample and reconstruction software}

\subsection{Data sample}

In preparation for LHC collisions, the ATLAS detector has acquired several hundred million cosmic ray events during several run periods in 2008 and 2009. The analysis of a subset of data corresponding to about $60 \mathrm{M}$ events is presented here. These runs allowed commissioning the ATLAS experiment, the trigger, the data acquisition, the various detectors and the reconstruction software. Most of the cosmic rays reach the underground detectors via the two big shafts. They have incident angles close to the vertical axis and they are mainly triggered by the RPCs. The selected runs, together with the status of the magnetic field in the MS and the number of collected events for the different trigger streams, are listed in Table 1.

Table 1 List of analyzed data runs together with the corresponding trigger stream, statistics and status of the MS magnetic field. All runs were collected in Fall 2008, with the exceptions of run 113860 collected in Spring 2009, and run 121080 in Summer 2009

\begin{tabular}{lllll}
\hline Run & Trigger & B-field & $N$ of Evts & Period \\
\hline 91060 & RPC & Off & $17 \mathrm{M}$ & Fall 08 \\
91060 & TGC & Off & $0.2 \mathrm{M}$ & Fall 08 \\
89106 & TGC & Off & $0.4 \mathrm{M}$ & Fall 08 \\
89403 & TGC & Off & $0.4 \mathrm{M}$ & Fall 08 \\
91803 & TGC & On & $50 \mathrm{~K}$ & Fall 08 \\
91890 & RPC & On & $16 \mathrm{M}$ & Fall 08 \\
113860 & RPC & Off & $6 \mathrm{M}$ & Spring 09 \\
121080 & RPC & On & $21 \mathrm{M}$ & Summ 09 \\
\hline
\end{tabular}




\subsection{Muon reconstruction software}

The data were processed using the complete ATLAS software chain [6]: data decoding, data preparation (which includes calibration and alignment), and track reconstruction. Muon reconstruction has been handled by two independent packages, namely Moore [7] and Muonboy [8]. The two reconstruction algorithms are similar in design but differ in some details. The general strategy is to reconstruct muon trajectories both at the local (individual chamber), as well as at the global (spectrometer), level. The trajectories reconstructed in individual chambers can be approximated as straight lines over a short distance where bending has little effect and are therefore fit to track segments. Full tracks are formed by combining segments from multiple chambers.

Prompt muons produced in proton-proton collisions have trajectories that point back to the Interaction Point (IP). Moreover they are synchronous with the collision since all the detector front end electronics are synchronized with the LHC bunch crossing frequency of $40 \mathrm{MHz}$. In contrast, cosmic ray muons are "non-pointing" and are asynchronous with the detector clock: they have an additional $25 \mathrm{~ns}$ jitter with respect to the clock selected by the trigger. In addition, during commissioning the different trigger detectors were not timed with sufficient precision, leading to variations in timing depending on the region of the detector that originated the trigger. A further difficulty in track reconstruction was due to the lack of precise alignment of the muon detectors during this commissioning phase, as described in Sect. 7.

The reconstruction algorithms were adapted for these "cosmic ray" conditions as described below. Both programs were modified by relaxing the standard tracking requirements and implementing a procedure to accommodate the cosmic ray timing conditions. The tolerance for hit association to form track segments and the uncertainty associated with each hit position were increased. Moreover, a procedure called global $t_{0}$ refit ( $\mathrm{G} t_{0}$-refit) was developed in both reconstruction algorithms to compensate for the $25 \mathrm{~ns}$ time jitter and the imprecise trigger timing. The aim of this procedure is to determine with better precision the time when the cosmic ray crossed the detector by introducing a free global timing parameter $\left(\mathrm{g} t_{0}\right)$ in the segment reconstruction. The implementation of the $\mathrm{G} t_{0}$-refit in the two reconstruction algorithms is briefly described below while the results are presented in Sect. 5 .

\subsection{Muonboy track reconstruction}

The strategy of the Muonboy reconstruction algorithm can be summarized in four main steps:

- identification of Regions Of Activity (ROA) in the muon system with the information provided by the RPC/TGC detectors
- reconstruction of local segments in each muon station in the identified ROA

- combination of segments of different muon stations to form muon track candidates using three-dimensional tracking in magnetic field

- global track fit of the muon track candidates through the full system using individual hit information.

The topology of cosmic ray tracks is accommodated by relaxing the Region Of Activity requirement of pointing in a projective geometry when associating hits to form segments, or matching segments to form tracks. Moreover, since cosmic ray events have low occupancy, looser quality criteria were used for the selection of segments and tracks.

The Muonboy algorithm for the $\mathrm{G} t_{0}$-refit consists of a scan of different $g t_{0}$ values in steps of $10 \mathrm{~ns}$, doing the full segment reconstruction at each step. The $\mathrm{g} t_{0}$ value giving the best reconstruction quality factor is kept and a parabolic fit is performed using this best value and the two closer values along the parabola. Then the $\mathrm{g} t_{0}$ corresponding to the minimum of the quality factor parabola is chosen. In order to obtain high efficiency, the accuracy requirement for the MDT single hit resolution is relaxed by adding in quadrature a $0.5 \mathrm{~mm}$ constant smearing to the intrinsic resolution function (described in Sect. 6). This smearing is increased by additional $0.5 \mathrm{~mm}$ if the $\mathrm{G} t_{0}$-refit fails. Moreover, a less demanding track quality factor is required for tracks when hits are missing or are not associated to the track.

\subsection{Moore track reconstruction}

The Moore reconstruction algorithm is built out of several distinct stages:

- identification of global roads throughout the entire spectrometer using all muon detectors (MDT, CSC, RPC and TGC)

- reconstruction of local segments in each muon station seeded by the identified global roads

- combination of segments of different muon stations to form muon track candidates

- global, three-dimensional, tracking and final track fit.

Several modifications to the standard pattern recognition were made to optimize the reconstruction of cosmic ray tracks. In the global road finding step, a straight line Hough transform was used to allow for non-pointing tracks. The cuts on distance and direction between the road and the segment were relaxed. In the segment finding no cuts were applied on the number of missing hits (i.e. drift tubes that are expected to be crossed but have no hits).

The $\mathrm{G} t_{0}$-refit consists in varying simultaneously the global time offset $\left(\mathrm{g} t_{0}\right)$ for each segment reconstructed in a chamber. Then all measured times of hits associated to the segment are translated into drift radii after subtraction of the 
$\mathrm{g} t_{0}$. The $\mathrm{g} t_{0}$ value that minimizes the sum in quadrature of the weighted residuals (corresponding to the segment reconstruction $\chi^{2}$ ) is selected.

In this fit the MDT uncertainties are set to twice the testbeam drift tube resolution. If the segment fit is not successful, a straight line fit is performed assuming a constant $1 \mathrm{~mm}$ error. Hits are removed if their distance from the segment is greater than $7 \sigma$. In the track fit the MDT errors are enlarged to $2 \mathrm{~mm}$ to account for uncertainties in the alignment of chamber stations.

\section{Trigger configuration during data taking}

A more detailed description of the trigger system can be found in $[2,9]$. Here only specific issues related to the 2008-2009 cosmic ray data taking are introduced. The muon level-1 trigger is issued by the RPC in the barrel and by the TGC in the end-caps. During cosmic ray data taking most of the statistics were collected using this trigger. Special trigger configurations were adopted with different geometries (e.g. non pointing to the IP) and different timing (e.g. delaying the triggers issued by the upper sectors in order to trigger only in the lower sectors to mimic particles coming from the IP) when commissioning the muon trigger system itself or when selecting cosmic rays for commissioning the other ATLAS sub-systems.

In beam-collision configuration, the level-1 muon trigger selects pointing tracks with six different thresholds in transverse momentum and sends information to the CentralTrigger-Processor (CTP). The six thresholds, three low- $p_{T}$ and three high- $p_{T}$, do not distinguish between different detector regions, barrel or end-cap. For cosmic rays, to help commissioning separately the two regions, it was chosen to assign three thresholds to the barrel and three to the end-cap.

\subsection{Barrel level-1 trigger}

The barrel trigger detectors are arranged in three stations each having a doublet of RPC layers at increasing distances from the IP. In each sector the first two stations are mechanically coupled to the BM MDT while the third is coupled with the BO MDT as shown in Fig. 1.

The trigger algorithm is steered by signals on the middle layers, named Pivot plane. When a hit is found on this plane, the low- $p_{T}$ trigger logic searches for hits in the inner layers, named Confirm plane, and requires a coincidence in time of three hits over the four layers in a pre-calculated cone. The width of this cone defines the $p_{T}$ threshold. If hits are also found in a pre-calculated cone of the outermost plane in coincidence with a low- $p_{T}$ trigger, a high- $p_{T}$ trigger is issued. Also in this case the $p_{T}$ threshold is defined by the width of the cone. In addition to the $p_{T}$ requirement, the trigger logic also demands the track to be pointing towards the IP both in $\phi$ and $\eta$. In the cosmic ray runs only three of the six thresholds were used in the barrel and were defined as MUO_LOW, MUO_HIGH and MU6. The two thresholds MUO_LOW/HIGH did not select a physical $p_{T}$ range; in fact, the MUO_LOW was triggered only by the time coincidence of 3 out of 4 hits without any pointing constraint and the MUO_HIGH was triggered by the coincidence of a MUO_LOW with at least a hit in the corresponding outer plane. The threshold MU6 required not only a time coincidence but also an IP-pointing constraint in the $\phi$-projection only. To emulate the timing expected for beam collisions, and to enhance the illumination of the Inner Detector (ID), the cosmic ray trigger was issued mainly by the bottom sectors. This was achieved by delaying the top sector trigger by $5 \mathrm{BC}$ (125 ns) preventing it from arriving first at the Central Trigger Processor (CTP) and thus forming the trigger.

In the fall 2008 data taking period, the timing of the low$p_{T}$ trigger and the data read-out latencies were still under commissioning. This had a large impact on the detector coverage. The situation has largely improved for the runs taken in 2009 both in terms of detector coverage and in trigger timing as shown in Sects. 4.3 and 6.2.

\subsection{End-cap level-1 trigger}

The level-1 TGC trigger system provided three thresholds, named MUO_TGC_HALO,MUO_TGC and MU6_TGC. The trigger was issued by the coincidence between several TGC layers. The logic was based both on timing (BC identification) and geometry (pointing track). The main difference between the three trigger thresholds is related to the required number of layers and to the degree of pointing to the IP. MUO_TGC_HALO required a 3 out of 4 layer coincidence in the two outermost TGC stations, the so-called Doublet chambers, in both $\eta$ (bending) and $\phi$ (non-bending) projections and a pointing requirement within $20^{\circ}$. MUO_TGC and MU6_TGC required in addition a 2 out of 3 layer coincidence in the TGC stations closer to the IP, the so-called Triplet chambers, in the $\eta$ projection only. The pointing requirement of MUO_TGC was of $\pm 10^{\circ}$ degrees while for MU6_TGC was of $\pm 5^{\circ}$.

The trigger was timed for high-momentum muons coming from the IP. All the delays due to different time-of-flight and cable lengths were properly set and cross-checked using a test pulse system achieving a relative timing within $4 \mathrm{~ns}$. For most of the cosmic run period, only the level-1 trigger generated from the TGC bottom sectors was used. This was chosen to ensure good timing of the trigger with the read-out of the ID, since cosmic muons triggered by the TGC bottom sectors and crossing the ID have a time-of-flight similar to muons produced in collisions. 


\section{Data quality assessment}

\subsection{Introduction}

The data quality assessment consists of several software algorithms working at different levels of the data taking. The Detector Control System (DCS) [11] is the first source of information available during the operation of the detector. Here information on the hardware status of the different subdetectors and on the settings of Low Voltage (LV) and High Voltage (HV) power supplies and on the gas system is available. The DCS also receives information from the Data Acquisition (DAQ) [11] as soon as problems during the readout of a chamber appear.

The next stage in the chain of data quality assessment is the on-line monitoring. It receives input from the data acquisition system running in a spectator mode. Once the data are decoded, monitoring histograms are filled showing quantities related to the detector operation. Part of the muon data selected by the level-1 trigger Region Of Interest (ROI) are transferred by the level-2 trigger processors to three dedicated computing farms (referred to as calibration centers) to monitor and determine the calibration parameters of the MS chambers. The larger event samples available at the calibration centers allow the analysis of single drift tube responses. The goal of the analysis at the calibration centers is to provide drift tube and trigger chambers calibration constants and to give general feedback on the detector operation within 24 hours, which is the time needed, at high luminosity, to collect enough statistics to calculate new calibration constants.

On a longer time scale, using the full reconstructed ATLAS event information, the off-line data monitoring provides the final information on the data quality. At each step a flag summarizing the data quality at that level is stored in a database.

\subsection{MDT chambers}

In the fall 2008 period (e.g. Run 91060) only five out of 1110 MDT chambers were not included in the data taking. Of these five chambers, two were not yet connected to services and three had problems with the gas system. Due to the cosmic ray illumination and the trigger coverage not all chambers had sufficient event samples to determine the performance of single drift tubes. The studies reported here were done at different levels of detail, from chamber information down to single drift tube information when the event samples were sufficient. The data survey searched for problems of individual read-out channels as well as of clusters corresponding to hardware related groups of tubes. A screen shot of one of the online monitoring applications used for the MDT chambers is shown in Fig. 2. Here the average number of hits per tube for each MDT is represented in a $\eta-\phi$ plot where the higher cosmic illumination on the top and bottom sectors $(3-7,11-15)$ compared to the vertical sectors (16-2 and 8-10) is clearly seen as well as the larger illumination on the A side of the detector where the larger shaft is present. The five chambers not included in the data acquisition are marked as dark gray boxes. Two more chambers are visible with very low statistics due to problems with the HV supplies. For 32 MDT chambers one of the two multi-layers was disconnected from HV.

A detailed list of hardware problems found in run 91060 is reported in Table 2. The cosmic ray flux was not sufficient for a detailed analysis of single drift tubes for 15 MDT chambers ( $\sim 3 \mathrm{~K}$ channels). Thus we were able to analyze individually $336 \mathrm{~K}$, out of the working $339 \mathrm{~K}$, drift tubes. To summarize, about $5 \mathrm{~K}$ channels, out of $336 \mathrm{~K}$, have shown some problems in run 91,060 , corresponding to $1.5 \%$. Most of these channels have been recovered during the 2008-2009 shutdown period. Only a very small fraction of problems, at the level of a few per mill, could not be solved, such as permanently disconnected tubes (broken wires) or chambers with very difficult access.

In addition to monitoring in the DAQ framework (online monitoring), the data are also processed with the offline reconstruction program which produces monitoring histograms. This ensures that the reconstruction works properly and that the correct conditions data (calibration and alignment constants) are used in the first processing of the data. The off-line monitoring gathers and presents information on several variables for single drift tubes, e.g. drift time and collected charge distributions, hit occupancy and noise rate. These variables are obtained for individual MDTs or grouped for regions, such as $\eta$ or $\phi$ sectors, barrel or endcap, side A or C. Variables related to segments or tracks are also monitored.

\subsection{Barrel trigger chambers: RPC}

Commissioning of the RPC detectors progressed continuously and substantial improvements were made during the 2008-2009 shut-down. As an example Fig. 3 shows a twodimensional distribution of RPC strips requiring a 3 out of 4 majority coincidence for the low- $p_{T}$ trigger demonstrating that the trigger coverage in Spring 2009 was at the $95 \%$ level.

Studies of the trigger performance were made using the data of run 91,060 after implementation of the trigger roads [10]. For the low- $p_{T}$ trigger the four RPC layers in the Middle station are involved, both in $\eta$ and $\phi$ projections. Tracks were accepted by the trigger if any strip of the pivot plane was in coincidence with a group of strips of the confirm plane aligned with the IP, realizing a majority combination of 3 out of 4 RPC layers. Figure 4(A) shows the spatial 


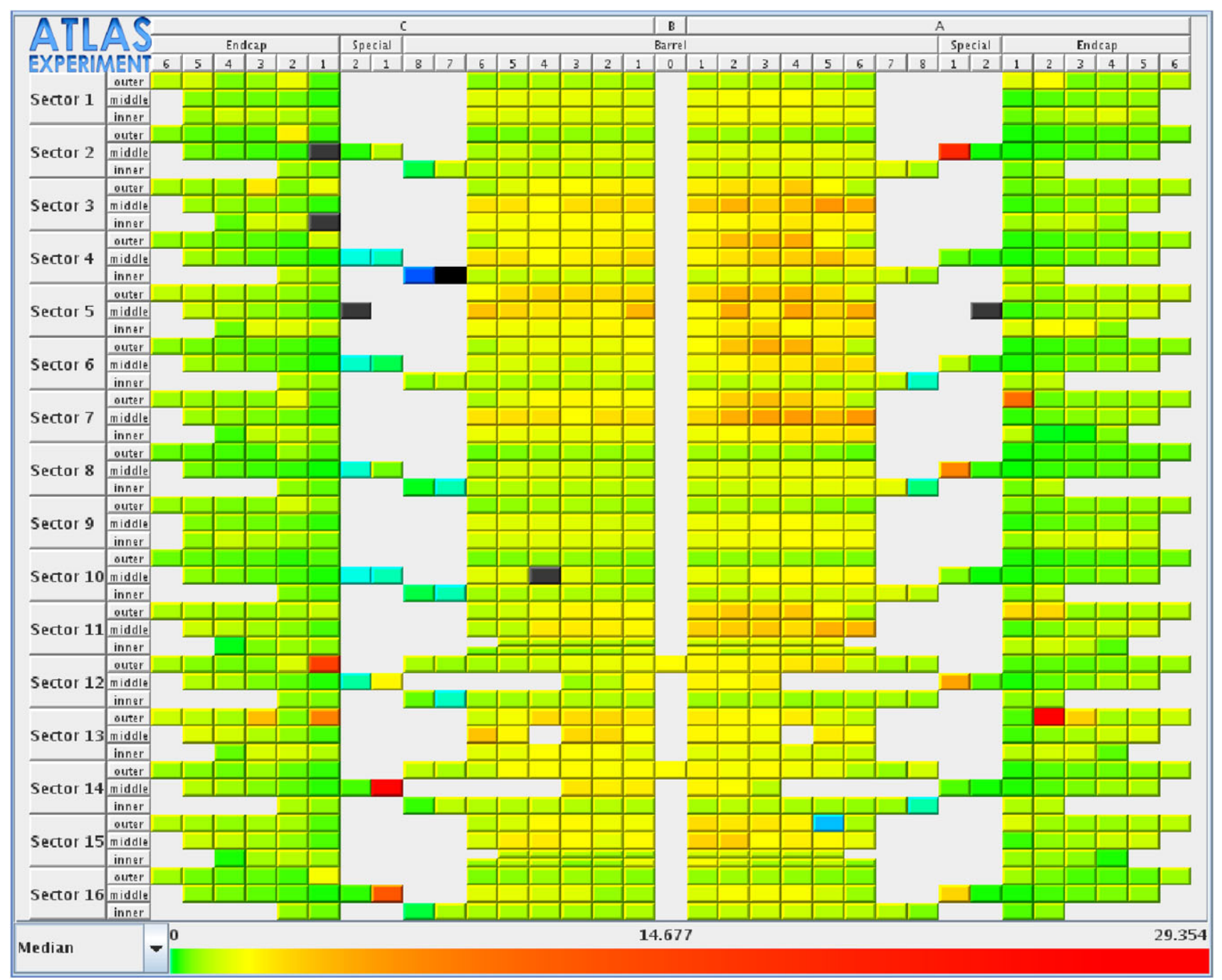

Fig. 2 Screen shot of a monitoring application displaying the MDT hit occupancy for all chambers. Each chamber is represented by a small box. The color of the box is related to the average number of raw hits per tube. The boxes are arranged in an $\eta-\phi$ grid: a column represents an $\eta$ slice, perpendicular to the beam axis; a row represents one of the sixteen $\phi$ sectors. Within each sector chambers of the Inner, Middle, Outer ring are displayed separately
Table 2 List of MDT channels with problems in run 91,060

\begin{tabular}{lcc}
\hline Number of channels analyzed with sufficient event samples & 336,144 & Fraction \\
\hline Channels not included in the read-out & 936 & $0.28 \%$ \\
Channels with read-out or initialization problems & 744 & $0.22 \%$ \\
Channels with HV or gas problems & 2942 & $0.88 \%$ \\
Permanently dead channels (broken wires) & 323 & $0.10 \%$ \\
Total problematic channels & 4945 & $1.47 \%$ \\
\hline
\end{tabular}

correlation between $\phi$ strips in the pivot planes and $\phi$ strips in the confirm planes. The correlation line is slightly rotated with respect to the diagonal due to the different distance of the confirm and pivot planes with respect to the IP.

A random trigger was used to measure the counting rate for each read-out strip. This is a measurement of the RPC system noise rate. About $310 \mathrm{~K}$ strips were analyzed over a total of $350 \mathrm{~K}$ working strips. Figure 4(B) shows the distribution of single channel noise rate, normalized to an area of $1 \mathrm{~cm}^{2}$. For each strip, the noise rate is calculated as the number of hits divided by the number of random triggers and the width of the read-out gate of $200 \mathrm{~ns}$, and is normal- 


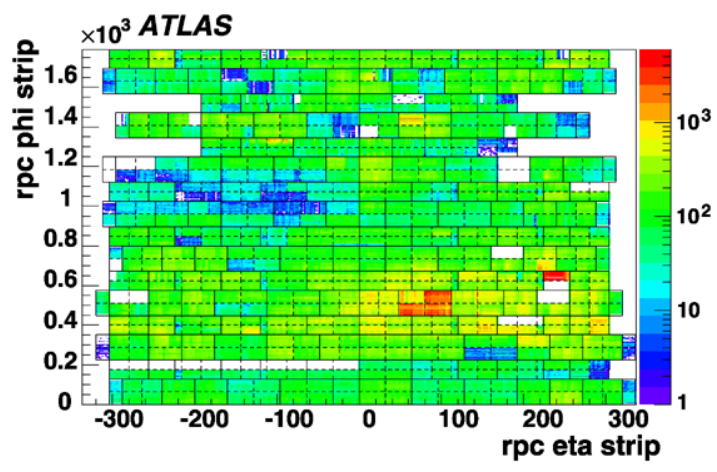

Fig. 3 RPC low- $p_{T}$ trigger coverage in $\eta-\phi$ for Run 113,860 (Spring 2009). Each $\eta$ and $\phi$ strip producing a low- $p_{T}$ trigger corresponds to an entry in the plot. The coverage in Spring 2009 was about $95 \%$
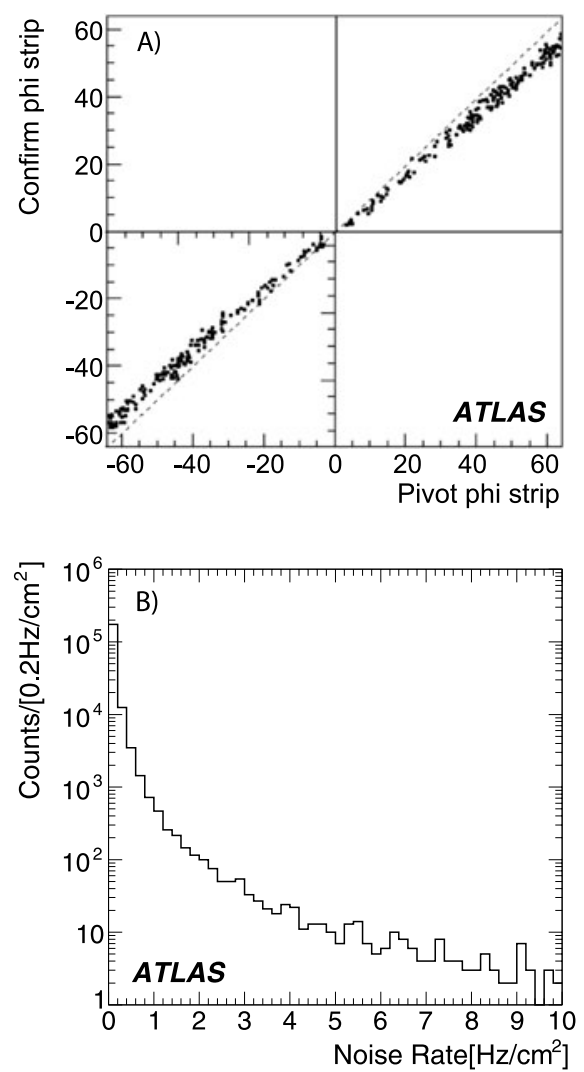

Fig. 4 (A): RPC spatial correlation between the pivot strip number and the confirm strip number in the $\phi$ projection for a programmed trigger road. 128 strips correspond to a RPC plane $3.8 \mathrm{~m}$ long. (B): Distribution of strip noise rates per unit area measured with a random trigger for $310 \mathrm{~K}$ RPC strips. The larger noise present on some strips is probably due to local weaknesses of grounding connections

ized to the area of the strip (typically $550 \mathrm{~cm}^{2}$ for a BM eta strips and $900 \mathrm{~cm}^{2}$ for a BO eta strips). Only a few hundred strips showed a counting rate above $10 \mathrm{~Hz} / \mathrm{cm}^{2}$ which is the background rate expected when the LHC will run at highluminosity. The average noise rate of the RPC was stable during the different running periods.
The fraction of dead channels, considering only the part of the detector included in the read-out in the Fall 2008 runs, was $1.5 \%$, mainly due to problems in the front-end electronics.

\subsection{End-cap trigger chambers: TGC}

In the end-caps the muon trigger is provided by the TGC chambers installed in three layers that surround the MDT Middle chambers. All together they form the so-called Big Wheels (BW), one in each end-cap. In addition, TGC chambers are also installed close to the EI chambers in the Small Wheels (SW), but these are only used to measure the muon $\phi$ coordinate. In Fall 2008 all the BW TGC sectors were readout. Given the installation schedule for the ATLAS detectors, the Inner TGC station were the last chambers installed and they were not fully operational during 2008 runs. For this reason they are not discussed in the following.

Two types of trigger configuration were adopted in Fall 2008. One was optimized to study the end-cap muon detectors with cosmic rays. In this configuration all TGC BW sectors were used in the trigger. The other setting was optimized to provide the trigger for the ID tracking detectors and was used for timing the ID. In order to mimic muons coming from the IP, only the five bottom sectors were used to trigger. The typical detector coverage in these two trigger configurations is shown in Fig. 5 by plotting the coincidence positions in the $x-y$ plane for wire and strip hits for run 91,060 (A) and run 91,803 (B). Only about $0.8 \%$ of chambers were not operational due to $\mathrm{HV}$ or gas problems. Since for the trigger a majority logic is required these inactive chambers do not produce any dead regions in the trigger acceptance.

The HV and front-end threshold setting, the gate widths for wires and strips, and the trigger sectors are listed in Table 3 for these two runs.

For each trigger issued by the CTP, the TGC Read Out Driver (ROD) sends to the DAQ system the data corresponding to three Bunch Crossings (Previous, Current and Next BC) contained in two separate buffers. Of the two buffers, one is located in the front-end board where the wires and strips providing the low- $p_{T}$ coincidence are separately recorded. In the second buffer, located in the Sector Logic Board in the service counting room, the coincidence of the wire and strip signals is done. Each buffer has a programmable identifier that has to be adjusted in order to read out the correct (Current) BC data. Figure 6 shows the readout timing for the front-end and the sector logic buffers for level-1 triggers issued by the TGC. About $98.6 \%$ of data in the front-end buffer, and $99.8 \%$ of data in the sector logic buffer are read out with the correct timing. The small population in the previous or next $\mathrm{BC}$ is due to cosmic ray showers. 

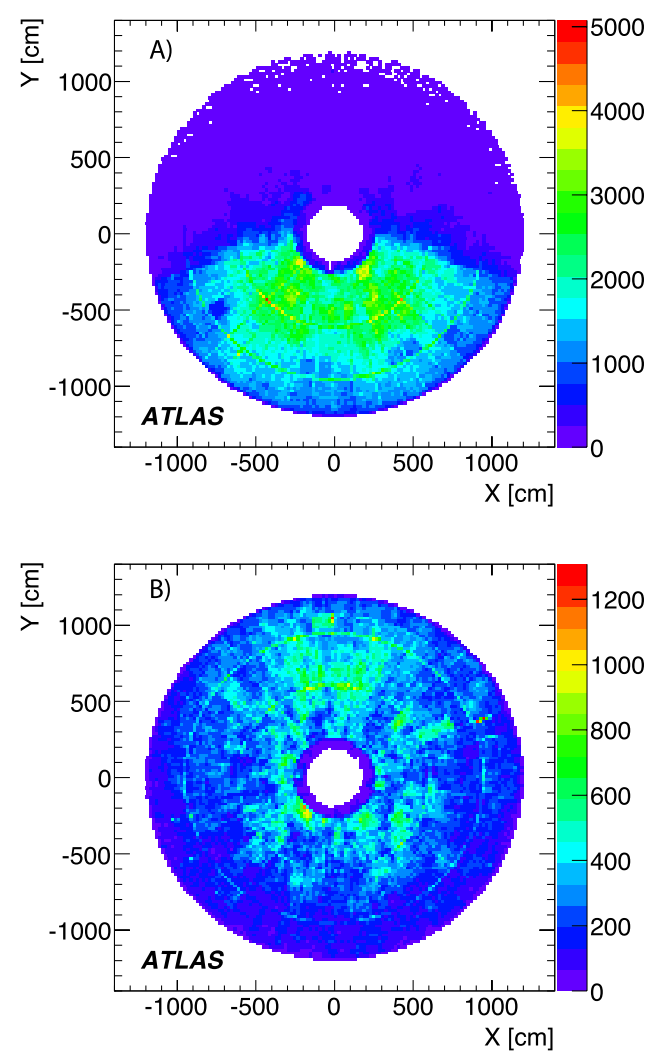

Fig. 5 Map of coincidences of wire and strip hits in the $x-y$ plane. (A): The five bottom sectors (sectors $8-12,195^{\circ}<\phi<345^{\circ}$ ) used for timing the ID tracking detectors in run 91,060. (B): With all sectors participating in the trigger during run 91,803

Table 3 TGC sectors participating in the trigger, high voltage setting, threshold and gate width

\begin{tabular}{lllll}
\hline Run & Trigger sector & HV & Threshold & $\begin{array}{l}\text { Gate widths } \\
\text { for wire/strip }\end{array}$ \\
\hline 91,060 & 8 to 12 & $2800 \mathrm{~V}$ & $100 \mathrm{mV}$ & $35 / 45 \mathrm{~ns}$ \\
91,803 & 1 to 12 & $2650 \mathrm{~V}$ & $80 \mathrm{mV}$ & $35 / 45 \mathrm{~ns}$ \\
\hline
\end{tabular}

\section{MDT chamber calibration}

\subsection{Calibration method}

The MDTs require a calibration procedure [12] to precisely convert the measured drift time into a drift distances from the anode wire (drift radius) that is subsequently used in pattern recognition and track fitting. The calibration of the MDT chambers is performed in three steps. In the first step the time offset with respect to the trigger signal, $t_{0}$, for each tube or group of tubes is determined; in the second step the drift-time to space relation, $r(t)$ function, is computed; in the third step the spatial resolution is determined.

The calibration constants are loaded in the Conditions Data Base (known as 'COOL') [13] and then retrieved, ac-
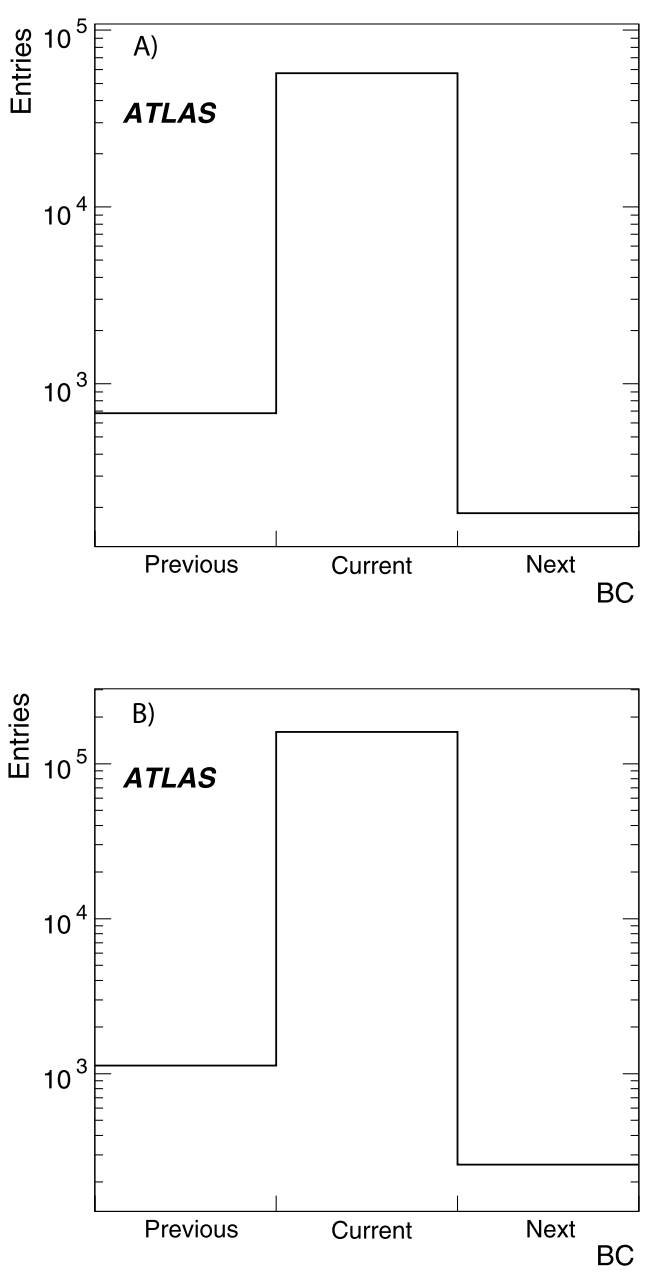

Fig. 6 (A): TGC front-end and (B): sector logic buffers for BC identification. Three $\mathrm{BC}$ crossing, previous, current and next are readout

cording to an Interval Of Validity (IOV) mechanism, to be used in the offline reconstruction. The IOV determines for which group of runs the calibration constants are valid. The gas mixture composition varied during the data taking period since the water injection part of the gas system was under commissioning resulting in a not constant admixture of water vapor, as can be seen in Fig. 9. Nonetheless the calibration procedure based on the IOV mechanism was able to provide good calibration constants for all the running period.

The $t_{0}$ offset depends on many fixed delays like cable lengths, front-end electronics response, Level-1 trigger latency, time of flight from the IP and has to be determined for each drift tube. The offset is obtained by fitting a Fermi function to the leading edge of the drift time distribution as shown in Fig. 7(A). The precision expected in LHC collision data is better than $1 \mathrm{~ns}$ with a dataset of about $10 \mathrm{~K}$ muons crossing the drift tube. This uncertainty does not significantly degrade the position resolution of the MDT tubes which corresponds to a time span of about 5 ns. In Fig. 7(B) also the typical spectrum of ADC for all tubes in a cham- 
ber is reported. Charge information in each tube is obtained using a Wilkinson ADC [14]. As the MDT chambers are operated at different temperatures depending on their positions in the MS, the $r(t)$ functions differ depending on location and are determined separately. In addition, variations of the toroidal magnetic field along the drift tubes produce different Lorentz angles, thus different $r(t)$ functions. An initial rough estimate of the $r(t)$ function is obtained with an accuracy of $0.5 \mathrm{~mm}$ by integrating the drift-time distribution. This is correct under the approximation of a uniform $d n / d r$ distribution, where $n$ is the number of hits at a drift radius $r$

$$
\frac{d n}{d t}=\frac{d n}{d r} \frac{d r}{d t}=\frac{N_{\mathrm{hits}}}{r_{\max }} \frac{d r}{d t} \Rightarrow r(t)=\frac{r_{\mathrm{max}}}{N_{\mathrm{hits}}} \int_{0}^{t} \frac{d n}{d t^{\prime}} d t^{\prime}
$$

$N_{\text {hits }}$ is the total number of hits in the time spectrum and $r_{\max }$ is the maximum drift radius $(14.4 \mathrm{~mm})$. In cosmic rays this approximation is only good at the level of a few hundred $\mu \mathrm{m}$ mainly because of the production of $\delta$-ray electrons along the track. An $r(t)$ relation with a higher accuracy, of about $20 \mu \mathrm{m}$, is obtained from this initial estimate by applying corrections, $\delta r(t)$, which minimize the
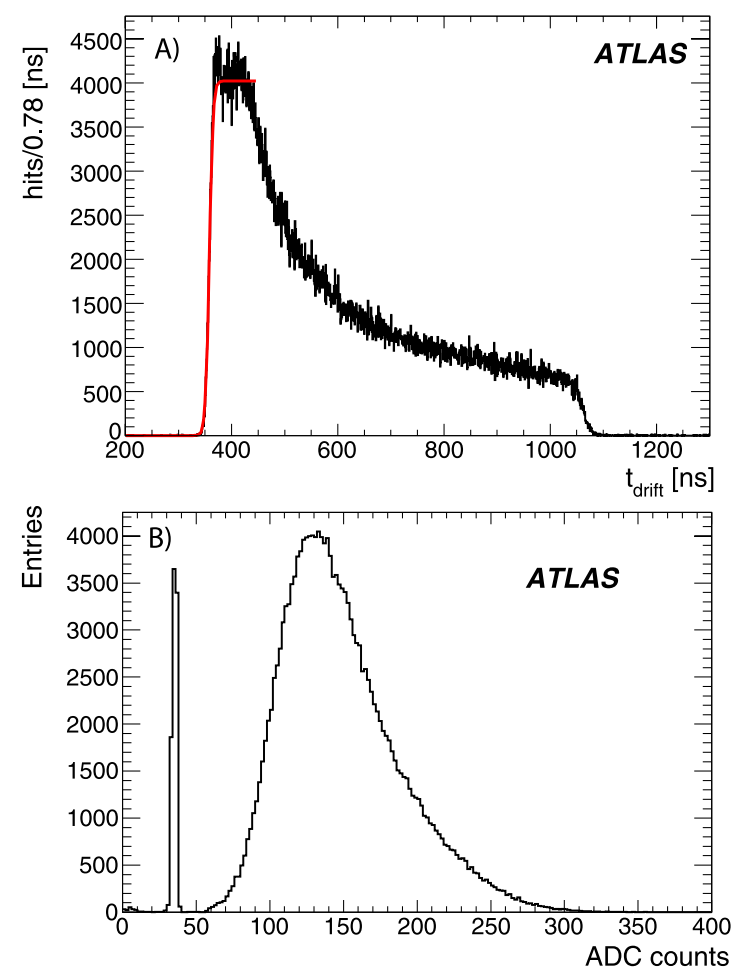

Fig. 7 (A): typical drift time spectrum in cosmic ray events for an MDT chamber. The position of the inflection point of the leading edge of the spectrum, $t_{0}$, is determined by fitting a Fermi function (shown in red) to the beginning of the spectrum. (B): Typical spectrum of ADC for all tubes in a chamber. Hits below 50 ADC counts are identified as electronic noise residuals of track segment fits with an iterative procedure. This minimization procedure, called auto-calibration, takes into account the dependence of the parameters of the fitted segments on the applied corrections $\delta r(t)$ and is mainly based on the geometrical constraints from the precise knowledge of the wire positions. Figure 8 shows a typical residual distribution of a chamber, as a function of the distance of the track segment from the anode wire, after the autocalibration.

In cosmic ray events additional sources of time jitter, beyond the intrinsic resolution, spoil the MDT measurement. The first cause of time jitter is due to cosmic ray muons crossing the tubes with an arbitrary phase with re-
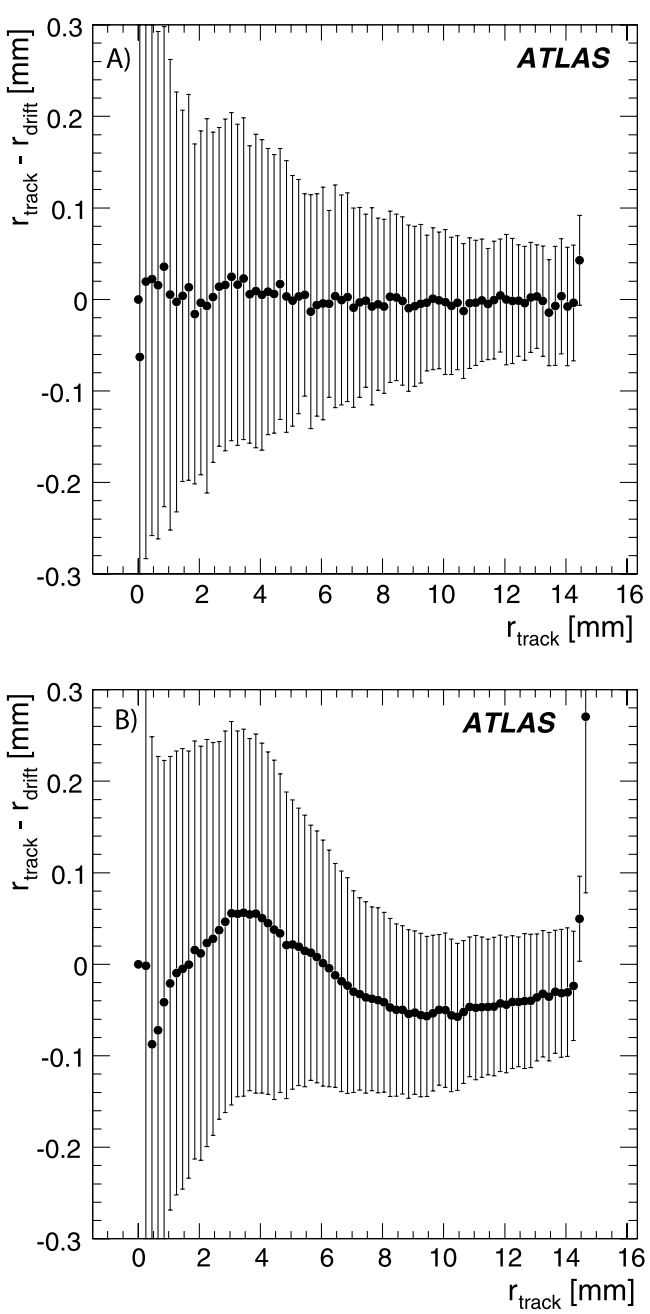

Fig. 8 (A): Residuals as a function of the track segment distance from the wire after the $r(t)$ auto-calibration and RPC-time corrections. The points correspond to the mean value of the distribution of residuals and the error bars to its RMS value. (B): Residuals as a function of the track segment distance from the wire after the $r(t)$ auto-calibration using the $\mathrm{G} t_{0}$-refit method. The points correspond to the mean value of the distribution of residuals and the error bars to its RMS value. Residual systematics at the level of $50 \mu \mathrm{m}$ are present using this correction 
spect to the front-end electronics clock [15]. This implies a time jitter corresponding to a 25 ns uniform distribution. The second cause is related to the spread of the trigger time for triggers generated in different parts of the detector (up to about $100 \mathrm{~ns}$ due to the initial stage of the trigger timing). Two different methods have been alternatively used to reduce the impact of these effects: the $R P C$ time correction and the MDT Gt $t_{0}$-refit. The achieved performance with both methods are discussed in Sect. 6. In the following a brief description of the former method is given.

The RPC-time correction uses the trigger time measured by the RPC chambers on an event by event basis. This time correction was applied only to the MDT chambers of the BM stations since these chambers are close to the two RPC stations used to issue the trigger and so no corrections due to time of flight and, more importantly, no corrections due to the spread in timing of the trigger signals issued by different parts of the detector are needed. This method cannot be applied to the end-cap region since the TGC do not provide a measurement of the trigger time but rather they select the appropriate BC.

With this correction the time jitter due to the two effects mentioned above is reduced from $\sim 100$ ns to few ns (see Sect. 6). The distribution of the residuals obtained after calibration using the RPC-time correction method is presented in Fig. 8(A). The precision of the auto-calibration is better than $\sim 20 \mu \mathrm{m}$ using this correction.

The $\mathrm{G} t_{0}$-refit has also been used to improve the single tube resolution, as discussed in Sect. 6. Also the precision of the auto-calibration is much improved with respect to the uncorrected situation. As shown in Fig. 8 a precision of $\sim 50 \mu \mathrm{m}$ is obtained for the residuals of the segment fit after auto-calibration with small residual systematics on the auto-calibration.

\subsection{End-cap chambers calibration with monitoring chamber}

For the end-cap MDTsystem, due to the limited number of cosmic ray events, a different method to determine the $r(t)$ relation was used. A small MDT chamber installed on the surface of the ATLAS underground hall was set up [16] to monitor continuously the MDT gas composition. One multilayer is connected to the supply line of the gas recycling system while the other is connected to the return line. This chamber benefits from a very large cosmic ray rate and can therefore determine the $r(t)$ function with high precision in short time intervals. Cosmic ray muons are triggered by scintillator counters mounted on the monitoring chamber. The trigger time is measured and subtracted event-by-event from the tube drift times, in this way the jitter related to the asynchronous front-end clock is automatically removed.

Data from the monitoring chamber are used to derive a $r(t)$ function every 6 hours to monitor the gas drift properties. Figure 9 shows the variation of the maximum drift time (the drift time of muons crossing the drift tube close to its edge) over the period September-October 2008. Two $r(t)$ functions were used to cover the Fall 2008 run period, for each period the $r(t)$ function for each chamber of the MS was corrected to account for the temperature difference using the data measured by the sensors mounted on any particular chamber. The temperature corrections to the $r(t)$ function were derived from the Garfield-MagBoltz simulation program [17-19]. The output of the simulation was validated by several measurements with a muon beam [5]. In the endcap region, the temperature varies by about $4^{\circ} \mathrm{C}$ from top to bottom of the MS, resulting in a variation of the maximum drift time of about $10 \mathrm{~ns}$. On the other hand the temperature of the cavern was remarkably stable in time.
Fig. 9 Maximum drift time measured by the gas monitor chamber versus time during September-October 2008. The red points refers to the return line and the blue points to the supply line (green and light blue points are the time average of the supply and return line measurements). The large variation seen between middle of September and 10th of October is due to the change of the quantity of water vapor added to the standard mixture

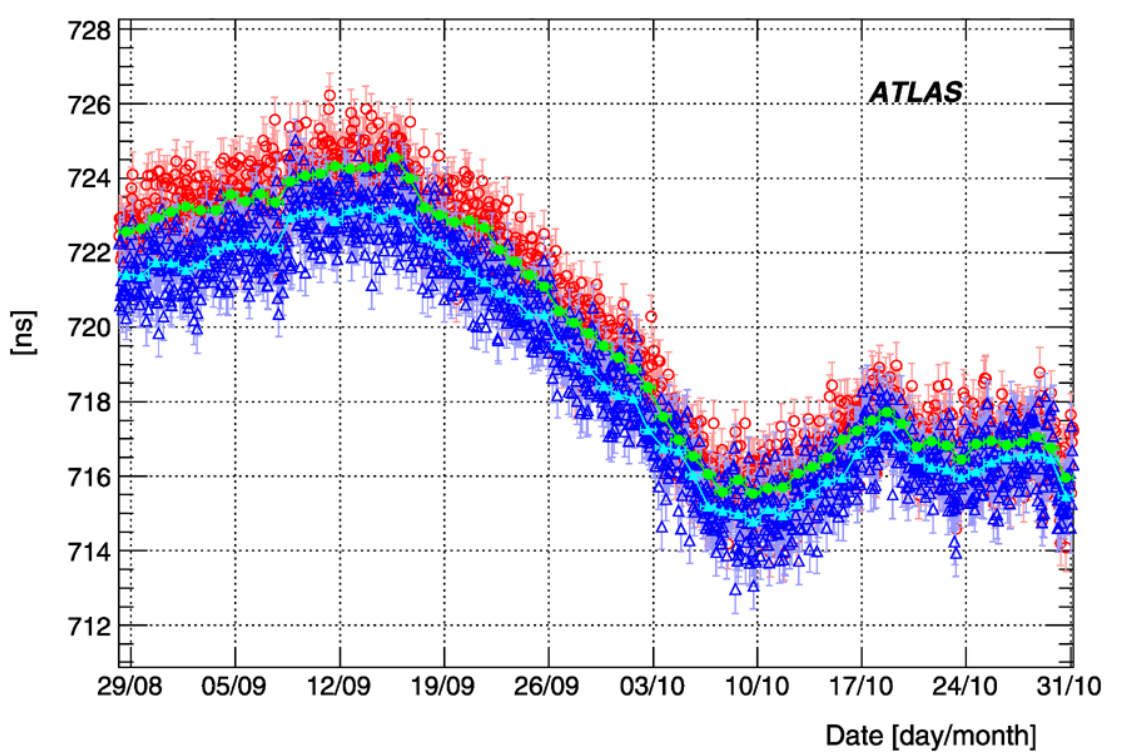


Fig. 10 (A) Distribution of the RMS and (B) of the mean values of the residuals from the fit to track segments in 373 end-cap chambers using the $r(t)$ function derived from the gas monitoring chamber. The black lines represent Gaussian fits to the distributions
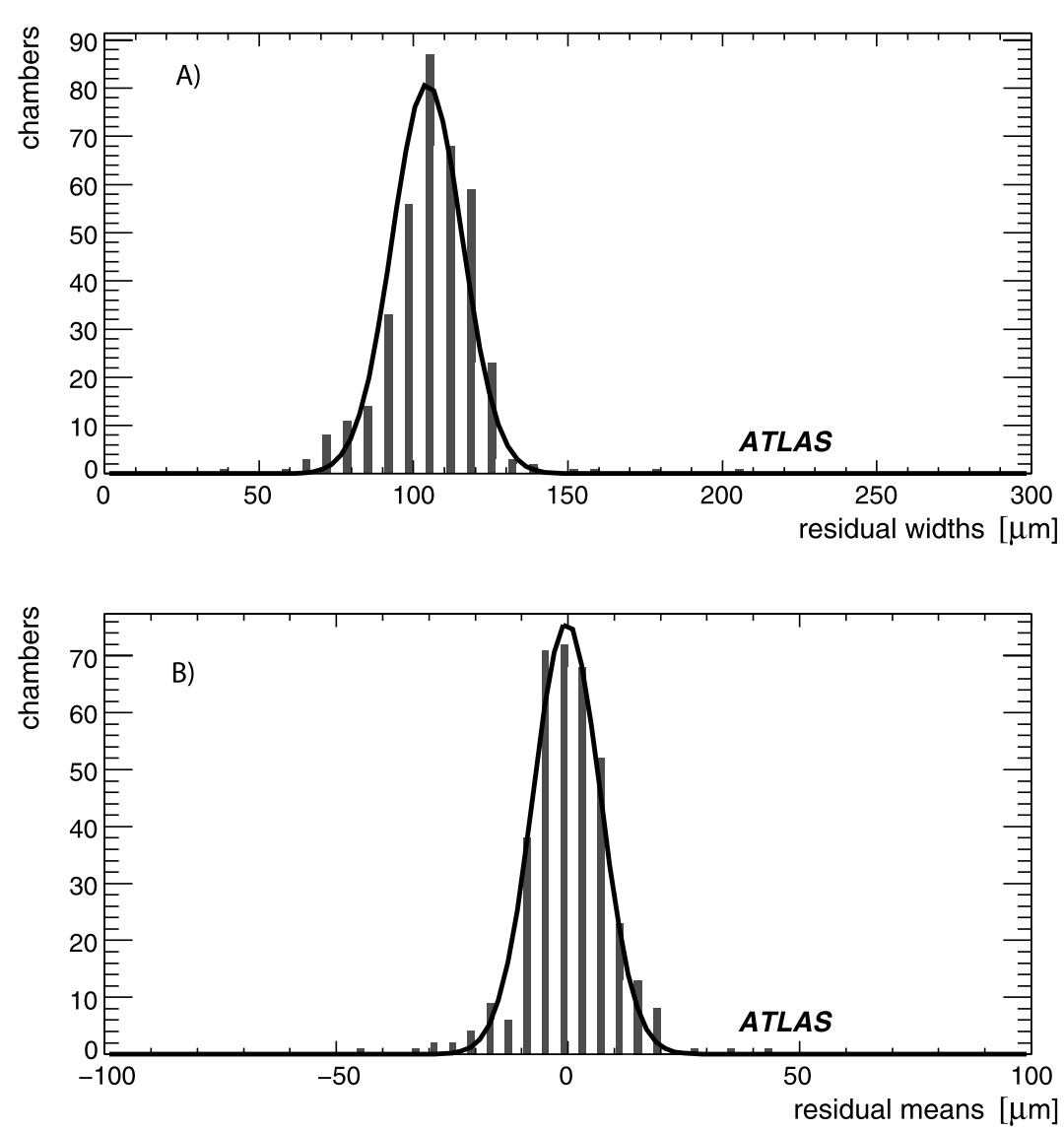

Figure 10 shows the distribution of the mean and RMS value of the residuals from the fit to track segments in all end-cap chambers (run 91,060). A Gaussian fit is superimposed. The $r(t)$ function derived from the gas monitor chamber with temperature corrections provides an acceptable calibration for all the MDT chambers of the endcap: the average standard deviation of the residuals is about $100 \mu \mathrm{m}$.

\section{Detector performance: efficiency and resolution}

\subsection{MDT}

\subsubsection{MDT drift time distribution}

The behavior of the drift time distributions of individual tubes is an important quality criterion for the MDT performance. The minimum and maximum drift times, $t_{0}$ and $t_{\max }$, respectively, correspond to particles passing very close to the wire and close to the tube walls, and their stability indicates the stability of the calibration. The number of hits recorded in a small time window before the rising edge of the drift time distribution $t_{0}$ can be used to estimate the rate of noise due to hits not correlated with the trigger. A precise knowledge of $t_{0}$ for each tube is essential for high quality segment and track reconstruction. As explained in Sect. 5, for cosmic rays some additional time jitter is present and must be accounted for.

In order to improve the quality of track reconstruction the $\mathrm{G} t_{0}$-refit time correction has been used. The performance of the $\mathrm{G} t_{0}$-refit algorithm has been investigated in the past, both using simulated data and using data taken with a BIL (Barrel Inner Large) chamber in a cosmic ray test stand under controlled trigger conditions [20]. The achieved $\mathrm{G} t_{0}$ resolution ranged between 2 and 4 ns depending on the chamber geometry ( 8 layer chambers have better resolution than 6 layer chambers) and hit topology. In particular the $\mathrm{G} t_{0}$-refit algorithm cannot work if all the hits are on the same side of the wires, typically for tracks at $30^{\circ}$ with respect to the chamber plane. The selection of good quality segments requires a minimum of five MDT hits and segments with all hits on the same side of the wires are removed.

In addition to the $\mathrm{G} t_{0}$-refit also the RPC-time correction method was used for the MDT chambers in the middle barrel station (BM) which are located closely to the RPC trigger chambers. The time measured by these RPC can be used to correct for a global time offset. An example of the effectiveness of the method is given in Fig. 7 where the drift time distribution for a BML chamber is shown after RPC-time 
corrections. The steepness of the rising edge, measured as one of the parameters of the Fermi distribution, is improved, passing from $22 \mathrm{~ns}$ without correction, to $3 \mathrm{~ns}$ with RPC time corrections, a value in agreement with results from muon beam tests [5]. The precision of the RPC-time correction is about $2 \mathrm{~ns}$ as explained in Sect. 6.2. This also includes the contribution of the signal propagation time in the RPC strips.

The distribution of the difference between the fitted $\mathrm{G} t_{0}$ and the RPC timing correction per segment is shown in Fig. 11 for a BML chamber. The standard deviation of about $4 \mathrm{~ns}$ is consistent with an uncertainty of $2 \mathrm{~ns}$ from the RPCtime correction added to an uncertainty of $3 \mathrm{~ns}$ introduced by the $\mathrm{G} t_{0}$-refit method. Tails up to $30 \mathrm{~ns}$ are present in the distribution due to bad hit topologies and background hits.

\subsubsection{Drift tube spatial resolution}

The MDT single tube resolution, as a function of drift distance, was studied using different time corrections. The extraction of the resolution function is based on an iterative method. At the first iteration an approximate input resolution function is assumed. Only segments with a minimum of six hits are considered. These segments are fitted again after removing one hit at the time. Subsequently, the width of the distribution of the residuals for the excluded hit, $j$, is computed as a function of the drift distance from the wire, $\sigma_{\mathrm{fit}, j}(r)$. The errors of the straight line fit (depending on the assumed tube resolution) are then propagated to the excluded hit. The resolution $\sigma_{j}(r)$ is then computed by quadratically subtracting from the standard deviation of the residuals the fit extrapolation error, $\sigma_{\mathrm{extr}, j}(r)$ :

$\sigma_{j}(r)=\sqrt{\sigma_{\mathrm{fit}, j}^{2}(r)-\sigma_{\mathrm{extr}, j}^{2}(r)}$

The procedure is iterated using the new resolution function until the input and output resolutions agree within statistical uncertainties; a small number of iterations (two to four) is usually needed.

In Fig. 12 the tube resolution obtained for a BML chamber is shown as the green band. The width of the band accounts for the systematic uncertainty of the method. Also shown (solid line) is the resolution function obtained for an MDT chamber at a high energy muon test beam [5] with well controlled trigger timing. This can be considered as reference for the single-tube resolution. The resolution function measured with cosmic rays is consistent with a time degradation of the reference resolution of about $3 \mathrm{~ns}$. This is in reasonable agreement with the $2 \mathrm{~ns}$ time resolution quoted for the RPC-time correction in addition to a small contribution from multiple scattering and individual tube differences in $t_{0}$.

The single hit spatial resolution was determined also by applying the $\mathrm{G} t_{0}$-refit method to track segments reconstructed in the same chamber. The procedure was similar

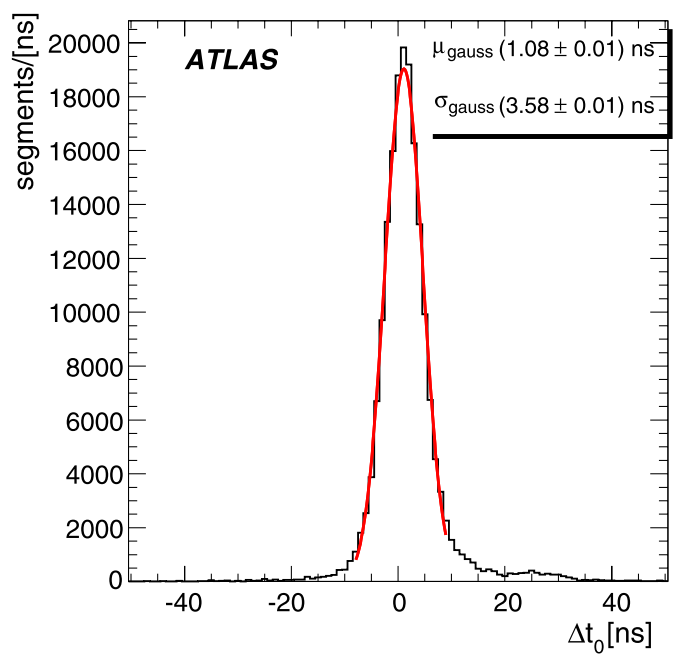

Fig. 11 Difference between the $\mathrm{g} t_{0}$ obtained with the $\mathrm{G} t_{0}$-refit method and with RPC-time correction. The width of the distribution is a convolution of the uncertainties of the RPC-time correction and the $\mathrm{G} t_{0}$-refit method

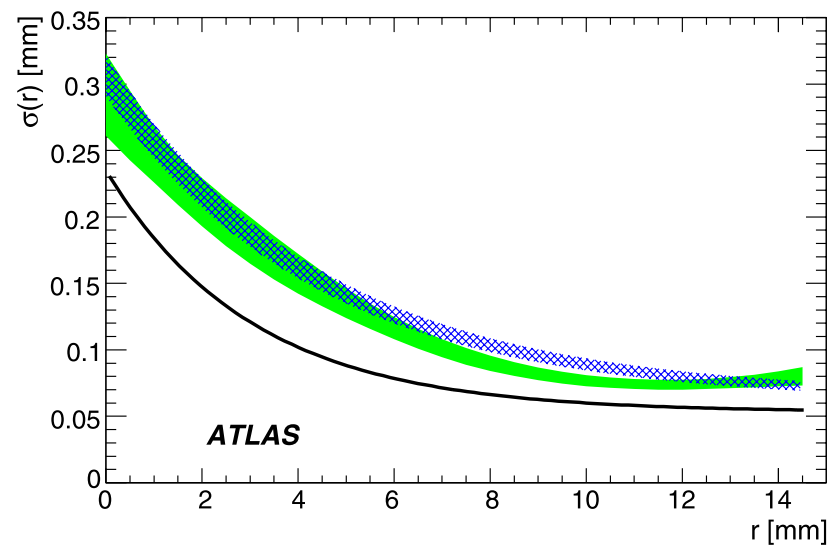

Fig. 12 Drift tube resolution as a function of the radius. The green shadowed (RPC correction method) and the blue hatched ( $\mathrm{G} t_{0}$-refit method) bands represent the resolution function measured with cosmic rays with the two different methods described in the text. The solid line represents the resolution measured with a high momentum muon beam [5]

to that presented above with the convergence of the method driven by the estimate of the residual pulls. The resolution function is shown as the blue hatched band in Fig. 12. The measured resolution is consistent with the test beam measured resolution provided that an additional time uncertainty of about $2-3$ ns is taken into account.

\subsubsection{Drift tube noise}

The level of noise can be measured in each drift tube by looking at the drift time distribution in a given interval before $t_{0}$ where only hits uncorrelated with the trigger are present. The noise rate is obtained by dividing the number of 
hits normalized to the number of triggers by the chosen time interval. The charge of drift tube signals, at nominal running conditions, is well above the ADC pedestal corresponding to about 50 counts, see Fig. 7. In the reconstruction algorithms only hits with charge above this value are considered. The distribution of noise rate with and without the ADC charge cut is shown in Fig. 13 for all MDT drift tubes. The average noise rate is only $60 \mathrm{~Hz}$ without the ADC cut and $13 \mathrm{~Hz}$ with ADC cut, the former figure corresponds to an average tube occupancy of less than $10^{-4}$.

\subsubsection{Drift tube efficiency}

The single tube efficiency was studied by reconstructing segments in a chamber using all tubes except the one under observation i.e. excluding one MDT layer at the time in segment reconstruction. Two different types of inefficiencies can be defined: (i) absence of a hit in the tube; (ii) a hit is present but is not associated to the segment because its residual is larger than the association cut. The inefficiency of type (i), referred to as hardware inefficiency, is very small, mostly occurring at large drift distances, near the tube wall, where the short track length results in fewer primary electrons or due to the track passing through the dead material between adjacent tubes. The inefficiency of type (ii), referred to as tracking inefficiency, is dominated by $\delta$-electrons, produced by the muon itself, which can mask the muon hit if the $\delta$-electron has a smaller drift time than the muon. Tube noise can be an additional source of this type of inefficiency.

Figure 14 shows the distribution of the signed residuals for hits in the tube of one barrel chamber as a function of the distance of the segment from the wire. A large population at small values of the residual, compatible with the spatial resolution, is visible. Large positive residuals are associated with early hits mainly due to $\delta$-electrons. If a hit is not found

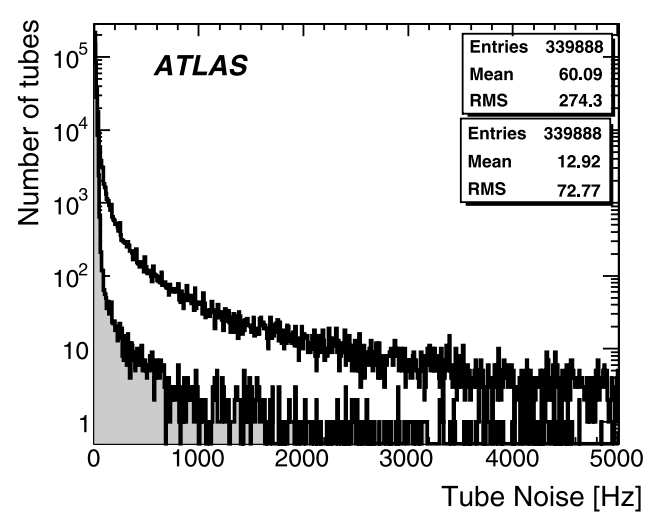

Fig. 13 Distribution of the drift tube noise rate with (shadowed histogram, bottom statistical box) and without (empty histogram, top statistical box) the ADC cut described in the text. In the right plot the logarithmic scale allows observation of the very few noisy tubes.

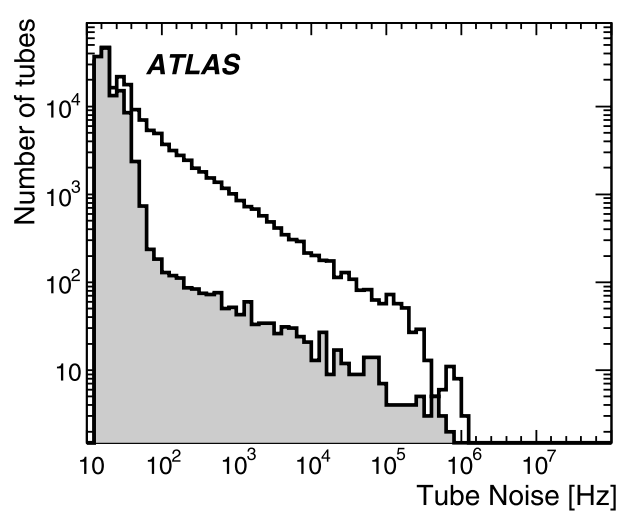

The tail of the distribution is due to very few noisy tubes that are suffering from pick up of high frequencies through the HV cables or interferences due to the digital clock present in the front end electronics
Fig. 14 Distribution of the hit residuals for tubes excluded in the segment fit, as a function of the distance of the track from the wire. Small residuals are associated with efficient hits. The triangular region is populated by early hits produced by $\delta$-electrons. Missing hits, as explained in the text, are assigned a residual value of $15.5 \mathrm{~mm}$. The histogram on the right represent the projection on the residual axis of the plot on the left pane
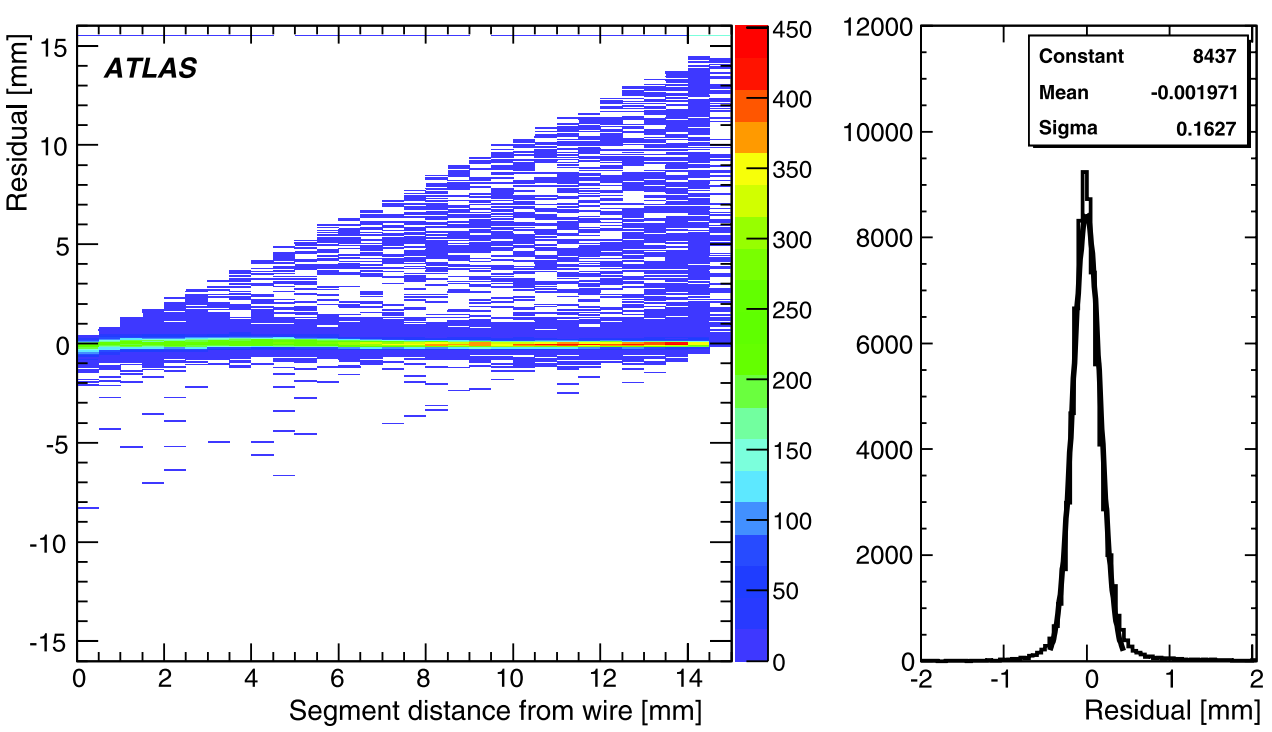
in the tube traversed by the muon (thus a residual cannot be computed) a value of $15.5 \mathrm{~mm}$ is assigned, larger than the tube radius of $15 \mathrm{~mm}$. The population of missing hits is visible at the top of Fig. 14 and it peaks close to the tube wall.

The tracking efficiency is defined as the fraction of hits with a distance from the segment smaller than $n$ times its error, this error being a convolution of the tube resolution and the track extrapolation uncertainty. Figure 15 shows the hardware efficiency and the tracking efficiency as a function of the drift radius for $n=3,5$, and 10. The tracking efficiency decreases with increasing radius, mainly due to the contribution of $\delta$-electrons. The average tube hardware efficiency is $99.8 \%$; the tracking efficiency is $97.2 \%, 96.3 \%$ and $94.6 \%$ for $n$ equals to 10,5 and 3 respectively.

Figure 16 shows the average value of the tracking efficiency for each tube of a BML chamber for $n=5$. The average value is about $96 \%$. An efficiency consistent with zero was obtained for two tubes as can be seen in the expanded view on the right plot. These were recognized as tubes with

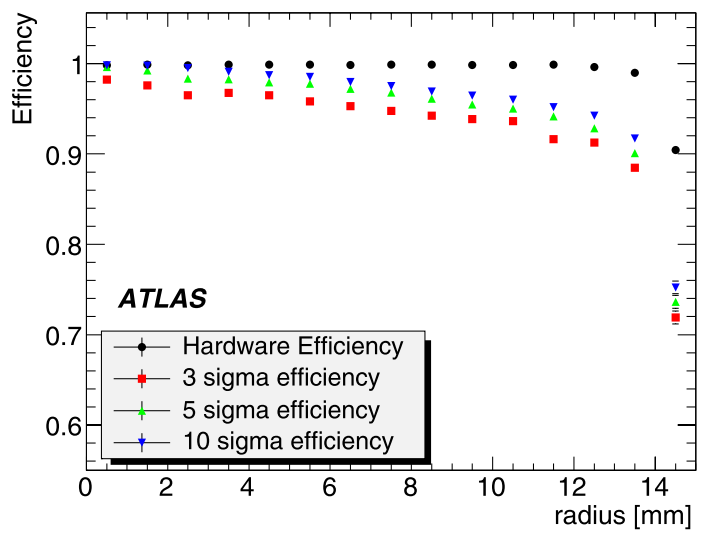

Fig. 15 Tube efficiency as a function of the drift distance averaged over all tubes of a BML chamber. Shown are the hardware efficiency and the tracking efficiency for hit residuals smaller than 3, 5, and 10 times the standard deviation of the distribution disconnected wires and were not considered in the average value.

The results of a study on all the barrel chambers with enough cosmic ray illumination to allow the determination of the single tube efficiency is presented in Fig. 17. The distribution of the tracking efficiency for a $5 \sigma$ hit association cut is shown for about $81 \mathrm{~K}$ drift tubes. In addition to about $0.2 \%$ of dead channels, less than $1 \%$ of tubes have tracking efficiency below $90 \%$, mainly due to calibration constants determined with insufficient precision.

\subsection{RPC}

In addition to providing the barrel muon trigger, the RPC system is also used to identify the BC of the interaction that produced the muon. This requires a time resolution much better than the bunch crossing period of $25 \mathrm{~ns}$. For this, the time of the strips that form the trigger coincidence is encoded in the front-end with a 3-bit interpolator providing an accuracy of $3.125 \mathrm{~ns}$ [10]. The distribution of the time difference between the two layers of a pivot plane in the $\phi$ projection was used to determine the RPC time resolution. With this method there is no need to correct for the muon time of flight and the signal propagation along the read-out strips. The RMS width of the distribution shown in Fig. 18 is $2.5 \mathrm{~ns}$. From this a time resolution of $1.8 \mathrm{~ns}$ is derived for the two RPC layers forming the coincidence. For this measurement only strips associated to a reconstructed muon track and belonging to events with one and only one RPC trigger were considered.

Two other important RPC quantities related to the detector performance are the efficiency and the spatial resolution. In order to determine the RPC efficiency two main issues have to be taken into account. The first one is due to the fact that the RPCs are actually providing the muon trigger thus resulting in a trigger bias on the efficiency calculation. The second one is caused by the fact that the RPC hits are
Fig. 16 Single tube tracking efficiencies with a $5 \sigma$ association cut, as explained in the text, for a BML chamber. The plot on the right shows an expanded view in the region where two tubes with the wire disconnected were found
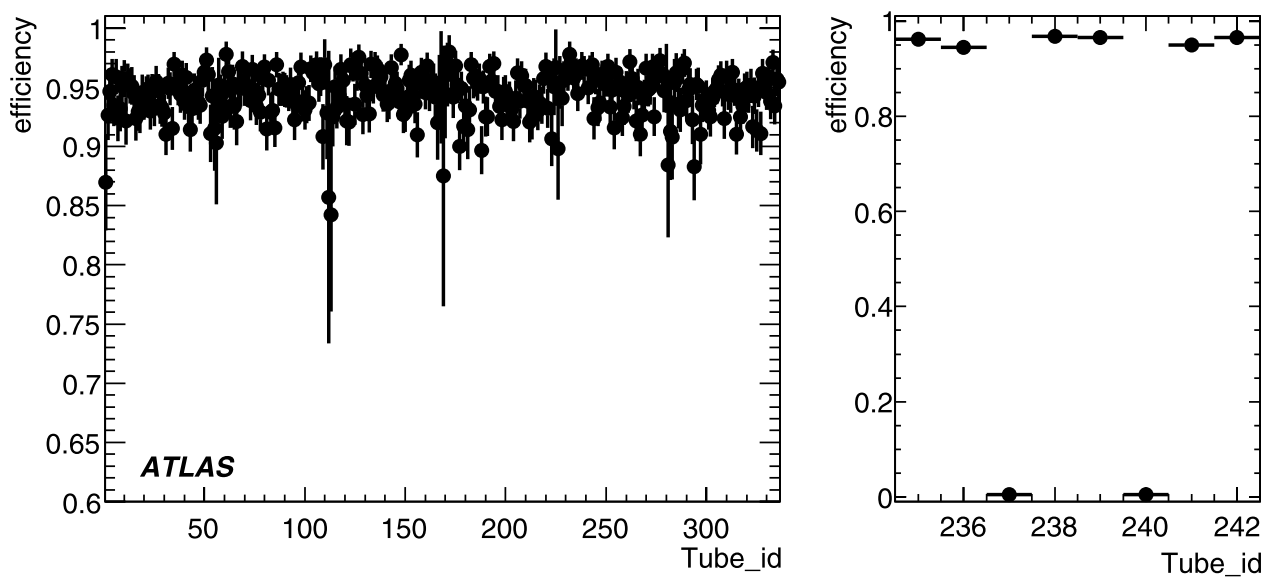


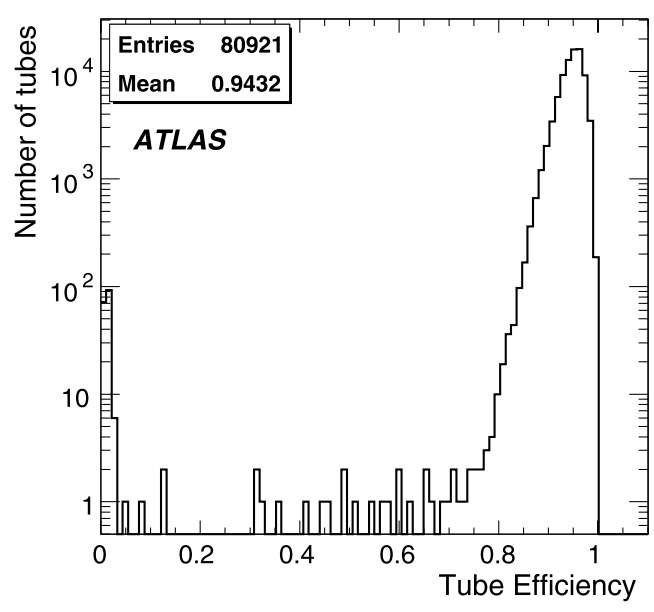

Fig. 17 Distribution of the tracking efficiency, with a $5 \sigma$ hit association cut, for $\sim 81 \mathrm{~K}$ drift tubes in the barrel MDT. About $0.2 \%$ of tubes were not working and have efficiency compatible with zero

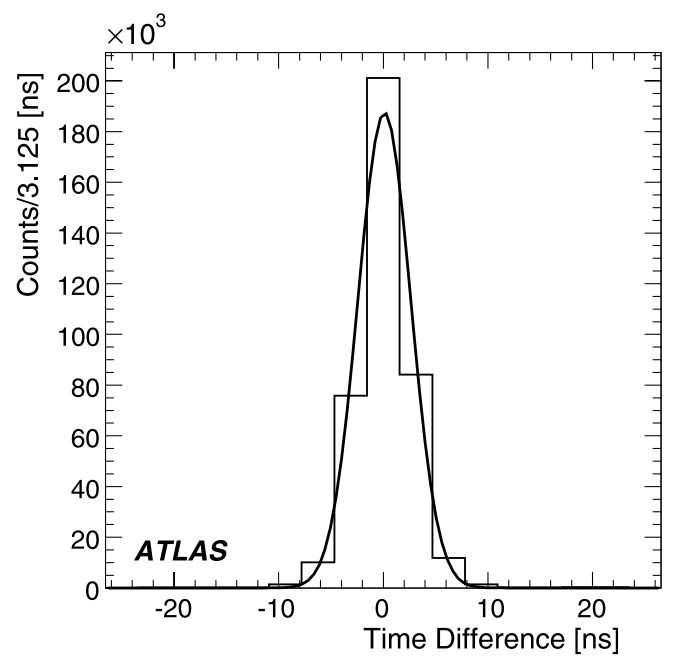

Fig. 18 Distribution of the time difference between the two RPC layers of a pivot plane in the $\phi$ projection. The binning of the histogram corresponds to the strip read-out time encoding of $1 / 8$ of BC

also used in the track reconstruction; in particular, they measure the coordinate in the non-bending, $\phi$ projection. The second effect has negligible contribution if the efficiency is measured for the $\eta$ strips, since in this projection the track reconstruction is driven by the MDT. For the efficiency measurement, MDT tracks were extrapolated to the RPC plane and the layer was counted as efficient if at least one $\eta$ hit was found with a distance of less than $7 \mathrm{~cm}$ from the extrapolation. The effect of the trigger bias has been removed from the efficiency measurement of an RPC plane by selecting all the events in which the other three planes (in the case of a Middle Station) were producing hits, since the trigger requirement is a 3 over 4 planes majority. The distribution of the efficiency, averaged over each layer, for the RPC chambers in the Middle stations is shown in Fig. 19, the dis-

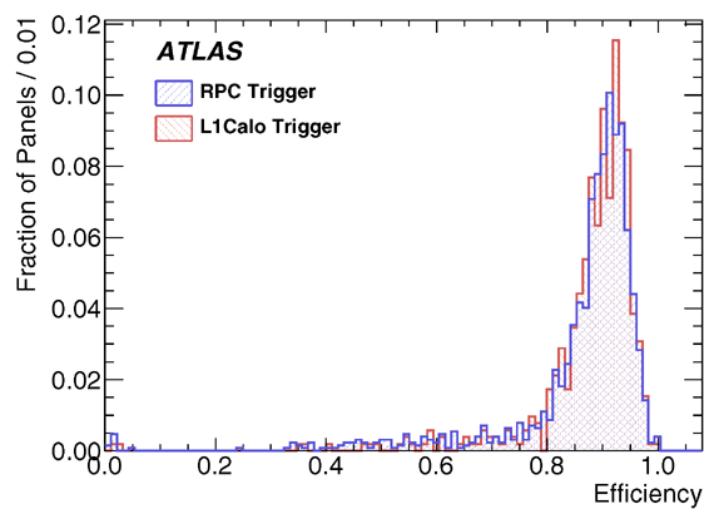

Fig. 19 Distribution of the average efficiency for RPC of the Middle stations for run 91,060 . The two distributions refer to two different triggers: RPC trigger (full line, $91.33 \%$ peak efficiency) and calorimeter trigger (dashed line, $92.0 \%$ peak efficiency). Both distributions are normalized to unit area. The measured efficiency is lower than expected mainly because the read-out timing was still not optimal

tribution is peaked at an efficiency of $91.3 \%$. To check the remaining impact of the trigger bias on the efficiency measurement, the same analysis was repeated with a sample of cosmic rays selected with a calorimeter trigger (Level-1Calo trigger) independently of the RPC trigger response. The result for the efficiency is superimposed in Fig. 19: a good agreement between the two distributions is observed.

The spatial resolution is related to the clusters size, that is the number of strips associated to a muon track. A muon crossing the detector near the center of a readout strip, will in general produce a cluster of size one, while clusters of size two are only observed when muons hit a narrow region at the boundary between two strips. The actual sizes of the regions corresponding to clusters of size one and two depends on the detector operating parameters, but it is in general true that the latter is smaller than the former. This implies that the spatial resolution must be smaller when measured on a subset of data with only clusters of size two. The spatial resolutions of $\eta$ strips was determined selecting muon tracks reconstructed in the MDT as explained above. For each RPC read out plane, the distribution of the distance from the extrapolated track was obtained separately for clusters of size one and two and then was fitted with a Gaussian. The RMS widths of the fit were divided by the strip pitch (ranging from 27 to $32 \mathrm{~mm}$ depending on the chamber type) to allow for comparison between different RPC and are shown in Fig. 20. This technique has been used only for the $\eta$ panels since the MDT are measuring in the $Z-Y$ plane. On average, clusters of size two give a spatial resolution about half as for clusters of size one, which is below $10 \mathrm{~mm}$ as expected.

\subsection{TGC}

The basic structure of the TGC chambers and their assembly in the MS end-cap wheels is presented elsewhere [2]. Inactive regions due to the gas-gap frame and the wire supports 
account for a loss of active area varying from $3 \%$ to $6 \%$ depending on the chamber type. In order to optimize the trigger efficiency these inactive regions are staggered with respect to the trajectory of high momentum muons produced at the IP. In the active area the TGC wires are expected to have an efficiency of more than $98 \%$. For the cosmic ray run 91,060 the trigger logic required a coincidence of 3 out of 4 layers in the doublet chambers (referred to as TGC2 and TGC 3 as in Fig. 1). In evaluating the detector efficiency one has to take into account the trigger bias and the fact that cosmic rays are non-pointing to the IP, asynchronous, and do not only consist of single muons but also of extended showers.

To evaluate the efficiency of a layer in the doublet chambers, it is required that there is one and only one hit in each of the other three layers and that these three hits are associated to the current BC. This is intended to remove high multiplicity events (showers) and out-of-time tracks. As a result of this selection, the 3 out of 4 trigger condition is satisfied independently of the presence of a hit in the layer under evaluation. The efficiency of this layer is thus determined in an unbiased way.

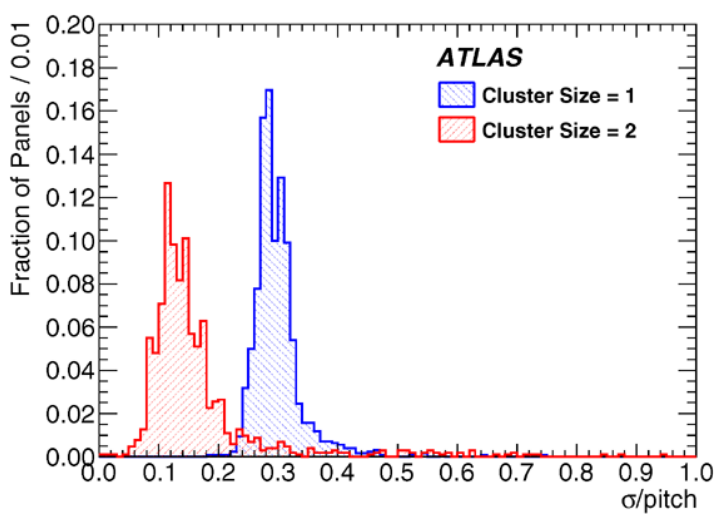

Fig. 20 Distribution of the spatial resolution provided by the $\eta$ strips for RPC of the Middle stations. The spatial resolution is divided by the strip pitch. The distributions are normalized to unit area
A similar procedure is used for the triplet chambers (TGC1). When evaluating, the efficiency of a layer, it is required (i) that the other two layers satisfy the 2 out of 3 trigger coincidence and (ii) that the line joining the two hits (track) crosses the layer in its active area.

In both cases, the layer under test is considered efficient if there is at least one hit associated to any of the previous, current or next BC. Figure 21 on the left shows an efficiency map in the wire-strip $(\eta-\phi)$ plane, and on the right its $\eta$ projection, i.e. the strip efficiency. Some inactive regions are clearly visible: the bands in Fig. 21—Left indicate the location of the wire supports.

The overall efficiency, including the inactive regions, is evaluated for a fraction of TGC layers (about $40 \%$ of TGC doublet layers) by requiring that a muon track crosses the layer under test at least $10 \mathrm{~cm}$ away from its edge. The muon track is defined using MDT hits combined with TGC hits in the layers that are not under evaluation. Figure 22 shows the distribution of the wire efficiency for different values of high voltage setting: 2650, 2750, 2800 and $2850 \mathrm{~V}$. The average value of the efficiency, at the nominal voltage of $2800 \mathrm{~V}$, is $92 \%$ consistent with the local efficiency measured as explained above and the contribution from inactive-regions.

\section{MDT optical alignment}

The design transverse momentum resolution at $1 \mathrm{TeV}$ of the MS is about $10 \%$, this translates into a sagitta resolution of $50 \mu \mathrm{m}$. The intrinsic resolution of MDT chambers contributes a $40 \mu \mathrm{m}$ uncertainty to the track sagitta, hence other systematic uncertainties (alignment and calibration) should be kept at the level of $30 \mu \mathrm{m}$ or smaller. Since long-term mechanical stability in a large structure such as the MS cannot be guaranteed at this level, a continuously running alignment monitoring system [21] has been installed. This system is based on optical and temperature sensors and detects slow chamber displacements, occurring at a timescale of hours or
Fig. 21 Left: efficiency map for a TGC chamber layer. The horizontal axis is the strip channel and the vertical axis is the wire channel. Right: efficiency projection to the strip channels. Observed efficiency drops are consistent with the wire support locations
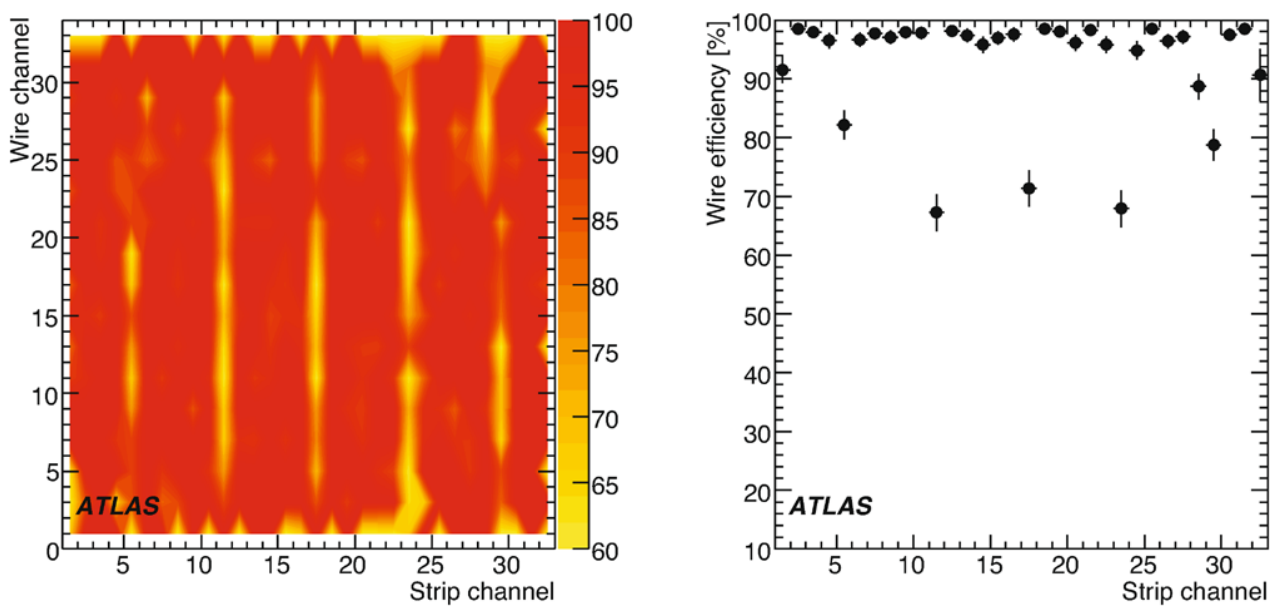

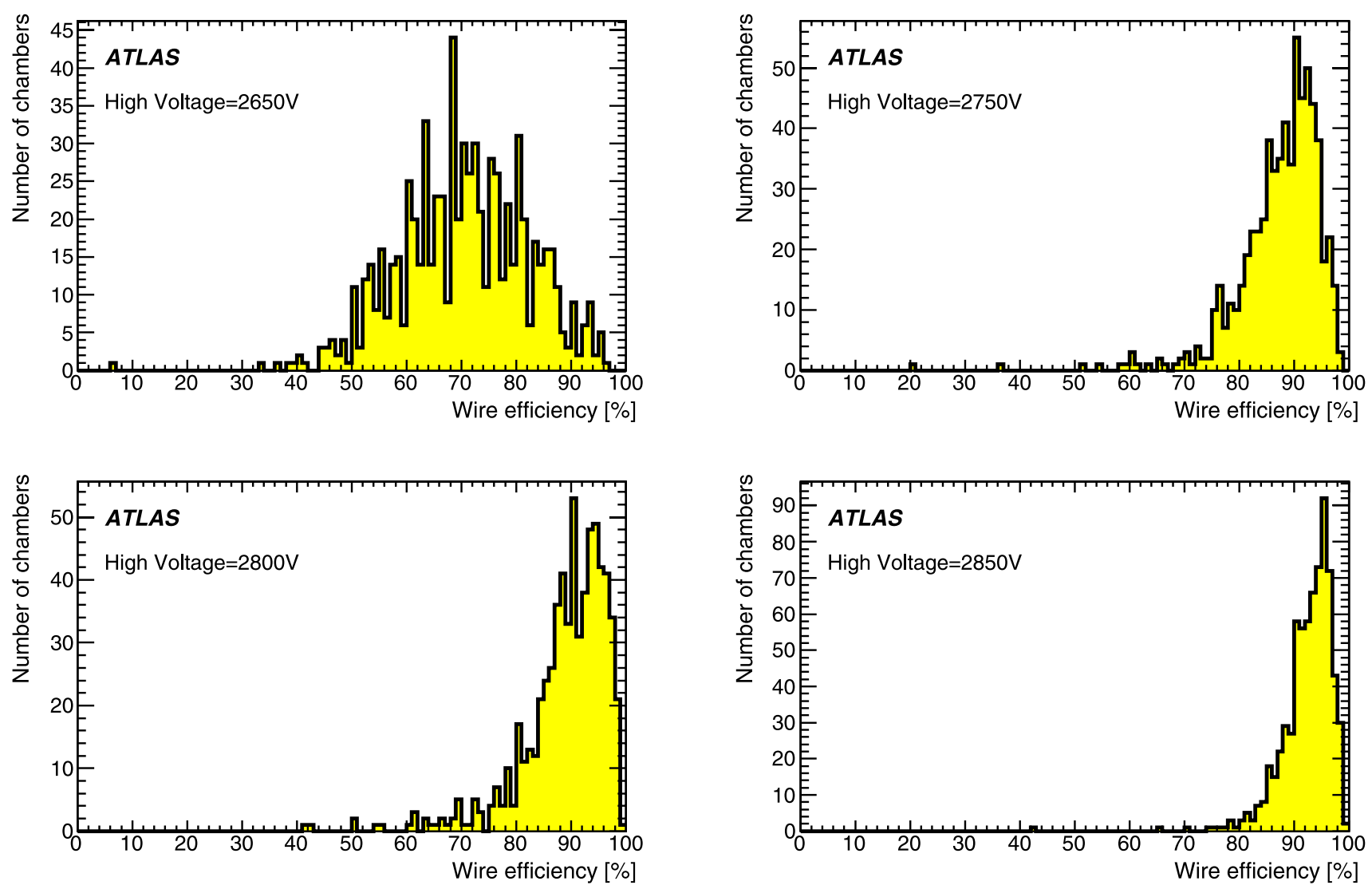

Fig. 22 Distribution of the TGC wire efficiency of individual layers for different high voltage values. The distribution for $2800 \mathrm{~V}$, the nominal voltage in 2008, was obtained with run 91,060

more. The information from the alignment system is used in the offline track reconstruction to correct for the chamber misalignment. No mechanical adjustments were made to the chambers after the initial positioning. The system consists of a variety of optical sensors, all sharing the same design principle: a source of light is imaged through a lens onto an electronic image sensor acting as a screen. In addition to optical position measurements, it is also necessary to determine the thermal expansion of the chambers. In total, there are about 12,000 optical sensors and a similar number of temperature sensors in the system. Optical and temperature sensors were calibrated before the installation such that they can be used to make an absolute measurement of the chamber positions in space, rather than only following their movements with time relative to some initial positions.

\subsection{End-cap chamber alignment}

The end-cap chambers and their alignment system [22] were installed and commissioned during 2005-2008, and continuous alignment data-taking with the complete system started in Summer 2008. After commissioning, more than $99 \%$ of all alignment sensors were operational, and only a small number failed during the data-taking in 2008. The effect of the missing sensors on the final alignment quality is negligible.

The position coordinates, rotation angles, and deformation parameters of the chambers are determined by a global $\chi^{2}$ minimization procedure. The total $\chi^{2}$, as well as the contributions of the individual sensor measurements to the $\chi^{2}$ (pulls) can be used to estimate the alignment quality from the internal consistency of the fit. If the observed sensor resolutions agree with the design values, one expects approximately $\chi^{2} / \mathrm{ndf}=1$ and a pull distribution with zero mean and unit RMS width. Figure 23 shows the observed and expected pull distributions in the end-caps, obtained by assuming the design resolutions for all sensor types.

In a second step, the assumed sensor resolutions are adjusted until the observed pull distributions, broken down by sensor type, agree with the expected distribution. This yields the observed sensor resolutions, which are used as input to a Monte Carlo simulation of the alignment system. The simulation predicts a sagitta accuracy due to alignment of about $45 \mu \mathrm{m}$, which is close to the design performance.

Validating the alignment as reconstructed from the optical sensor measurements requires an external reference. During chamber installation, surveys of the completed endcap wheels were done using photogrammetry, and the cham- 


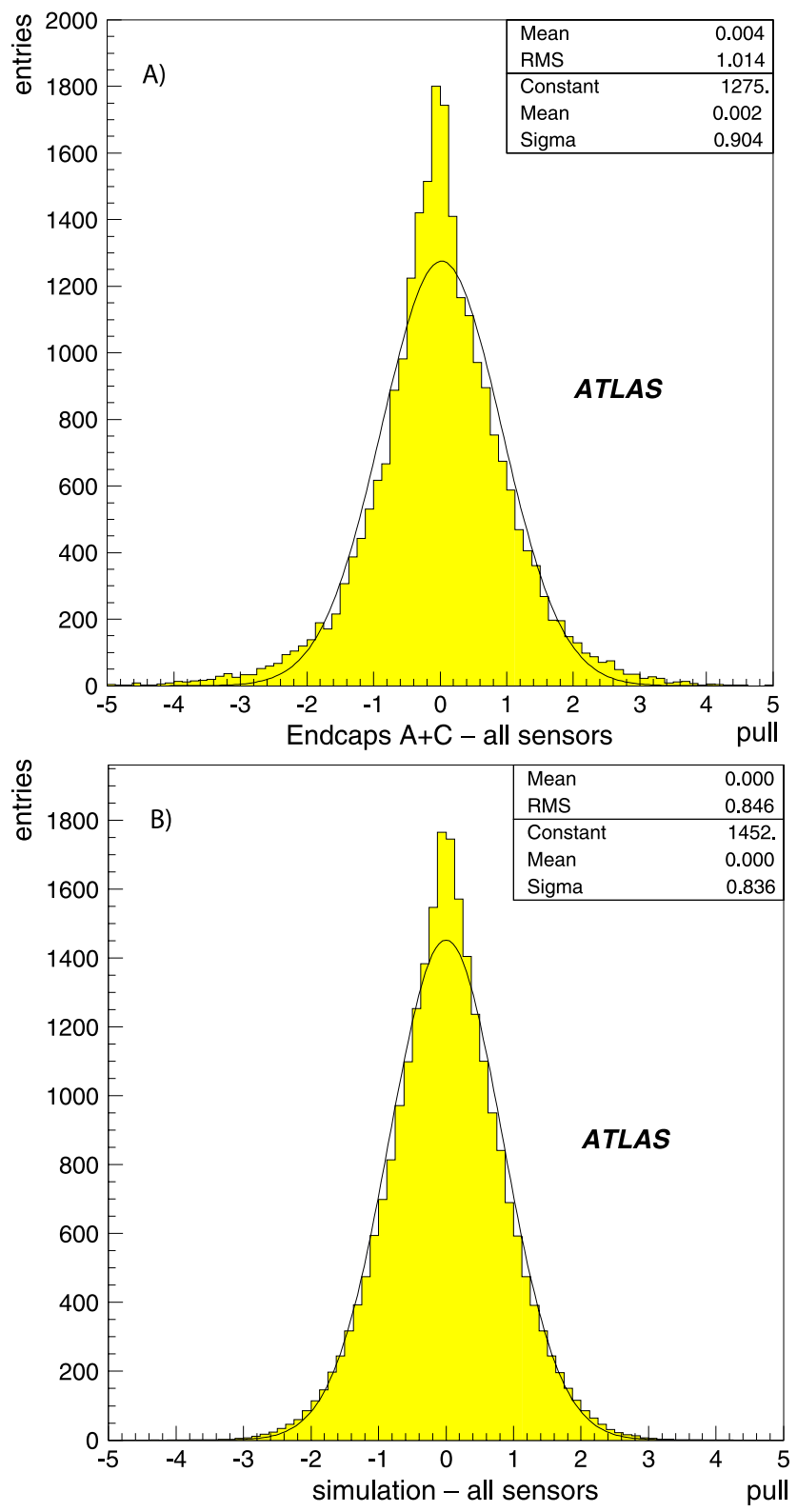

Fig. 23 The observed (A, from data) and expected (B, from simulation) pull distributions for the end-caps, assuming design resolution for all sensor types. Correlations and weakly constrained degrees of freedom cause the expected pull distribution to have RMS width below unity. The observed $\chi^{2} /$ ndf from the fit on data is 1.4 , while the one from simulation is 1.0

ber positions measured with the alignment system agreed with the survey results within $0.5 \mathrm{~mm}$, the quoted accuracy of the survey. While establishing confidence in the optical system, the full validation of the alignment can only be done with tracks. Thus, cosmic muons recorded during magnetoff running were used to cross-check the alignment provided by the optical system.

For a perfect alignment, the reconstructed sagitta of straight tracks should be zero for each EI-EM-EO measurement tower (note that, when averaged over many towers, the mean value can be accidentally compatible with zero despite single towers being significantly misaligned). For cosmic muons, the observed width of the sagitta distribution is dominated by multiple scattering. A shifted and/or broadened distribution would indicate imperfections of the alignment. Triplets of track segments were selected in the EIEM-EO chambers, requiring the three segments to be in the same sector and assigned to the same reconstructed track. Some segment quality cuts were applied for this analysis: (i) $\chi^{2} /$ ndf $<10$ and at most one expected hit missing per chamber; (ii) the angle between the segments and the straight line joining the segments in EI and EO was required to be smaller than 5 (50) mrad in the precision (second) coordinate; (iii) at least one trigger hit in the second coordinate was required to be associated to the track. A total of 1700 segment triplets passing the cuts were selected in run 91,060 .

Figure 24(A) shows, for the two end-caps, the observed sagitta distribution before and after applying alignment corrections (i.e. the chamber positions, rotations, and deformations as determined by the optical system, as well as a correction for the gravitational sag of the MDT wires). Figure 24(B) shows the corresponding difference in angle in the precision coordinate between each of the segments and the track (the straight line joining the EI and EO segments). For the distribution in Fig. 24(B), the cut at $5 \mathrm{mrad}$ was omitted. The improvement in both variables is clearly visible, the mean value of the corrected sagitta distribution as obtained from the fit with a double-Gaussian function is $(-33 \pm 42) \mu \mathrm{m}$ and thus perfectly compatible with zero within the $45 \mu \mathrm{m}$ error estimated above from the internal consistency of the alignment fit. The width of the corrected sagitta distribution agrees approximately with expectations for the typical energies of triggered cosmic muons. The width of the corrected angle distribution, on the other hand, is about twice as large as expected. This is mainly a consequence of the additional time jitter of MDT measurements described in Sect. 5 which deteriorates the segment resolution.

For the two end-caps separately, the mean value of the sagitta distribution is $(-30 \pm 61) \mu \mathrm{m}$ in side $\mathrm{A}$ and $(-37 \pm$ 57) $\mu \mathrm{m}$ in side $C$. The sign of the sagitta is defined in such a way that most of the conceivable systematic errors would cause deviations from zero with the same sign in side A and side $\mathrm{C}$. The analysis is limited by statistics even though it uses a significant fraction of the full 2008 data sample. Breaking it down further to the level of sectors, or even to projective towers (where the best sensitivity is obtained) would require significantly more data.

The cross-check with straight tracks confirms that, with the limitations of the analysis, the chamber positions given by the optical alignment system are within the estimated sagitta uncertainties, indicating that the optical system 


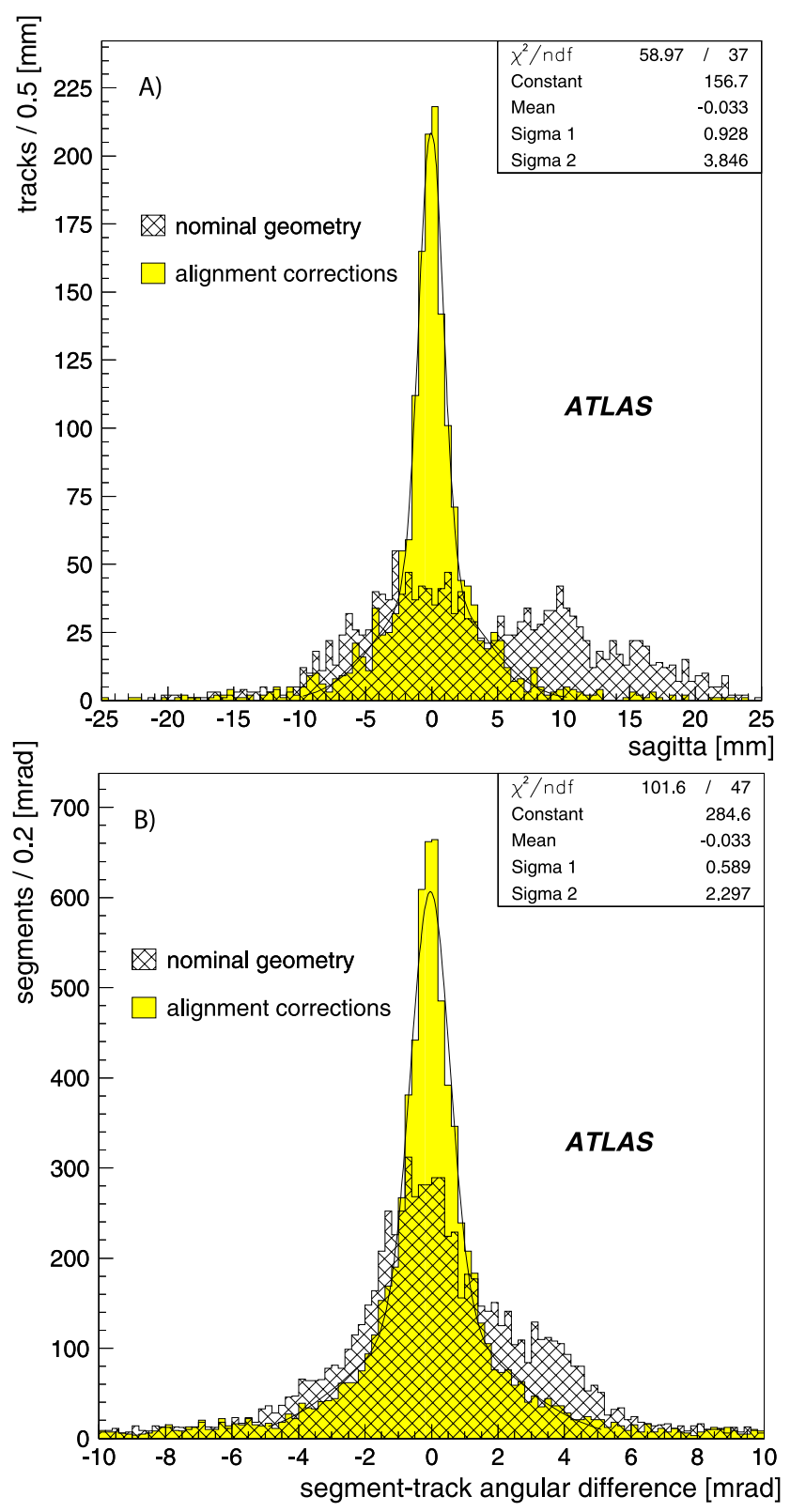

Fig. 24 (A): Measured sagitta distribution for the two end-caps. The cross-hatched histogram shows the sagitta before alignment corrections, thus reflecting the accuracy of chamber positioning. The filled histogram shows the sagitta after applying alignment corrections, the curve is the fit of a double-Gaussian function, each Gaussian containing $50 \%$ of the events. (B): Measured angle in the precision coordinate between the segments and the track to which they are associated

works properly. The design accuracy has nearly been reached in the end-caps. It also shows that the system produces a reliable estimate of the uncertainty of the alignment corrections.

\subsection{Barrel chamber alignment}

The installation and commissioning of the barrel optical system [23] began in 2005 and continued together with the installation of the chambers until 2008. At the time of record-
Table 4 Status of the barrel optical system in Fall 2008. No data were recorded during this period from the "broken" sensors. Naming and functions of the different sensors are detailed in reference [23]

\begin{tabular}{lrcc}
\hline Type & Total & Working & Broken \\
\hline Projective & 117 & 117 & 0 \\
Axial & 1036 & 1031 & 5 \\
Praxial & 2010 & 2008 & 2 \\
Reference & 256 & 253 & 3 \\
CCC & 260 & 260 & 0 \\
BIR-BIM & 32 & 32 & 0 \\
Inplane & 2110 & 2101 & 9 \\
Total & 5817 & 5798 & 19 \\
$\%$ & & 99.7 & 0.3 \\
\hline
\end{tabular}

ing cosmic ray data, the barrel optical system was fully installed and $99.7 \%$ of the sensors were functioning correctly. Table 4 summarizes the status of the 5817 installed sensors. The complete system is read out continuously, at a rate of one cycle every 20 minutes. The readout was functioning correctly during the complete period of acquisition of cosmic ray data.

The alignment reconstruction consists in determining the chamber positions and orientations (referred to as "alignment corrections") from the optical sensor measurements. This requires the precise knowledge of the positions of the sensors with respect to the MDT wires. To this purpose, the optical sensors were calibrated before installation and their mechanical supports were glued with precise tools onto the MDT tubes. However, the original design of the barrel optical system suffered from a few errors that eventually degraded the precision of the alignment corrections. Furthermore, the only devices giving projective information in the Small sectors are the CCC sensors which are designed to provide $1 \mathrm{~mm}$ accuracy. The alignment of the chambers of the Small sectors is, by design, based on tracks that cross the overlap region between the Small and the Large sectors. However, the statistics obtained in cosmic runs was not sufficient to perform a precise check of this method.

The alignment corrections discussed here cover the nine upper sectors (1 to 9). The complete period of cosmic data taking was divided in intervals of 6 hours, and alignment corrections were reconstructed using the sensor measurements recorded in that interval. This provided data for monitoring of significant movements of the MS, e.g. when the magnetic field in the toroids was switched on.

The barrel alignment reconstruction is based on the minimization of a $\chi^{2}$, whose inputs are, for each optical sensor $i$ :

- the recorded response $\mathbf{r}_{i}$

- a model $\mathbf{m}_{i}(\mathbf{a})$, representing the predicted response of sensor $i$ with respect to the alignment corrections a

- the error $\sigma_{i}$, the estimated uncertainty of the model $\mathbf{m}_{i}$. 
The critical part is the model $\mathbf{m}_{i}$, as it combines all the knowledge of the precise geometry of the optical sensors and their calibration. The free parameters in the fit are the alignment corrections a, and in some cases additional parameters used to model the effect of imprecise sensor positioning or of an incorrect calibration. For all these additional parameters appropriate constraints are included in the fit reflecting the best estimates of the error contributions mentioned above. Overall, 4099 parameters are fit simultaneously. The total reconstruction time for the full barrel is less than one minute.

Given the uncertainties introduced by the additional parameters in the fit procedure, the strategy for alignment in the barrel is slightly different from the one in the end-cap. Dedicated runs without magnetic field in the toroids (but with field in the solenoid to tag high momentum tracks) will be used to get initial alignment corrections with a precision of $30 \mu \mathrm{m}$. The optical alignment system is then used to monitor movements due to the switching on of the toroidal field and to temperature effects. The mechanical stability of the system, in periods where the magnetic field was constant, is at the level of $100 \mu \mathrm{m}$, while movements of the magnet structures at the level of few mm were observed when the magnets were switched on and off. The optical alignment system, which continuously monitors the position of the chambers, is able to follow these movements with the required accuracy. This so-called relative alignment mode has already been tested with success in the MS system-test done with a high-energy muon beam $[4,5]$. After the minimization, the value obtained for $\chi^{2} /$ ndf is 1.9 , which shows that the sensor errors are underestimated.

\subsubsection{Performance of the optical alignment in the barrel}

Similarly to what is done in the end-cap, an estimate of the contribution to the sagitta error due to the alignment system may be inferred from the $\chi^{2}$, using the following formula

$\left(V^{-1}\right)_{i j}=\left.\frac{1}{2} \frac{\partial^{2} \chi^{2}}{\partial \theta_{i} \partial \theta_{j}}\right|_{\hat{\theta}}$

where $\theta_{i}$ are the fitted parameters and $V$ is the global error matrix, of size $4099 \times 4099$, of all fitted parameters. To estimate the performance of the alignment system in terms of sagitta measurement, straight tracks, originating in the IP and crossing three layers of chambers, were simulated and the whole fit procedure was applied to these tracks. The sagitta of these pseudo-tracks is a function of some of the alignment corrections, and thus the formula of error propagation may be used to infer the contribution of the alignment to the error of the resulting sagitta. This technique relies on the hypothesis that the errors of the optical sensors are correctly estimated, and thus that the $\chi^{2}$ is correctly normalized. As this is not the case $\left(\chi^{2} / \mathrm{ndf}=1.9\right)$, the results are

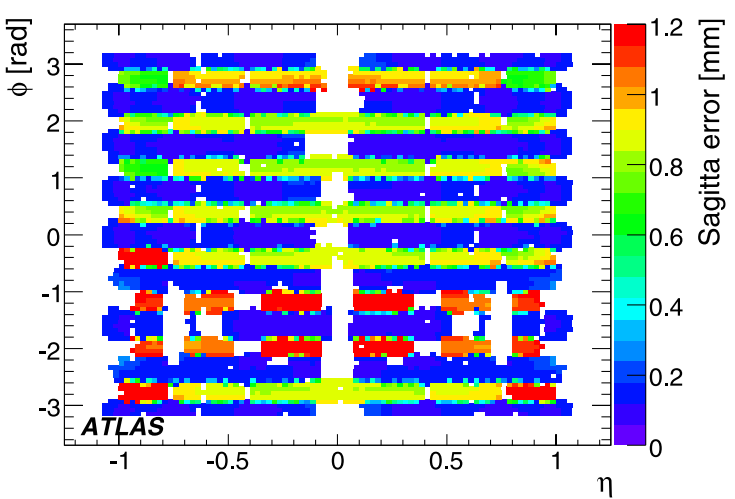

Fig. $25 \eta \times \phi$ map of the contribution to the sagitta error due to alignment, as estimated with the method described in the text. As expected from the system design, the Small sectors (even sector numbers) are aligned with significantly less precision than the Large sectors (odd sector numbers)

only considered as a rough estimate of the optical alignment performance.

The result is shown in Fig. 25. The Small sectors have a significantly worse alignment than the Large sectors, as explained above. Conservatively, one can conclude that the performance of the optical system, in terms of sagitta precision, is $\sim 200 \mu \mathrm{m}$ for the Large sectors, and $\sim 1 \mathrm{~mm}$ for the Small sectors.

\subsubsection{Alignment with straight tracks}

Data with the toroidal field off were used to improve the alignment precision in the barrel and to validate the alignment corrections in relative mode. The method is to use straight muon tracks to determine in absolute mode the initial spectrometer geometry and, once this geometry is determined, to use the optical alignment system to trace all chamber displacements in a relative mode. The alignment procedure with straight tracks is based on the so-called MILLEPEDE fitting method [24]. This method uses both alignment and track parameters inside a global fit. As a result, all correlations between alignment and track parameters are taken into account and the alignment algorithm is unbiased.

The track alignment algorithm has been tested with Monte Carlo simulations and with cosmic ray data. The simulation studies show that $10^{5}$ muon tracks with a momentum greater than $20 \mathrm{GeV}$ and pointing to the IP are needed to align the Large sectors with a precision of $30 \mu \mathrm{m}$. Small sectors require five times more tracks than Large sectors, due to the multiple scattering in the toroid coils.

Using straight cosmic muon tracks recorded in run 91,060 , a set of alignment constants has been produced. A total of $10^{7}$ events were used corresponding to about $3 \times 10^{5}$ cosmic muon tracks in each of the most illuminated barrel sectors. The statistical uncertainty of the sagitta using 
this track alignment procedure was estimated to be $30 \mu \mathrm{m}$ for Large sectors.

The data of run 91,060 were processed with the track reconstruction software twice: (i) using the optical alignment corrections and (ii) using the track-based alignment corrections. Both geometries were then tested by measuring the distribution of the track sagitta for muons crossing three chamber stations (Inner, Middle and Outer). Only tracks passing close to the IP in the $\eta$ projection were chosen. Hits in the Inner and Outer chambers were fit to a straight line, and the distribution of the hit residuals in the Middle chambers was used to evaluate the sagitta. For perfect alignment, the mean value of the sagitta should be zero for straight tracks and, to a good approximation, the mean value of the distribution gives an estimate of the sagitta error.

The results are shown in Fig. 26 for the sets of alignment corrections; on the left for a station in a Large sector, on the right for a station in a Small sector. For reference, the distributions using the design geometry are also shown. The tails of the distributions are due to multiple scattering of muons. In the Large sector station, the two distributions are almost identical, but the distribution with optical alignment is centered at $\sim 100 \mu \mathrm{m}$. In the Small sector station, the distribution with the optical alignment is centered around
$1 \mathrm{~mm}$. To compare the results obtained in different stations, Fig. 27 shows the mean values of the sagitta distribution for the Large upper sectors (3, 5 and 7). One Small sector is also presented, sector 4 , since this was illuminated with enough events during the same run to produce a meaningful distribution.

The results show that the optical alignment system alone provides a precision at the level of $200 \mu \mathrm{m}$. When calibrated with sufficient statistics of high momentum straight tracks, the optical system is able reach a precision of $50 \mu \mathrm{m}$.

The sagitta resolution for runs with no magnetic field in the MS can be studied as a function of the muon momentum measured by the ID. The sagitta resolution as a function of the muon momentum was parameterized as

$\sigma_{s}(p)=\frac{K_{0}}{p} \oplus K_{1}$

where the first term $K_{0}$ is due to multiple scattering in the material of the MS, and the second term $K_{1}$ is due to the single tube resolution and chamber-to-chamber alignment. These two terms have been already measured at the MS system test beam $[4,5]$ and found to be $K_{0}=9 \mathrm{~mm} \times \mathrm{GeV}$ and $K_{1}=50 \mu \mathrm{m}$. A similar measurement was done with cosmic muons by selecting segment triplets (Inner, Middle
Fig. 26 Distribution of the sagitta (as defined in the text) for straight tracks. (A), (B): Using alignment corrections derived from the optical system only; (C), (D): Using track-based alignment corrections. (A), (C): For a station in a Large barrel sector; (B), (D): For a station in a Small barrel sector, the optical system corrections of the small sectors have, by design, an accuracy at a level of $1 \mathrm{~mm}$. In all panels, the hashed distribution is obtained using the "nominal" geometry. Mean and sigma in the statistical boxes refer to the distributions with alignment corrections, the peak is fitted with a Gaussian in a \pm 1 sigma interval
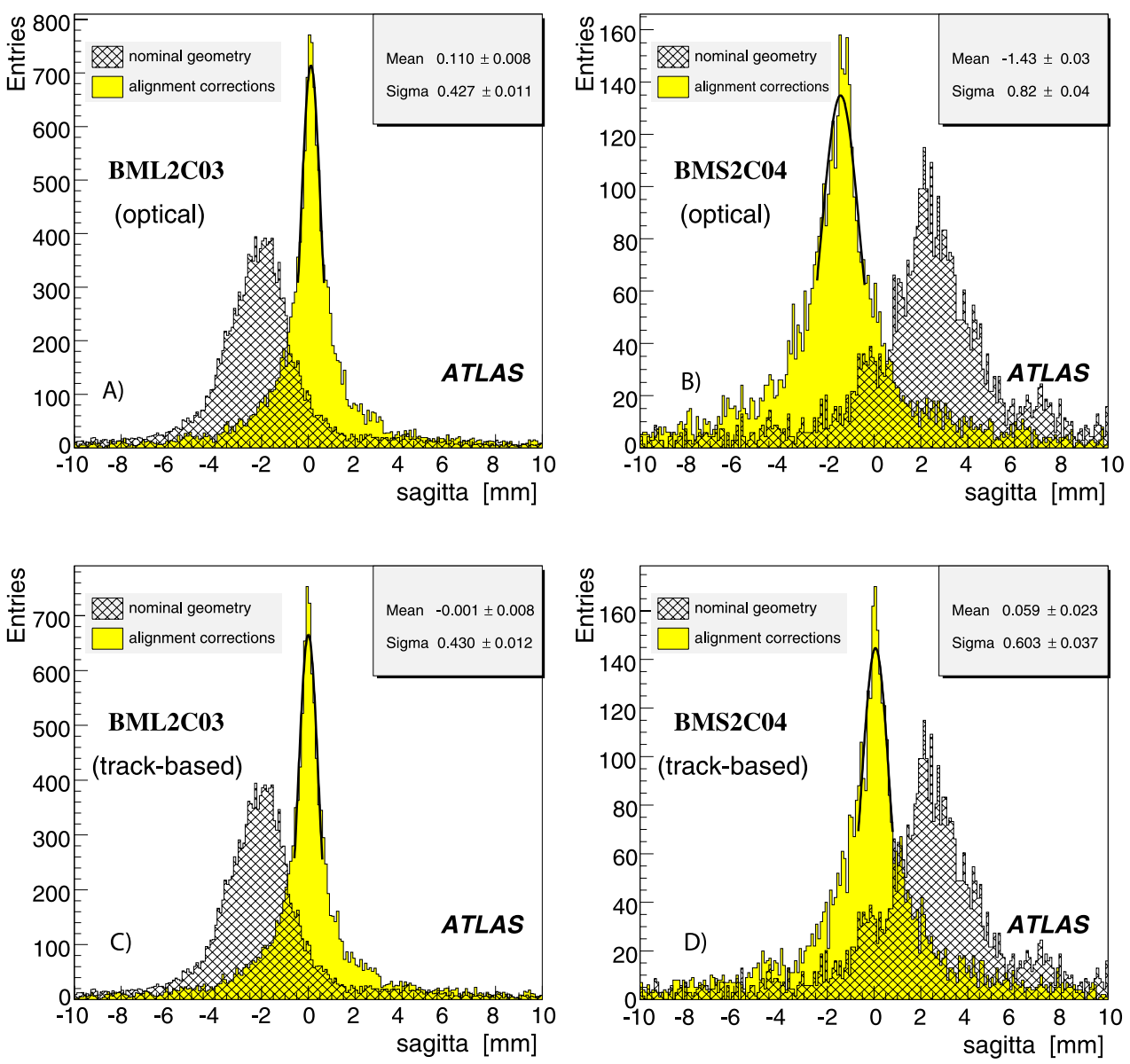
Fig. 27 Mean value of the track sagitta distributions obtained (A), (B): With the optical alignment system only, (C), (D): and using the track-based alignment. (A), (C): For the upper Large barrel sectors. (B), (D): For a Small barrel sector with $56^{\circ}<\phi<79^{\circ}$
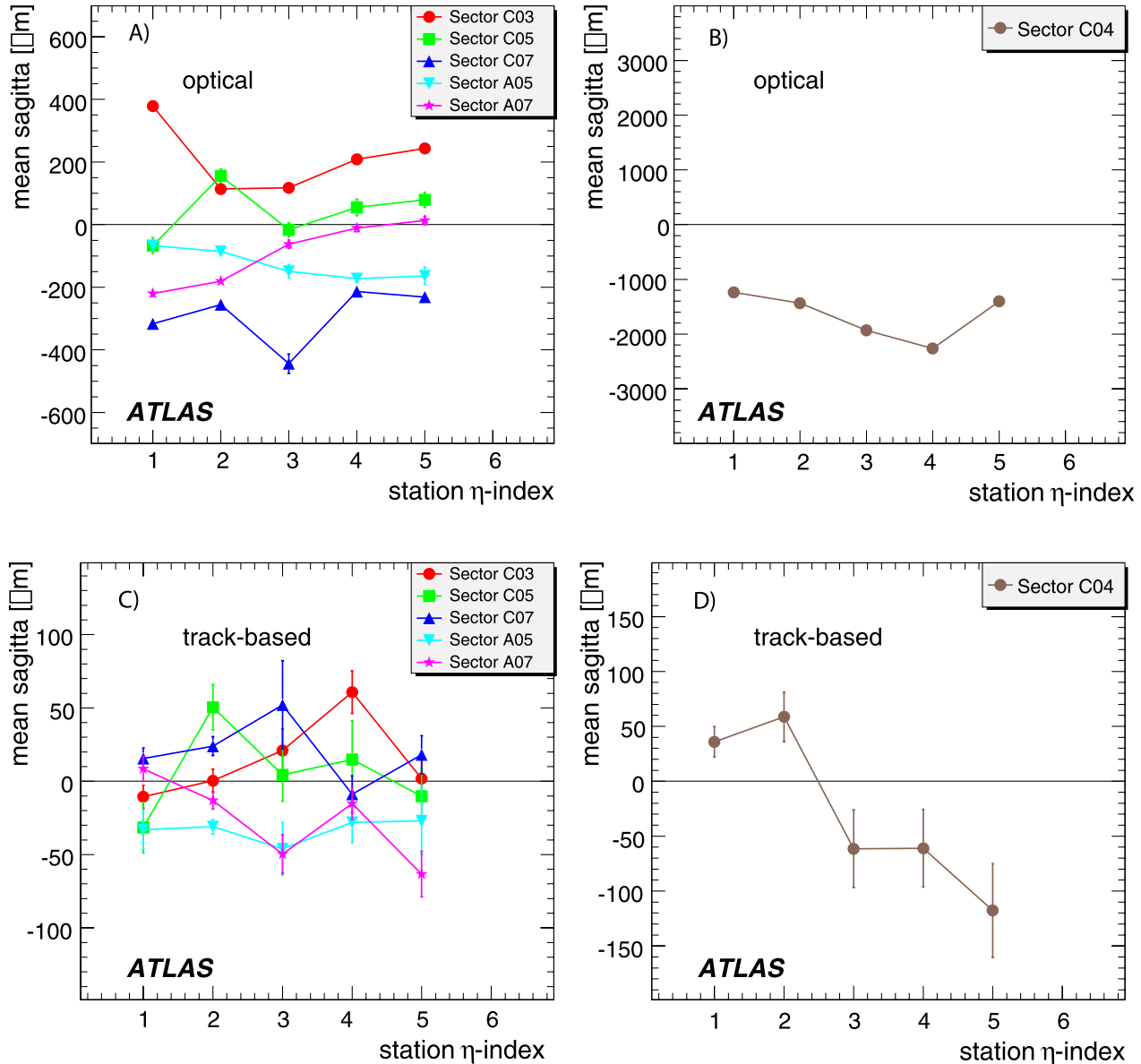

and Outer station) of MS projective towers. The RMS of the sagitta of the Middle station segment with respect to the Outer-Inner straight line extrapolation has been fit in five momentum bins. The result is shown in Fig. 28 for sector 5 (Large sector) with RPC-time corrections applied in the calibration procedure. The fitted value for the two terms is $K_{0}=(12.2 \pm 0.7) \mathrm{mm} \times \mathrm{GeV}$ and $K_{1}=(107 \pm 21) \mu \mathrm{m}$. In the MS the multiple scattering term is expected to be worse than the one measured at the test beam setup and larger for

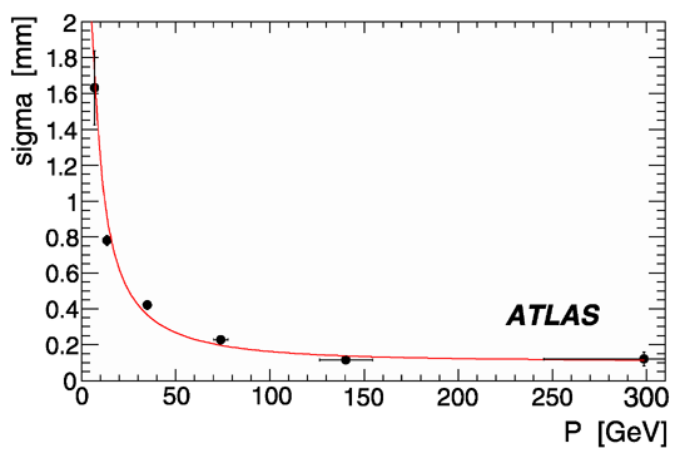

Fig. 28 RMS value of the sagitta distribution in sector 5 as a function of the muon momentum measured by the ID. The fit to the function described in the text is superimposed
Small sectors due to the presence of the toroid coils between the Inner and Outer chambers. The value of $K_{1}$ measured with cosmic muons in sector 5 is only about a factor two worse than that measured at the test beam. Several effects contribute to this, including alignment, chamber deformations, calibration and single tube resolution. Similar studies performed for other sectors show worse results due to the smaller data sample available for alignment and calibration.

These preliminary studies with cosmic rays indicate that the method of track-based alignment is robust and with sufficient muon data from collisions the design alignment precision will be achieved.

\section{Pattern recognition and segment reconstruction}

The pattern recognition algorithm first groups hits close in space and time for each detector. Each pattern is characterized by a position and a direction and contains all the associated hits. Starting from these patterns, the segments are reconstructed with a straight line fit. The $\mathrm{G} t_{0}$-refit is applied at this stage and, if the $\mathrm{G} t_{0}$-refit procedure does not converge, the segment parameters are computed with the tube 
$t_{0}$ provided by the calibration with tube resolution increased to $2 \mathrm{~mm}$. After this, a drift radius is assigned to each tube with an uncertainty of $2 \mathrm{~mm}$ (independent of the drift radius value) in order to keep high track reconstruction efficiency even in the case where no precise alignment constants are available. The minimum number of hits per segment was set to 3 and no cuts were applied on the number of missed hits.

These relaxed requirements tend to increase the number of fake segments while keeping a high segment efficiency. Since cosmic ray events are quite clean and have low hit multiplicity this fake rate increase is not considered as a problem. On the other hand, a high reconstruction efficiency allows the use of segments to spot hardware problems in individual chambers or in calibration or decoding software. Most of the fake segments are rejected at the track reconstruction level.

Figure 29(A) shows, on the left, the number of MDT hits per segment for segments associated to a track. In the distribution clear peaks are observed at 6 and 8 hits corresponding to the 6-layer (Middle and Outer) and 8-layer (Inner) chambers.

The efficiency of the segment reconstruction in run 91,060 was determined in the following way. First, cosmic shower events are suppressed by requiring less than 20 segments in the event. Then a pair of segments in two MDT stations (Inner, Medium or Outer) are fitted to a straight line and the line is extrapolated to the third station. In the extrapolation multiple scattering is taken into account assuming a $2 \mathrm{GeV}$ momentum for the cosmic muon. If the extrapolated line crossed the third station, a reconstructed segment is searched for in that station, but it is not required that the hits of this segment be associated to the muon track. The segment efficiency is then computed for each MDT chamber as the fraction of times a segment is found. In order to reduce the effect of the non-instrumented regions a fiducial cut in $\eta$ was applied for both barrel and end-cap. Chambers that were not operational in the analyzed run were removed from the sample. It was not possible to determine the efficiency for all chambers due to the limited coverage of the trigger for the run used for this analysis (Fall 2008) and flux of cosmic rays. For tracks crossing the overlap region between two adjacent chambers (Small/Large sector overlap) it was not required that two segments be reconstructed, since this may lead to a slight overestimation of the efficiency.

The distribution of the segment efficiency is shown in Fig. 29(B) for 322 chambers in the barrel. The average value is $99.5 \%$ and the segment efficiency is uniform over the acceptance as shown in Table 5. In the efficiency for the barrel chambers there is a small loss of about $0.5 \%$ due the presence of the support structure of the ATLAS barrel. The Inner chambers have a slightly lower segment efficiency due to the geometry of the trigger and a larger uncertainty in the track extrapolation. Studies on systematic effects in determining the segment efficiency, such as its dependence on the $\mathrm{G} t_{0^{-}}$
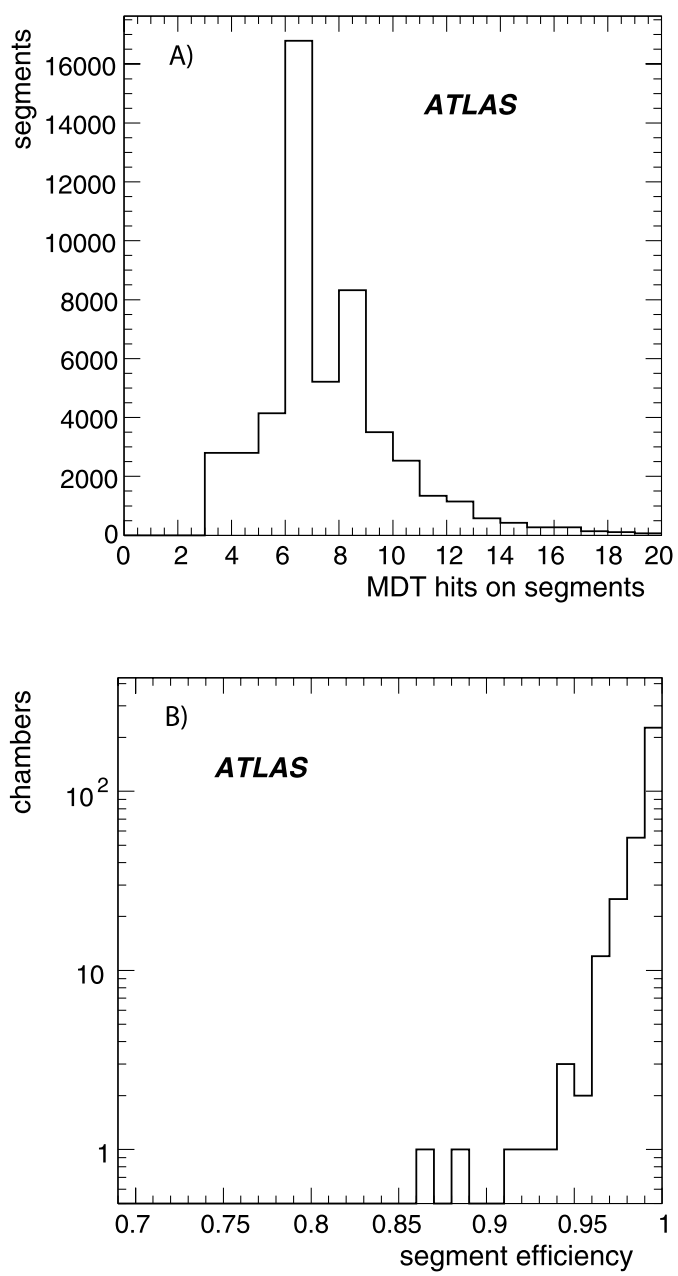

Fig. 29 (A): Distribution of the number of MDT hits per segment for segments associated to a track. (B): Segment reconstruction efficiency for 322 MDT chambers

Table 5 Average value of the segment reconstruction efficiency in the MDT stations

\begin{tabular}{lllllll}
\hline MDT station & BI & BM & BO & EI & EM & EO \\
\hline Segment efficiency & 0.987 & 0.992 & 0.996 & 0.992 & 0.998 & 0.999 \\
\hline
\end{tabular}

refit, on the extrapolation and on the track angle, show that a systematic error of $\sim 0.5 \%$ affects the values of efficiency listed in Table 5.

An alternative method to evaluate the segment reconstruction efficiency, almost independent of chamber hardware problems, is described in the following. As in the previous case, this method can be used only with no magnetic field in the MS. All segment pairs in two different MDT stations (Inner, Middle or Outer) with a polar angle difference smaller than $7.5 \mathrm{mrad}$ are considered. The segment pairs are fitted to a straight line and this is extrapolated to the third MDT station. The track is kept if at least three hit tubes are found in the third MDT with a signal charge above the ADC 
cut and aligned with the track extrapolation within one tube diameter, $\pm 3 \mathrm{~cm}$. The segment efficiency is then computed as the fraction of selected tracks that have a segment reconstructed with at least 3 hits in the identified drift tubes. Since the normalization already requires the presence of three hits in the tested MDT station, this segment efficiency is almost independent of local hardware problems. A segment reconstruction efficiency higher than 0.99 is found in all MDT stations.

The rate of fake segments was studied with a random trigger. An average rate of 0.06 fake segments per event was found with the relaxed hit association criteria used for cosmic muons. This rate is expected to be strongly reduced to about $2 \times 10^{-3}$ if the segment reconstruction requirements are made to be more stringent as shown by using an alternative muon tracking algorithm.

\section{Track reconstruction}

The MOORE and Muonboy programs have been optimized to reconstruct muon tracks originating from the IP. To cope with the different topology of cosmic ray muons they have been slightly modified as explained in Sect. 2. To mimic muons in collision events, the tracks are split at their perigee (point of closest approach to the beam axis), giving, usually, two reconstructed tracks: one in the upper part of the MS and one in the lower part. Events with at least one ID track satisfying the following criteria were selected:

- at least 20 hits in the Transition Radiation Tracker

- the number of hits summed over the SemiConductor

Tracker (SCT) and the Pixel detector greater than 4

- the distance of closest approach in the transverse plane

$\left|d_{0}\right|$ and along the $z$ axis $\left|z_{0}\right|$ smaller than $1 \mathrm{~m}$

- the value of the muon track $\chi^{2} /$ ndf $<3$

- the value of the reconstructed pseudorapidity $|\eta|<1$

- reconstructed momentum greater than $5 \mathrm{GeV}$.

This selection has been applied for all the studies reported in this section with the exception of the momentum resolution results.

\subsection{Resolution}

The distribution of residuals for MDT hits associated to a track is shown in Fig. 30. The hit residual is defined as the difference between the drift radius measured in a tube and the distance of the track to the tube wire. The distribution refers only to tracks with MDT hits in at least three different muon stations (Inner, Middle and Outer) because these tracks have well constrained parameters and individual hits give a small contribution to the track parameters. The distribution was fitted to a double Gaussian with common mean

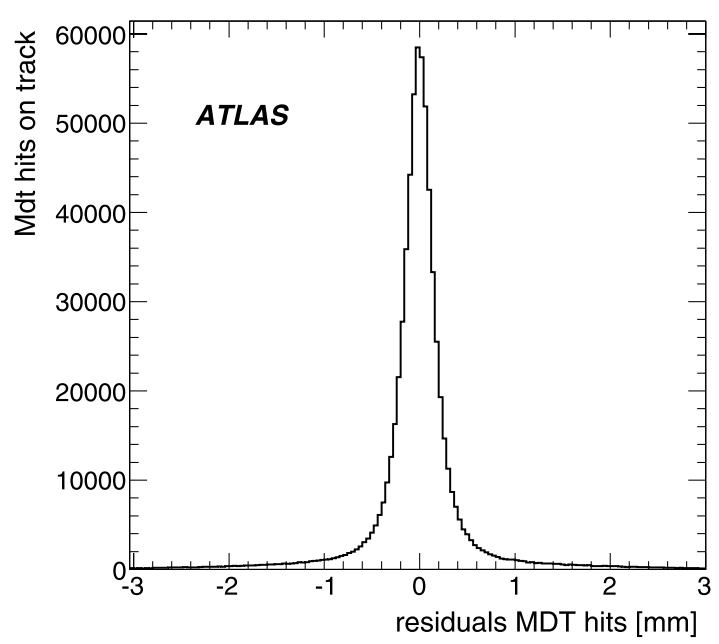

Fig. 30 Distribution of residuals for MDT hits associated to a track. The residuals have been fitted with a double-Gaussian function with common mean. The mean value is $6 \mu \mathrm{m}$, the standard deviation of the narrow Gaussian is about $150 \mu \mathrm{m}$ and the one of the wide Gaussian is about $700 \mu \mathrm{m}$

value. The mean of the distribution was $6 \mu \mathrm{m}$ and the RMS widths was $150 \mu \mathrm{m}$ for the narrow Gaussian, accounting for $75 \%$ of the distribution, and $700 \mu \mathrm{m}$ for the other. When compared to the distribution of the segment residuals shown in Sect. 6 two additional effects contribute to the broadening of this distribution: the misalignment between stations and multiple scattering in the MS material.

\subsection{Efficiency}

The track reconstruction efficiency is computed as the fraction of events where a track is reconstructed in the MS top or bottom hemisphere once an ID track was found satisfying the selection criteria described above. In this case also tracks with hits in only two out of three MDT stations (Inner, Middle or Outer) are accepted, even if these tracks have a worse momentum resolution than tracks reconstructed in three stations. About $15 \%$ of the selected tracks are in this category. In addition, to compute the track efficiency in the top (bottom) hemisphere, a momentum cut of $5 \mathrm{GeV}(9 \mathrm{GeV})$ on the ID track is applied. The result is shown in Fig. 31 as a function of the pseudorapidity of the ID track, for the top and bottom hemisphere separately.

The value of the efficiency, integrated over the $\eta$ acceptance, is $94.9 \%$ for the top and $93.7 \%$ for the bottom hemisphere respectively. If the four central bins are removed in Fig. 31 the efficiency increases to $98.3 \%$ and $96.3 \%$ respectively. The statistical error on these values is below $0.1 \%$. The lower efficiency in the central detector region, around $|\eta|=0$, is due to the presence of the main ATLAS service gap while lower efficiency in the Bottom hemisphere is explained by the uninstrumented regions occupied by the support structure of the ATLAS barrel. 


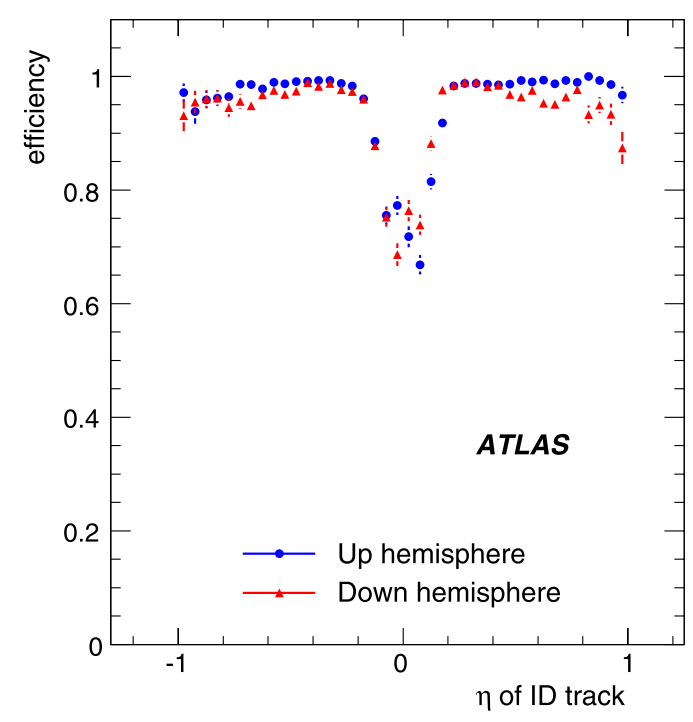

Fig. 31 Track reconstruction efficiency as a function of pseudorapidity. The loss in efficiency in the region near $|\eta|=0$ is due to the loss of acceptance for detector services. The presence of a track measured in the ID with $|\eta|<1$ is required

\subsection{Momentum measurement}

The momentum of cosmic muons was measured in runs with magnetic field. The momentum measurement can be defined at the MS entrance or at the point of closest approach to the IP. In the second case, for tracks crossing the ID, a correction was made for the energy loss in the calorimeters. This correction is based on the average energy loss computed by the track extrapolation algorithm and is on average $3.1 \mathrm{GeV}$ for muons pointing to the interaction region with a distance of closest approach of $\left|d_{0}\right|<1 \mathrm{~m}$ and $\left|z_{0}\right|<2 \mathrm{~m}$.

The distribution of momentum at the MS entrance is shown in Fig. 32-(A) for the top and bottom hemispheres separately. The difference between the two distributions is due to the ID track momentum cut of $5 \mathrm{GeV}$ that translates in a different momentum cut-off in the two MS hemispheres, since cosmic muons are directed downwards. The same distribution extrapolated to the perigee is shown in Fig. 32(B), demonstrating that the correction for the energy loss in the calorimeter removes the offset.

The distribution of the number of MDT hits associated with a track is shown in Fig. 33(A). For this plot tracks measured in three MDT stations have been selected. A clear peak around 20 hits is visible ( 8 tubes in the Inner stations, 6 in the Middle and Outer stations).

In events with tracks that cross the whole MS, the track is split at the perigee and the two independent momentum measurements, in the top and bottom hemisphere, can be compared. Figure 33(B) shows the distribution of the difference of the two momentum values, top-bottom measured at the MS entrance, for tracks with momenta greater than $15 \mathrm{GeV}$. In this case the muons cross the calorimeter twice
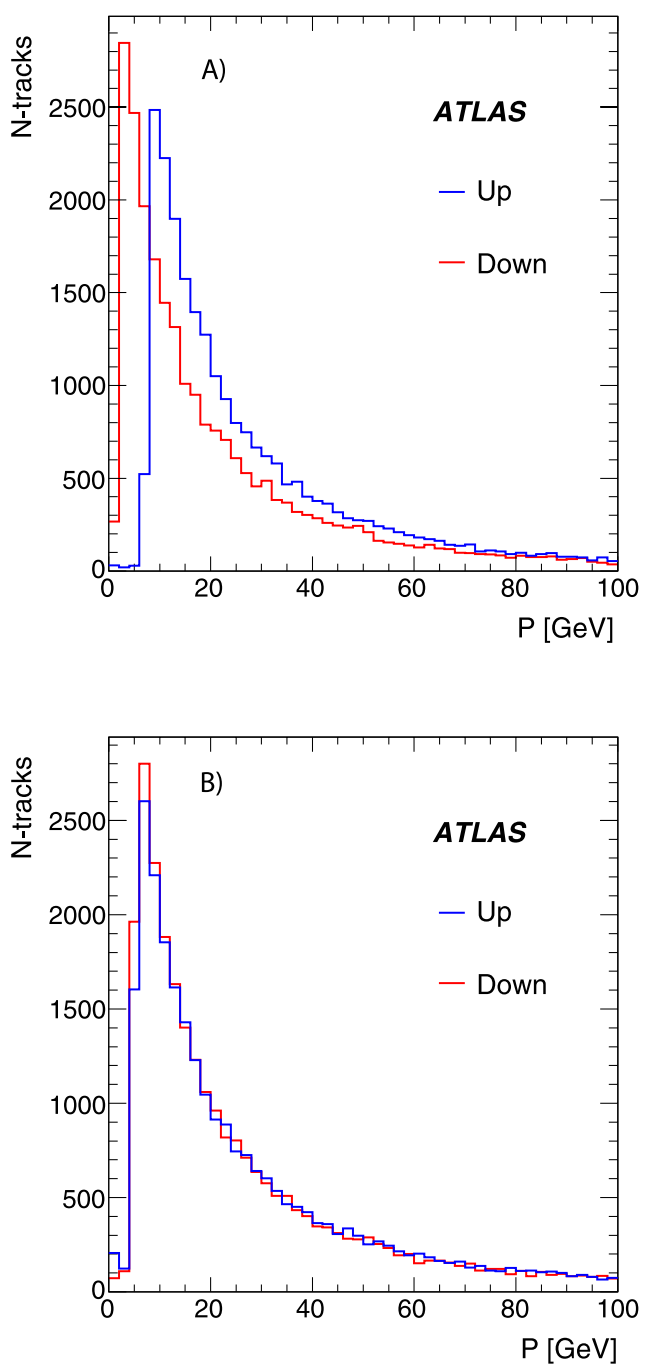

Fig. 32 (A): Distribution of momentum of cosmic muons as measured at the MS entrance for the upper and lower hemispheres. The difference between the two distributions is due to the ID track momentum cut of $5 \mathrm{GeV}$. (B): Same distributions with track momentum extrapolated to the IP

and the energy loss is twice the value quoted above, in good agreement with the $6.3 \mathrm{GeV}$ mean value of the distribution.

The MS momentum resolution has been estimated by comparing for each cosmic muon the two independent measurements in the top and bottom hemispheres. In order to increase the available statistics no requirements on the presence of ID tracks were applied in this study. Only events with at least two reconstructed tracks in the MS are considered. Each track is required to have:

- at least 17 MDT hits, of which at least 7 in the Inner and 5 in the Middle and Outer stations of the same $\phi$ sector

- at least 2 different layers of RPC with a hit in the $\phi$ projection

- polar angle $65^{\circ}<\theta<115^{\circ}$ 
- distance of closest approach to the IP $\left|d_{0}\right|<1 \mathrm{~m}$ and $\left|z_{0}\right|<2 \mathrm{~m}$

- polar and azimuthal angles of the MS track pair agree within $10^{\circ}$.

About $19 \mathrm{~K}$ top-bottom track pairs were selected in this way. For each track the value of transverse momentum was evaluated at the IP. The difference between the two values divided by their average

$\frac{\Delta p_{T}}{p_{T}}=2 \frac{p_{\text {Tup }}-p_{\text {Tdown }}}{p_{\text {Tup }}+p_{\text {Tdown }}}$

was measured in eleven bins of $p_{T}$. Since the cosmic muon momentum distribution is a steep function (see Fig. 32), the $p_{T}$ value of each bin was taken as the mean value of the distribution in that bin.
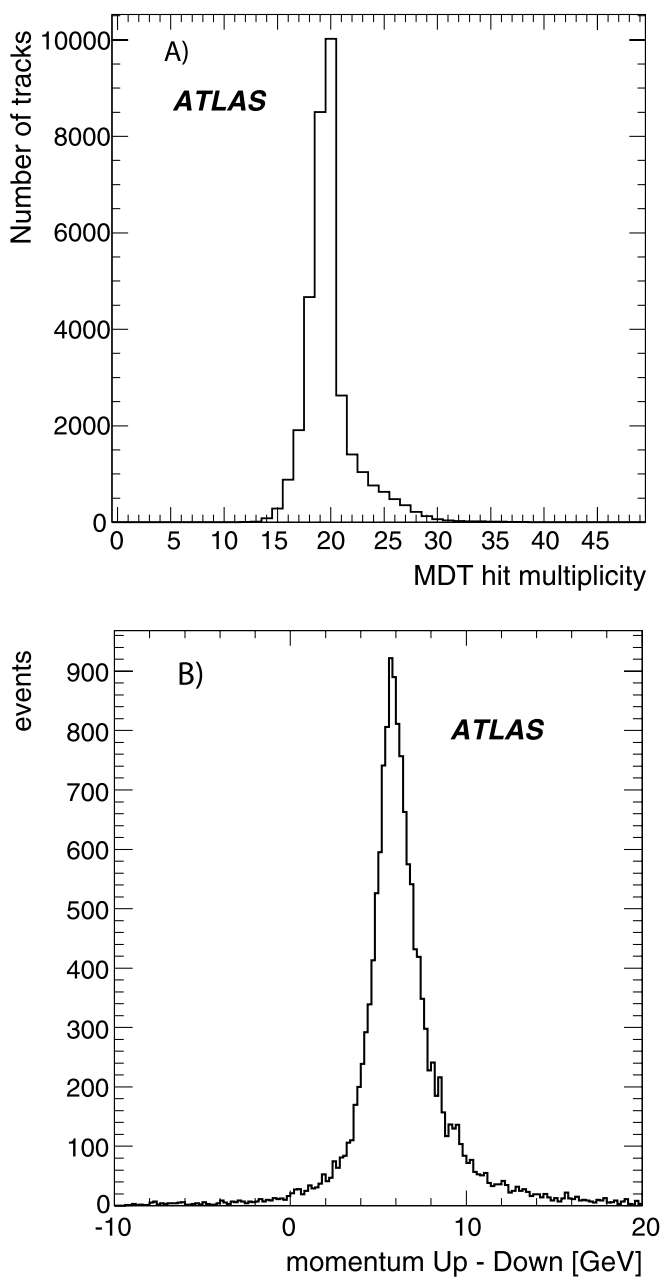

Fig. 33 (A): Number of MDT hits on track. (B): Momentum difference between momenta measured by the MS in the top and bottom hemispheres for cosmic muons. The momenta are expressed at the MS entrance and only tracks with momenta bigger than $15 \mathrm{GeV}$ are considered. The mean value of $6.3 \mathrm{GeV}$ is due to the energy loss in the calorimeter material
The distribution of $\Delta p_{T} / p_{T}$ was fitted in each bin with a double-Gaussian function with common mean value. The narrow Gaussian was convoluted with a Landau function to account for the distribution of energy loss in the calorimeter. For $p_{T}<10 \mathrm{GeV}$ the normalizations of the two Gaussians were constrained such that $95 \%$ of the events are in the narrow Gaussian. Above $10 \mathrm{GeV}$ this constraint was lowered to $70 \%$. The mean value is representative of the difference in the transverse momentum scale between the two MS hemispheres. The RMS of the narrow Gaussian plus the width of the Landau, divided by $\sqrt{2}$, is taken as an estimate of the transverse momentum resolution for each $p_{T}$ bin. The Landau width is added linearly to the narrow Gaussian RMS since the two quantities are strongly correlated.

The distribution of $\Delta p_{T} / p_{T}$ is shown in Fig. 34 for all $p_{T}$ bins together with the fitted function. For the eleven bins the fit probability is in the range between $45 \%$ and $99 \%$, showing that the chosen parametrization is a good representation of the data distribution.

Different fits have been done to study the systematics of the mean and RMS value. (i) The constraint between the two Gaussian areas has been changed by $\pm 10 \%$. (ii) A double Gaussian with common mean and asymmetric fit range, with the fit range reduced to two standard deviations on the positive side to avoid the energy loss tail. (iii) A fit with two independent Gaussians with no range constraint. The result is that the estimated resolution is quite independent of the fit assumptions. The variation of the fit resolution ranges between $0.5 \%$ at low $p_{T}$ up to a maximum of $1 \%$ in the highest momentum bin.

The fit mean values indicate that the $p_{T}$ scales in the two MS hemispheres are in agreement within $1 \%$, or better. The relative $p_{T}$ resolution, $\sigma_{p_{T}} / p_{T}=\sigma\left(\Delta p_{T} / p_{T}\right) / \sqrt{2}$, is shown in Fig. 35, for the two main muon reconstruction algorithms [7, 8], as a function of the transverse momentum. The two results are consistent taking into account the independent statistical uncertainties.

The resolution function can be fitted with the sum in quadrature of three terms, the uncertainty on the energy loss corrections $P_{0}$, the multiple scattering term $P_{1}$, and the intrinsic resolution $P_{2}$.

$\frac{\sigma_{p_{T}}}{p_{T}}=\frac{P_{0}}{p_{T}} \oplus P_{1} \oplus P_{2} \times p_{T}$.

The result of the fit is shown in Fig. 35. The values of the parameters are: $P_{0}=0.29 \pm 0.03 \pm 0.01 \mathrm{GeV}, P_{1}=0.043 \pm$ $0.002 \pm 0.002, P_{2}=(4.1 \pm 0.4 \pm 0.6) \times 10^{-4} \mathrm{GeV}^{-1}$. The second uncertainty, due the systematics of the bin-by-bin fit method, was evaluated by changing the fitting assumptions as explained above. The expected values for these parameters were computed in reference [3] on the basis of an analytic calculation of the $p_{T}$ resolution that takes into account the detailed description of the material in the MS, the 

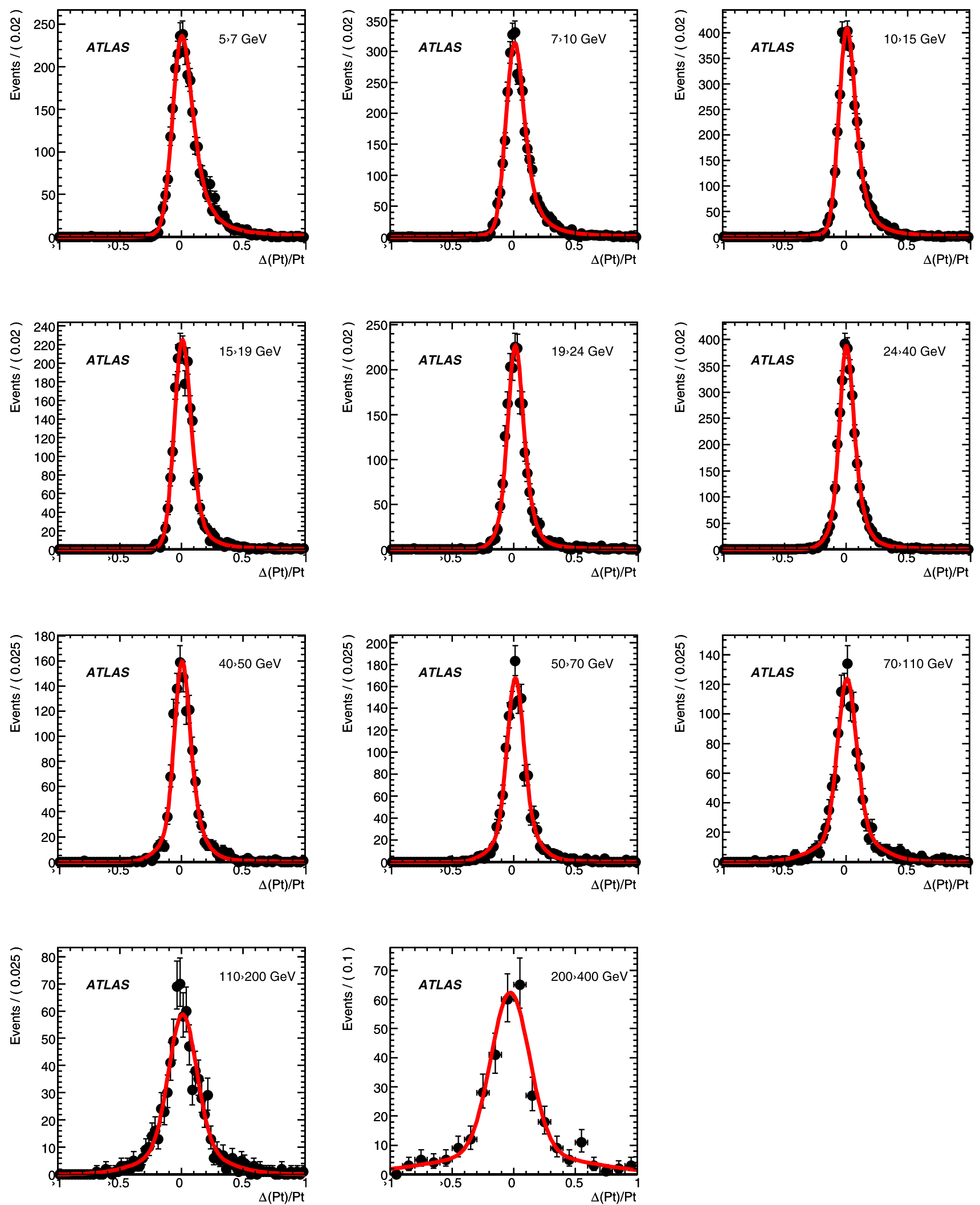

Fig. 34 Distributions of $\Delta p_{T} / p_{T}$ in the eleven $p_{T}$ bins. Fits to the function described in the text are superimposed 
Fig. 35 Transverse momentum resolution evaluated with the top-bottom method explained in the text as a function of $p_{T}$, barrel region only $(|\eta|<1.1)$. The fit to the three resolution parameters as described in the text is superimposed

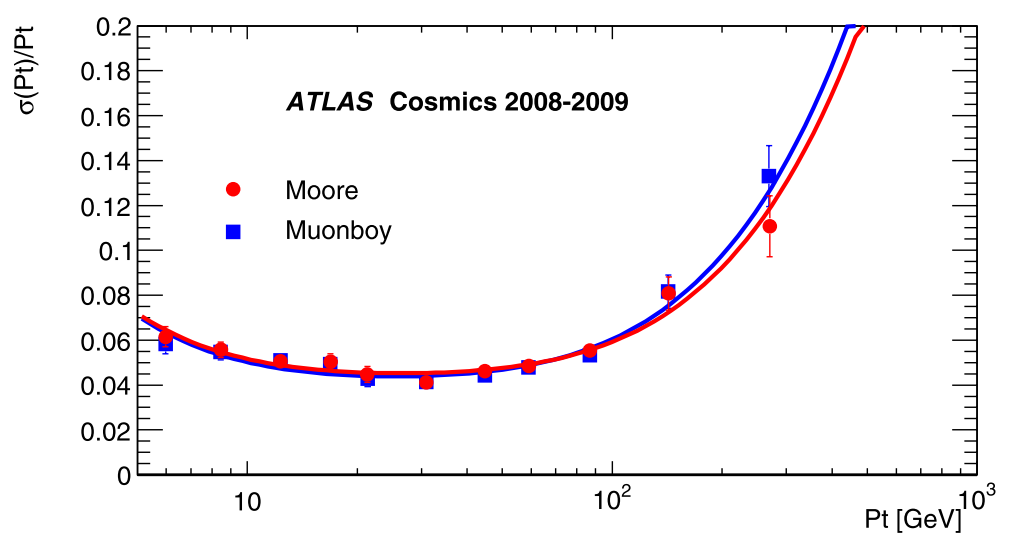

single tube resolution, the alignment accuracy and the magnetic field map. The values obtained for the barrel MS were: $P_{0}=0.35 \mathrm{GeV}, P_{1}=0.035$ and $P_{2}=1.2 \times 10^{-4} \mathrm{GeV}^{-1}$. The result is in fair agreement with the expected values for the first two terms, while the intrinsic term is worse. The difference has been investigated to trace the effects that contribute to worsen the resolution as determined with cosmic muons.

First, more than $70 \%$ of the track pairs considered in the analysis are in the Large sectors 5-13. At high $p_{T}$ the momentum resolution in the barrel Large sectors is worse than in Small sectors because the field integral is smaller (see Fig. 1). Instead, in the low $p_{T}$ region dominated by multiple scattering the resolution in Large sectors is better since the magnet coils are in the Small sectors.

Second, the single tube resolution is affected by imperfect calibrations and the additional time jitter is not completely recovered by the $\mathrm{G} t_{0}$-refit (see Fig. 12). Part of the tracks in the sample contain segments with a badly converging $\mathrm{G} t_{0}$-refit. As a cross-check, all the tracks with bad convergence were removed and the analysis was repeated. The intrinsic term decreased by about $30 \%$.

Third, the alignment in many sectors of the MS is still not at the required level due to the limited statistics of straight tracks in the cosmic ray data sample. Last, several other effects that contribute to resolution have not been removed, such as chamber deformations (due to temperature effects), wire sagging (particularly important in large chambers), single chamber geometrical defects. Each of these effects contribute to worsening the resolution and can be removed with dedicated software tools. At the present stage of commissioning, the momentum resolution is close to the design value for $p_{T}<50 \mathrm{GeV}$, but is not as good for higher momenta.

\section{Summary}

The data collected in several months during the 2008-2009 cosmic ray runs have been analyzed to assess the perfor- mance of the Muon Spectrometer after its installation in the ATLAS experiment. Parts of the detector, the Small Wheels in front of the end-cap toroids, were installed during the runs and the commissioning of the many detectors was proceeding while debugging the data acquisition and the data control systems. The detector coverage during most of the run period was higher than $99 \%$, with the exception of the RPC chambers which were still under commissioning. For this detector subsystem the coverage steadily improved during the commissioning runs reaching more than $95 \%$ in Spring 2009. Results on several aspects of the Muon Spectrometer performance have been presented. These include detector coverage, efficiency, resolution and relative timing of trigger and precision tracking chambers, track reconstruction, calibration, alignment and data quality.

Finally, with data collected when the magnetic field was on, a first estimate of the spectrometer momentum resolution was obtained. Efficiency and resolution of single elements have been measured for MDT, RPC and TGC chambers and were found in agreement with results obtained previously with high-momentum muon beams. The trigger chamber timing has been adjusted with enough precision to guarantee that the interaction bunch crossing can be identified with a minimal number of failures. The muon trigger logic, based on fast tracking of pointing muons has been extensively tested in the regions of the detector with good cosmic ray illumination. A slight deterioration of the MDT spatial resolution, compared to test beam results, was observed, which can be understood in terms of an additional time jitter due to the asynchronous timing of cosmic muons and to their non-pointing geometry. These effects were partially removed, modifying the track reconstruction programs with dedicated algorithms. Allowing for an increase of the single hit resolution, to cope with these effects, the track segment efficiency in individual chambers was found to be satisfactory and uniform over the large number of chambers.

The performance of the end-cap and barrel optical alignment systems have been measured using cosmic muon tracks with no magnetic field. The results demonstrate that 
the end-cap optical system is able to provide the required precision for chamber alignment. The design of the alignment system in the barrel requires additional constraints provided by straight tracks. The method has been tested with good results, but is limited by the statistics of highmomentum muons with the required pointing geometry.

With the geometry corrections provided by the alignment system, tracks in projective geometry were reconstructed in the barrel showing that the reconstruction efficiency is uniform over the entire acceptance and that the sagitta error is in agreement with the detector resolution, the alignment precision and the effect of multiple coulomb scattering.

Finally with magnetic field, tracks crossing the whole spectrometer were used to obtain two independent measurements of the momentum. The momentum resolution was evaluated using the two values in the top and bottom part of the detector and the results were analyzed, fitting the distribution of the difference as function of the momentum. Taking into account the momentum spectrum, the multiple scattering in the spectrometer and the energy loss in traversing the calorimeters, the momentum resolution is in good agreement with results from simulation for transverse momenta smaller than $50 \mathrm{GeV}$. The statistics of highmomentum pointing tracks limits the accuracy of the individual chamber calibration and the precision of the alignment. At higher momenta, these limitations result in degraded momentum resolution.

During the long period of commissioning with cosmic rays it was possible to optimize the performance of the various hardware and software elements and to reach a level of understanding, such that we can consider the Muon Spectrometer to be ready to efficiently detect muons produced in high-energy proton-proton collisions.

Acknowledgements We are greatly indebted to all CERN's departments and to the LHC project for their immense efforts not only in building the LHC, but also for their direct contributions to the construction and installation of the ATLAS detector and its infrastructure. We acknowledge equally warmly all our technical colleagues in the collaborating Institutions without whom the ATLAS detector could not have been built. Furthermore we are grateful to all the funding agencies which supported generously the construction and the commissioning of the ATLAS detector and also provided the computing infrastructure.

The ATLAS detector design and construction has taken about fifteen years, and our thoughts are with all our colleagues who sadly could not see its final realization.

We acknowledge the support of ANPCyT, Argentina; Yerevan Physics Institute, Armenia; ARC and DEST, Australia; Bundesministerium für Wissenschaft und Forschung, Austria; National Academy of Sciences of Azerbaijan; State Committee on Science \& Technologies of the Republic of Belarus; CNPq and FINEP, Brazil; NSERC, NRC, and CFI, Canada; CERN; CONICYT, Chile; NSFC, China; COLCIENCIAS, Colombia; Ministry of Education, Youth and Sports of the Czech Republic, Ministry of Industry and Trade of the Czech Republic, and Committee for Collaboration of the Czech Republic with CERN; Danish Natural Science Research Council and the Lundbeck Foundation; European Commission, through the ARTEMIS Research Training Network; IN2P3-CNRS and Dapnia-CEA, France; Georgian Academy of Sciences; BMBF, HGF, DFG and MPG, Germany; Ministry of
Education and Religion, through the EPEAEK program PYTHAGORAS II and GSRT, Greece; ISF, MINERVA, GIF, DIP, and Benoziyo Center, Israel; INFN, Italy; MEXT, Japan; CNRST, Morocco; FOM and NWO, Netherlands; The Research Council of Norway; Ministry of Science and Higher Education, Poland; GRICES and FCT, Portugal; Ministry of Education and Research, Romania; Ministry of Education and Science of the Russian Federation and State Atomic Energy Corporation "Rosatom"; JINR; Ministry of Science, Serbia; Department of International Science and Technology Cooperation, Ministry of Education of the Slovak Republic; Slovenian Research Agency, Ministry of Higher Education, Science and Technology, Slovenia; Ministerio de Educación y Ciencia, Spain; The Swedish Research Council, The Knut and Alice Wallenberg Foundation, Sweden; State Secretariat for Education and Science, Swiss National Science Foundation, and Cantons of Bern and Geneva, Switzerland; National Science Council, Taiwan; TAEK, Turkey; The Science and Technology Facilities Council and The Leverhulme Trust, United Kingdom; DOE and NSF, United States of America.

Open Access This article is distributed under the terms of the Creative Commons Attribution Noncommercial License which permits any noncommercial use, distribution, and reproduction in any medium, provided the original author(s) and source are credited.

\section{References}

1. The ATLAS Muon Collaboration, The ATLAS Muon Spectrometer technical design report. CERN-LHCC/97-22 (31 May 1997). ISBN 92-9083-108-1

2. G. Aad et al. (The ATLAS Collaboration), The ATLAS experiment at the CERN large hadron collider. J. Instrum. 3, S08003 (2008). $1-437$

3. G. Aad et al. (The ATLAS Collaboration), Expected Performance of the ATLAS Experiment: Detector, Trigger and Physics. CERNOPEN 2008-020 (December 2008). ISBN 978-92-9083-321-5

4. C. Adorisio et al., System Test of the ATLAS Muon spectrometer in the $\mathrm{H} 8$ beam at the CERN SPS. Nucl. Instrum. Meth. A 593, 232-254 (2008)

5. C. Adorisio et al., Study of the ATLAS MDT spectrometer using high energy CERN combined test beam data. Nucl. Instrum. Meth. A 598, 400-415 (2009)

6. The ATLAS Collaboration, ATLAS computing technical design report. CERN-LHCC/2005-022 (20 June 2005). ISBN 92-9083250-9

7. D. Adams et al., Track reconstruction in the ATLAS Muon spectrometer with MOORE. ATL-SOFT-2003-007, 2.10.2003, http://cdsweb.cern.ch/collection/ATLAS

8. S. Hassani et al., A Muon identification and combined reconstruction procedure for the ATLAS detector at the LHC using (Muonboy, STACO, MuTag) reconstruction packages. Nucl. Instrum. Meth. A 572, 77-79 (2007)

9. The ATLAS Collaboration, First level trigger technical design report. CERN-LHCC/98-014 (30 June 1998). ISBN 92-9083-128-6

10. F. Anulli et al., The level-1 Muon barrel trigger of the ATLAS experiment. J. Instrum. 4, P04010 (2009). 1-35

11. The ATLAS Collaboration, High-level trigger, data acquisition and controls technical design report. CERN-LHCC/2003-022 (30 June 2003). ISBN 92-9083-205-3

12. P. Bagnaia et al., Calibration model for the MDT chambers of the ATLAS Muon spectrometer. ATL-MUON-PUB-2008-004 (28 March 2008), http://cdsweb.cern.ch/collection/ATLAS

13. M. Verducci, ATLAS database experience with the COOL conditions database project. J. Phys. Conf. Ser. 119, 042031 (2008) 
14. Y. Arai, Development of front end electronics and TDC LSI for the ATLAS MDT. Nucl. Instrum. Meth. A 453, 365 (2000)

15. Y. Arai et al., ATLAS muon drift tube electronics. J. Instrum. 3, P09001 (2008). 1-58

16. D.S. Levin et al., Drift time spectrum and gas monitoring in the ATLAS Muon spectrometer precision chambers. Nucl. Instrum. Meth. A 588, 347-358 (2008)

17. R. Veenhof, Garfield, a drift chamber simulation program. Prepared for International Conference on Programming and Mathematical Methods for Solving Physical Problems, Dubna, Russia, 14-19 June 1993

18. S.F. Biagi, Monte Carlo simulation of electron drift and diffusion in counting gases under the influence of electric and magnetic fields. Nuclear Instrum. Methods Phys. Res., Sect. A, Accel. Spectrom. Detect. Assoc. Equip. 421(1-2), 234-240 (1999)
19. R.M. Avramidou, E. Gazis, R. Veenhof, Drift properties of the ATLAS MDT chambers. Nucl. Instrum. Meth. A 568, 672-681 (2006)

20. P. Branchini et al., Global time fit for tracking in an array of drift cells: the drift tubes of the ATLAS experiment. IEEE Trans. Nucl. Sci. 55, 620 (2008)

21. C. Amelung, The alignment system of the ATLAS Muon spectrometer. Eur. Phys. J. C 33, 999-1001 (2004)

22. S. Aefsky et al., The optical alignment system of the ATLAS Muon spectrometer endcaps. J. Instrum. 3, P11005 (2008). pp. $1-59$

23. C. Guyot et al., The alignment system of the barrel part of the ATLAS Muon spectrometer. ATLAS Note ATL-MUON-PUB-2008007 (2008), http://cdsweb.cern.ch/collection/ATLAS

24. V. Blobel, Millepede: linear least squares fits with a large number of parameters, http://www.desy.de/ blobel/mptalks.html 